\title{
PANTEX PLANT SITE ENVIRONMENTAL REPORT FOR CALENDAR YEAR 1988
}

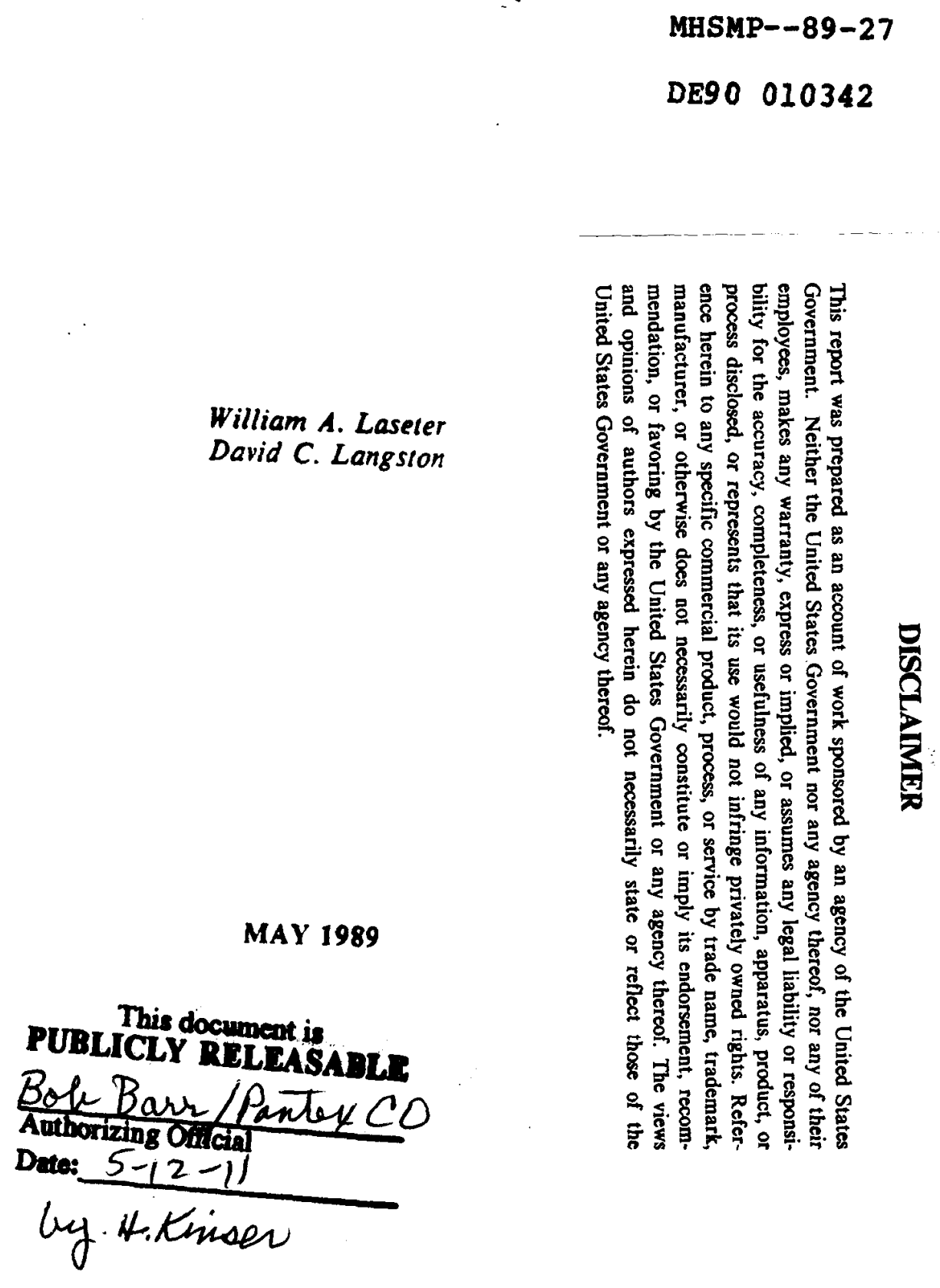

Environment, Safety, and Health Division 


\section{DISCLAIMER}

This report was prepared as an account of work sponsored by an agency of the United States Government. Neither the United States Government nor any agency Thereof, nor any of their employees, makes any warranty, express or implied, or assumes any legal liability or responsibility for the accuracy, completeness, or usefulness of any information, apparatus, product, or process disclosed, or represents that its use would not infringe privately owned rights. Reference herein to any specific commercial product, process, or service by trade name, trademark, manufacturer, or otherwise does not necessarily constitute or imply its endorsement, recommendation, or favoring by the United States Government or any agency thereof. The views and opinions of authors expressed herein do not necessarily state or reflect those of the United States Government or any agency thereof. 


\section{DISCLAIMER}

Portions of this document may be illegible in electronic image products. Images are produced from the best available original document. 


\section{CONTENTS}

Page

ABSTRACT . . . . . . . . . . . . . . . . . . . . . . . . 1

INTRODUCTION . . . . . . . . . . . . . . . . . . . . . . . . . . . . 2

ENVIRONMENTAL SETTING . . . . . . . . . . . . . . . 5

ENVIRONMENTAL ACTIVITIES . . . . . . . . . . . . . . . 7

ENVIRONMENTAL PROGRAM . . . . . . . . . . . . . . . . . . . . . . . . . . 8

AIR . . . . . . . . . . . . . . . . . . . . . . . . 12

Radioactive... . . . . . . . . . . . . . . . . 12

Nonradioactive . . . . . . . . . . . . . . . . . . 13

WATER . . . . . . . . . . . . . . . . . . . . . . 13

Radioactive . . . . . . . . . . . . . . . . . . . 13

Nonradioactive . . . . . . . . . . . . . . . . . . . . 14

SOIL . . . . . . . . . . . . . . . . . . . . . . 15

Radioactive... . . . . . . . . . . . . . . . 15

Nonradioactive . . . . . . . . . . . . . . . . . . 15

VEGETATION . . . . . . . . . . . . . . . . . . . . . . . . . 15

Radioactive... . . . . . . . . . . . . . . . . 15

BIOTA . . . . . . . . . . . . . . . . . . . . . . . 15

Radioactive... . . . . . . . . . . . . . . . 15

CALCULATION OF POTENTIAL RADIATION DOSE TO THE PUBLIC

FROM PANTEX ACTIVITIES FOR 1988 . . . . . . . . . . . . . . . . . . . . . 16

SUMMARY . . . . . . . . . . . . . . . . . . . . . . . 17

REFERENCES . . . . . . . . . . . . . . . . . . . . . . . . . 92

APPENDIX A . . . . . . . . . . . . . . . . . . . . . . . . . A-I

DISTRIBUTION . . . . . . . . . . . . . . . . . . . . . . B-1 


\section{FIGURES}

Figure

Page

1 Pantex Plant Vicinity Map ................

2

2 Pantex Plant Facility Site . . . . . . . . . . . . .

3

3 Physical Features of the Texas High Plains . . . . . . . .

5

4 Annual Amarillo Wind Rose for 1987 . . . . . . . . . . 6

5 Off-Site Air Sampling Locations . . . . . . . . . . . . 8

6 Plant Site Air Sampling Locations . . . . . . . . . . . . .

Off-Site Environmental Soil and Vegetation Sampling Locations for Pantex Plant [8-kilometer (5-mile) Radius] . . . . . . 10

$8 \quad$ Plant Site Soil and Vegetation Sampling Locations.......

9 Burning Ground Soil and Vegetation Sampling Locations.... .

10 Firing Site Soil and Vegetation Sampling Locations . . . . .

11 Plant Site Water Sampling Locations.............

12 Population Within an 80-kilometer (50-mile) Radius of.... 


\section{TABLES}

Tables

Page

I Estimated Atmospheric Releases of Radioactive Material from Pantex Plant for 1988

Estimated Steam Generating Plant Emissions for 1988

Vicinity Dose Calculations from Pantex Plant Contributions for 1988

IV Pantex Vicinity Population Dose Due to Naturally Occurring Penetrating Radiation.............. 18

Summary of Tritium in Air for 1988

Summary of Plutonium-239 in Air for 1988

VII Summary of Uranium-234 in Air for 1988 . . . . . . . . . . . . 21

VIII Summary of Uranium-238 in Air for 1988 . . . . . . . . . . . . 22

IX Summary of Tritium in Water for 1988 . . . . . . . . . . . . . 23

$\mathrm{X}$ Summary of Plutonium-239 in Water for 1988 . . . . . . . . . . 24

XI Summary of Uranium-234 in Water for 1988 . . . . . . . . . . . 25

XII Summary of Uranium-238 in Water for 1988 . . . . . . . . . . . 26

XIII Summary of Radium-226 in Water for 1988 . . . . . . . . . . . . 27

XIV Summary of Radium-228 in Water for 1988 . . . . . . . . . . . . 28

XV Summary of Dissolved Alpha in Water for 1988 . . . . . . . . . 29

XVI Summary of Suspended Alpha in Water for 1988 . . . . . . . . . 30

XVII Summary of Dissolved Beta in Water for 1988 . . . . . . . . . . 31

XVIII Summary of Suspended Beta in Water for 1988 . . . . . . . . . . 32

XIX Summary of Beryllium in Soll for 1988 . . . . . . . . . . . . 33

XX Summary of Plutonium-239 in Soll for 1988 . . . . . . . . . . . 34

XXI Summary of Uranium-234 in Soll for 1988 . . . . . . . . . . . 36

XXII Summary of Uranium-238 in Soil for 1988 . . . . . . . . . . . 38

XXIII Summary of Tritium in Vegetation for 1988 . . . . . . . . . . . 40

XXIV Summary of Uranium-234 in Vegetation for 1988 . . . . . . . . . 41

XXV Summary of Uranium-238 in Vegetation for 1988 . . . . . . . . . 42

XXVI Summary of Fluoride In Vegetation for 1988 . . . . . . . . . . 43

XXVII Summary of Tritium in Jackrabbits for 1988 . . . . . . . . . . 43

XXVIII Summary of Plutonium-239 in Jackrabbits for 1988 . . . . . . . 44

XXIX Summary of Uranium-234 in Jackrabbits for 1988 . . . . . . . . 44

XXX Summary of Uranium-238 in Jackrabbit for 1988 . . . . . . . . . 45 


\section{TABLES (Cont'd)}

Tables

Page

XXXI Summary of Organ Weights of Jackrabbits for 1988 . . . . . . . 45

XXXII Summary of Molsture of Jackrabbits Organs for 1988 . . . . . 46

XXXIII Summary of Ash of Jackrabbits Organs for 1988 . . . . . . . . 46

XXXIV Summary of Chemical Analysis for Water Pollutants for 1988 . . 47 
$$
\text { . }
$$ 


\title{
PANTEX PLANT SITE ENVIRONMENTAL REPORT \\ FOR CALENDAR YEAR 1988
}

William A. Laseter

David C. Langston

MAY 1989

Environment, Safety and Health Division

\begin{abstract}
This report summarizes the environmental monitoring program at Pantex Plant for 1988. It has been prepared in accordance with the United States Department of Energy Order 5484.1. This report presents monitoring data for both radioactive and nonradioactive species in the local environment.

Plant activities involve the handling of significant quantities of uranium, plutonium and tritium in the form of completed parts received from other DOE facilities, resulting in a very low potential for release of these radionuclides to the atmosphere. In 1988 only small releases of tritium occurred which could have affected the local environment. Monitoring data indicate that concentrations of this nuclide in the environment are below established criteria for air and water and therefore should not present a health hazard either to employees or to the public.
\end{abstract}




\section{INTRODUCTION}

Pantex Plant is a government-owned contractor-operated facility owned by the United States Department of Energy (DOE) and administered through the Amarillo Area Office (AAO). Mason \& Hanger - Silas Mason Co., Inc., is the prime operating contractor.

Pantex Plant is located in the Panhandle of Texas in Carson County. The facility is approximately 27 kilometers $(17$ miles) northeast of downtown Amarillo, and 16 kilometers (10 miles) west of downtown Panhandle, at $101^{\circ} 33^{\prime}$ West longitude and $35^{\circ}$ 19' North latitude (Figure 1). The plant site consists of 5,412 hectares (13,367 acres) which is a portion of the former 6,478 hectare (16,000-acre) Pantex Army Ordnance Plant which was constructed in 1942 for World War II conventional shell and bomb loading.

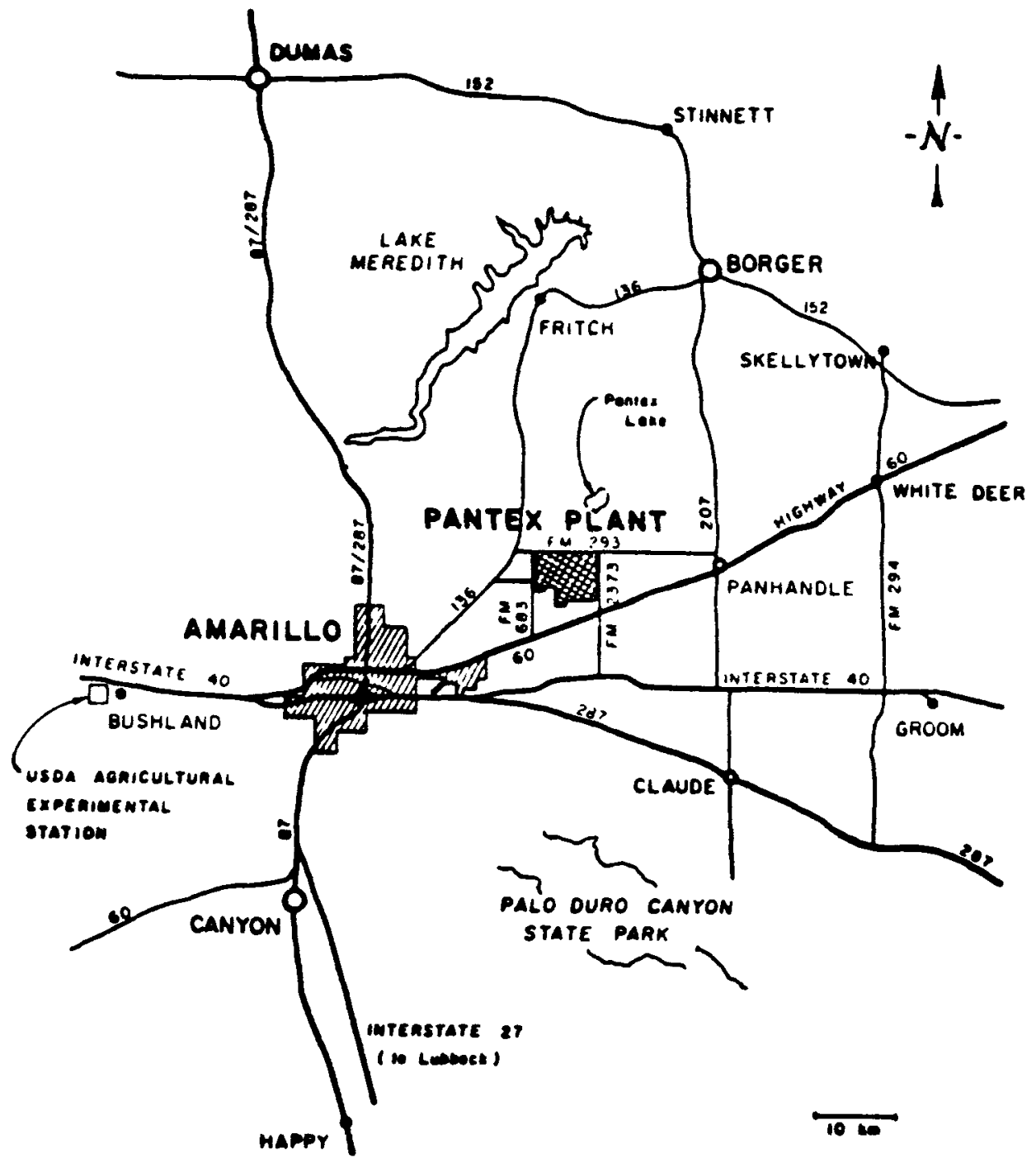

Figure 1. Pantex Fiant Vicinity Map 
Operations of the Pantex Plant take place in several widely separated zones (Figure 2). The operating zones (and the land surrounding them required for afety buffer or security zones) utilize approximately $30 \%$ of the plant's cotal area. The remaining $70 \%$ is utilized for agricultural research purpases through an agreement between Texas Tech University and the DOE.

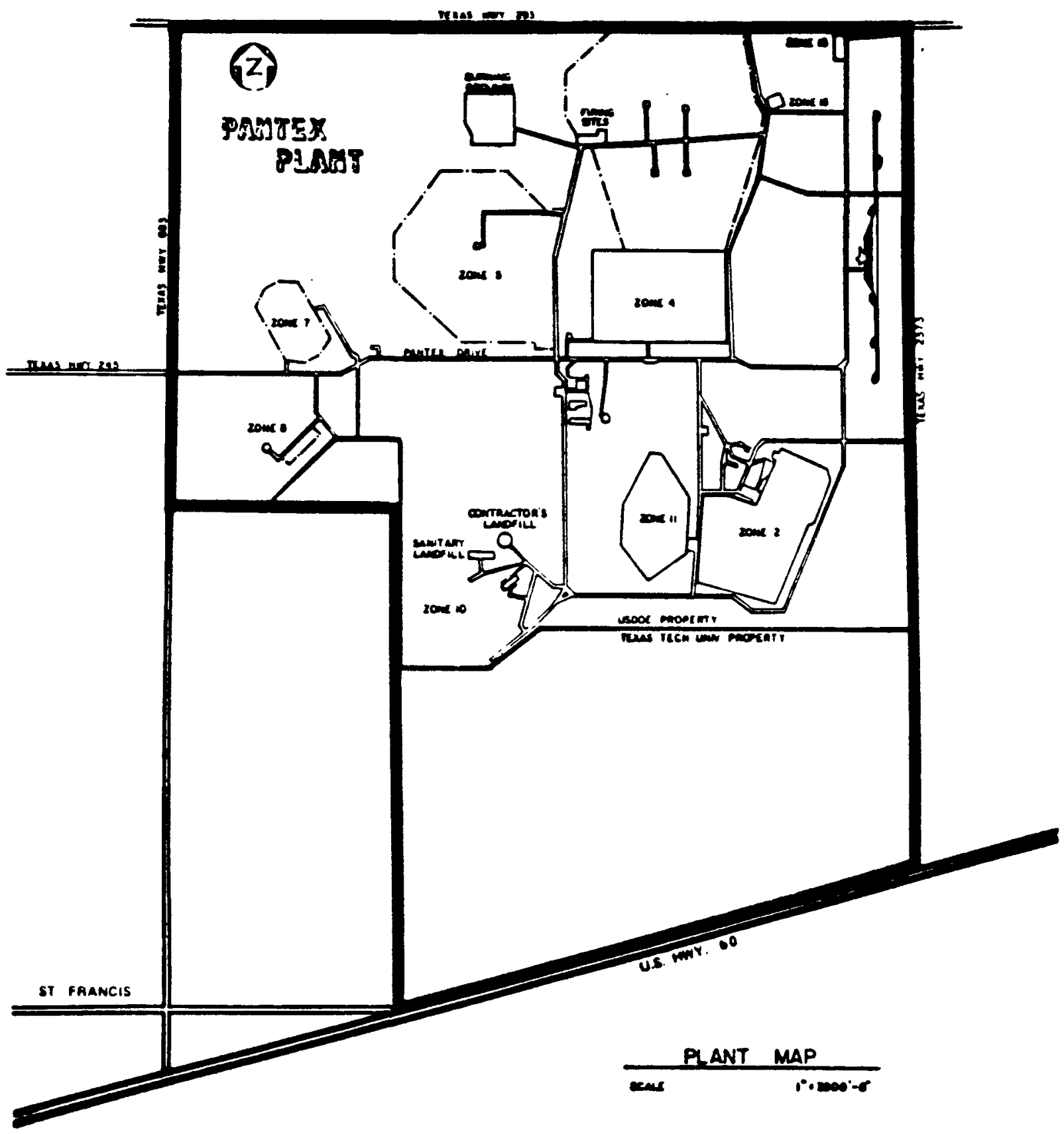

Figure 2. Pantex Plant Facility Site

The principal operation performed at Pantex Plant is the assembly of nuclear weapons. The overall plant mission involves the fabrication of chemical high explosive (HE) 
components for nuclear weapons, assembly and disassembly of nuclear weapons, modification and repair of nuclear weapons, and surveillance testing and disposal of chemical HE. Weapons assembly and stockpile surveillance activities involve handling significant quantities of uranium, plutonium, and tritium, as well as a variety of nonradioactive toxic chemicals.

Nuclear components are received at Pantex Plant from other DOE plants. These components require no further processing at Pantex Plant before being assembled into weapons, thus presenting very low probability of releasing radioactive materials during normal operating conditions. Radioactive material releases at Pantex Plant are limited to certain infrequent but routinely scheduled HE test fires, which disperse small quantities of depleted uranium (essentially U-238), and other operations within the plant which involve the release of small amounts of tritium (H-3). These airborne emissions of radioactive material (Table I) are well below prescribed standards. Likewise, nonradioactive emissions (Table II) from the on-site steam generating plant are below prescribed standards. A comparison of the Vicinity Dose from Pantex Plant (Table III) with the Vicinity Population Dose Due to Natural Occurring Radiation (Table IV) will verify how small Pantex releases are.

Pantex plant processed approximately 1.1 million liters $(300,000$ gallons) of sanitary wastewater per day during 1988. The treatment consists of a "facultative lagoon" biological treatment system. The treated effluent is used by Texas Tech University for irrigation purposes. This process is controlled by the Texas Water Commission (TWC), via their Wastewater Permit No. 02296.

All scrap paper and food discards from the cafeteria, office areas, and manufacturing areas are bagged in plastic and buried at the Sanitary Landfill, a 3-hectare (8-acre) site surrounded by a cyclone fence. No explosives, explosive-contaminated materials, or radioactive or hazardous waste are included in the landfill waste materials. All waste placed in the landfill is covered with dirt on a daily basis. No contamination of the aquifer water below the landfill area is anticipated because of the impervious nature of the topsoil and caliche layers and the low liquid content of the buried materials.

Waste and excess explosives are presently destroyed by burning in brick-lined pans in accordance with approved DOE and Department of Defense (DOD) methods of disposal of explosives(1,2). The explosives material, with a small amount of fuel oil added to assist in ignition, is placed on the pan. An explosive squib is connected from a wiring post to the waste material and the material is then squib-ignited remotely from the control bunker after personnel have cleared the area. HE-contaminated solvents (such as toluene, acetone, and methanol) are allowed to evaporate in an open tank, and the residue is burned. The burning of HE at Pantex Plant is approved by a written grant of authority issued by the State of Texas Air Control Board(3).

Low-level radioactive waste (LLW) solid material is collected, packaged, and shipped to Nevada Test Site (NTS). This waste consists of contaminated filters and paper wipes.

Chemical disposal is based on the nature of the chemical itself. Nonhazardous solid wastes are buried in the sanitary landfill. Resource Conservation and Recovery Act (RCRA) hazardous wastes that cannot be treated are shipped to an Environmental Protection Agency (EPA) approved disposal agencies, according to current state and EPA regulations. Hazardous solid waste activities are conducted in accordance with our Interim Hazardous Waste Permit No. 50131, as administered by TWC. 


\section{ENVIRONMENTAL SETTING}

Pantex Plant is located between the North Central Plains and the Llano Estacado (Staked Plains) which forms part of the Great Plains (Figure 3). The main operating areas of the plant are at an altitude of 1,078 meters $(3,538$ feet). The area is characterized by rolling grassy plains and numerous natural playas. These playas are fed only by rainwater and melted snow and are therefore frequently dry. The total precipitation in 1988 in water equivalence was $67.7 \mathrm{~cm}$ (24.7 inches), as reported by the Amarillo Office of the National Weather Service. The mean annual eveporation is approximately $241 \mathrm{~cm}(95$ inches)(4).

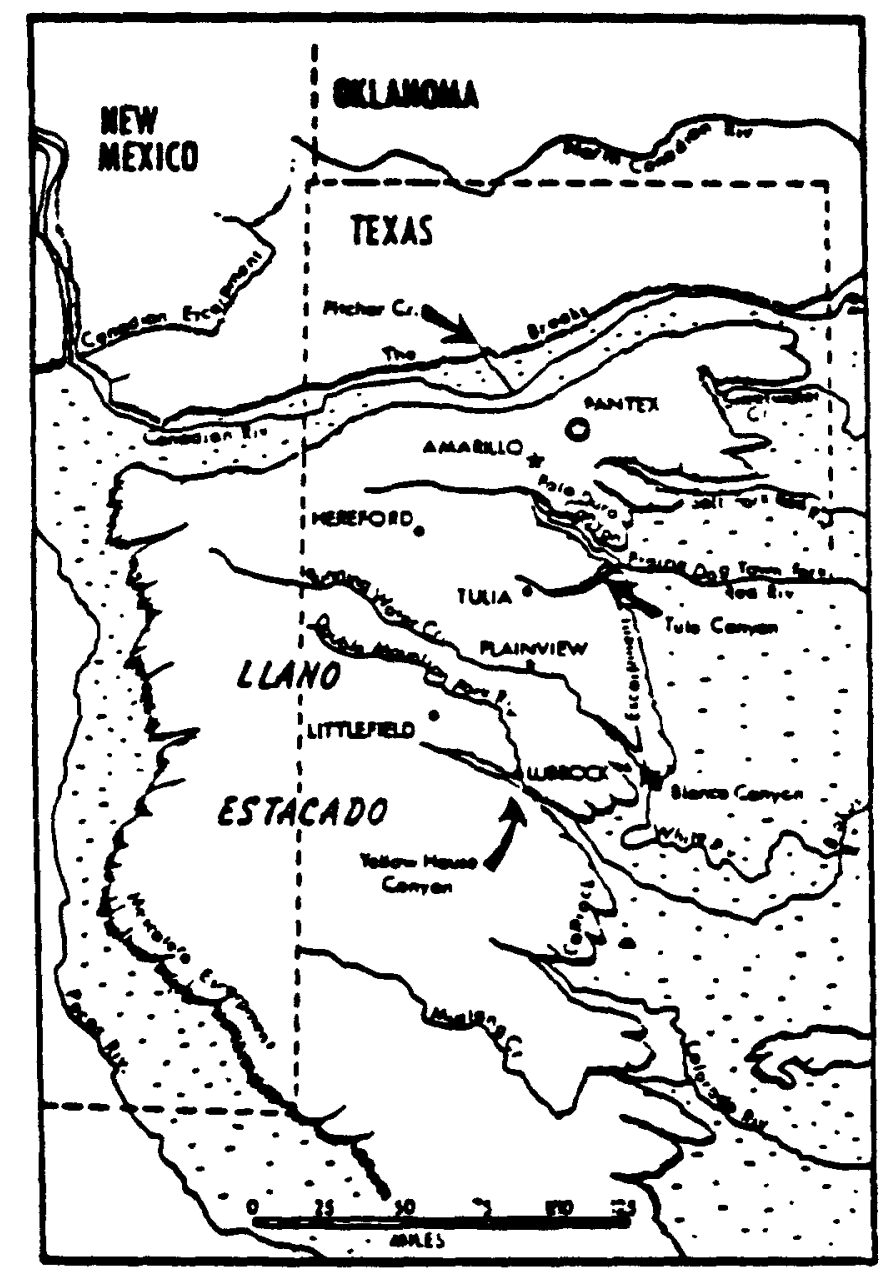

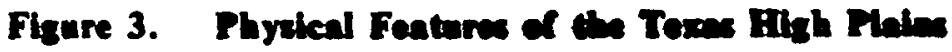

Local winds during 1988 were from the south and southwest directions approximately $40 \%$ of the time with an average ennual wind velocity of 5.9 meters per second (13.2 mph) (Figure 4). Pantex Plant is located in one of the more severe tornado areas of the United States. According to "Assessment of Tornados and Straight Wind Risks at the Pantex, Texas Siten(5), the probability of the plant site being hit by a moderate tornado (67 m/s rotational velocity) is approximately one in 50,000 in one year and the probability of the site being hit by a severe tornado (112 m/s rotational velocity) is approximately one in $2,000,000$ in one year. 


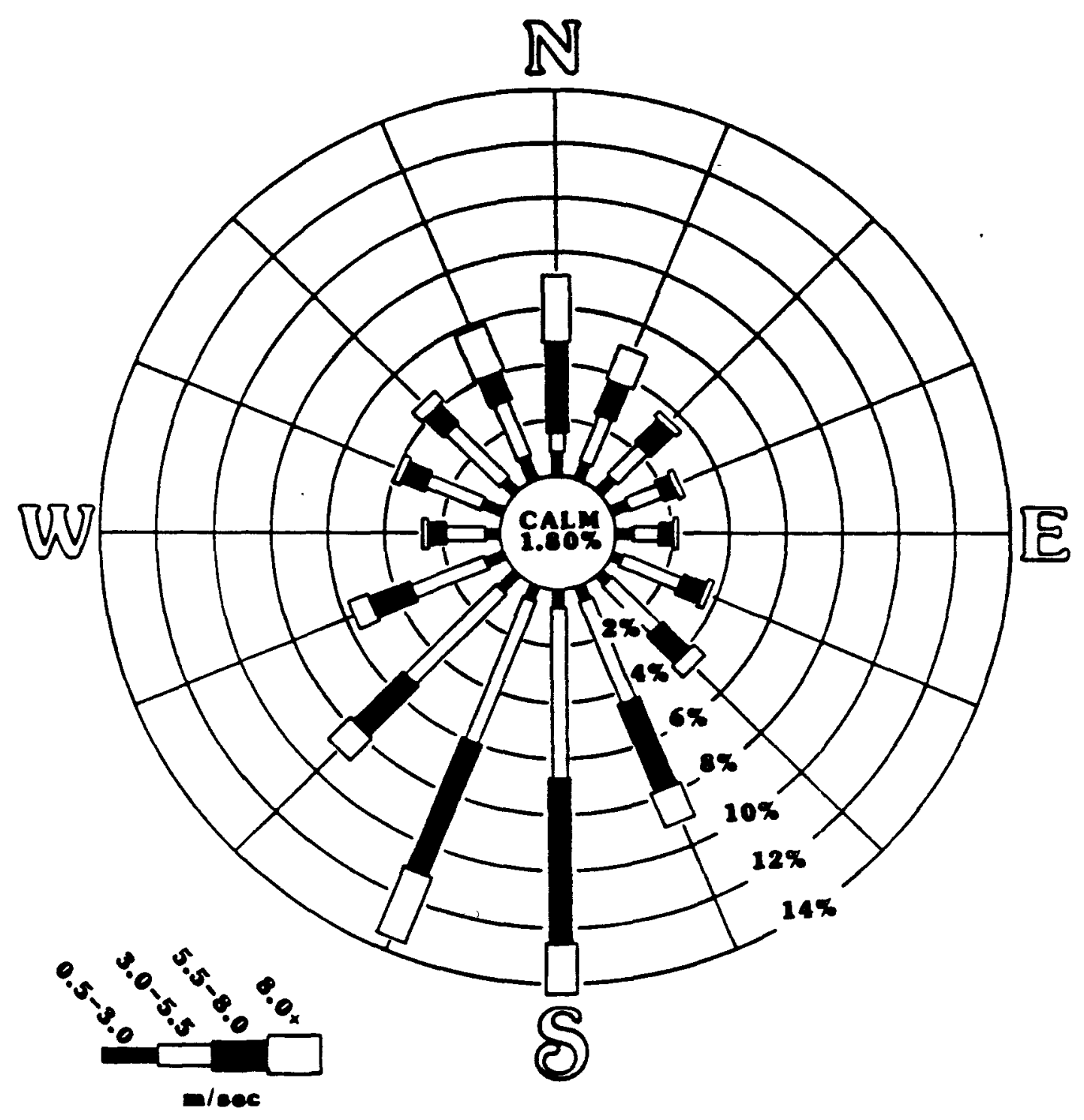

Figure 4. Anaud Anarillo Whad Rece for 1988

The Pantex location and surrounding area is relatively free of earthquakes. Since 1882 there have been four earthquakes in the area of the plant. These seismic events occurred on March 27, 1917; July 25, 1925; June 19, 1931; and July 20, 1966 . Their epicenters were sixteen to thirty-two kilometers (ten to twenty miles) east to northeast of the plant site, and all were estimated to have intensities of V or VI on the Modified Mercalli Intensity Scale(6). The Uniform Building Code places the Pantex area in Zone 1, which rates second lowest in earthquake occurrence on a scale from Zone 0 to Zone $4(7)$. 
Underlying the Texas Panhandle is the Ogallala aquifer which serves as the source of water for Pantex Plant and a large part of the water for Amarillo. Pantex Plant pumped 1.27 billion liters (337 million gallons) of water from the aquifer during 1988, while the City of Amarillo pumped 18.2 billion liters (4.9 billion gallons) from their Carson County field, immediately north of Pantex Plant. This withdrawal of water by the City of Amarillo causes the ground water in the main aquifer beneath Pantex Plant to move to the north rather than down gradient to the east. The saturated Ogallala aquifer is from 61 to 122 meters (200 to 400 feet) thick and at a depth below the surface of approximately 244 meters ( 800 feet). A surface layer of clay 18 to 24 meters (60 to 80 feet) deep forms a barrier to surface moisture and prevents deep penetration of surface contaminants $(8,9,10)$. This clay layer is usually underlaid with caliche and dry ogallala sands which overlay the saturated aquifer. Aquifer water which is removed is only partially replaced through naturd recharging processes(11).

The Panhandle area is not densely populated having approximately 255,000 persons residing within an 80 kilometer (50 mile) radius of the Pantex Plant(12). Amarillo, Texas, located southwest of the plant site, is the largest single populated center with about 150,000 residents. Pampa, Texas, located about 64 kilometers (40 miles) northeast of the plant, is second in population with about 21,000 residents. The remaining population is distributed throughout smaller communities, farms, and ranches. Although there are a variety of industries in the area, the local economy is influenced most significantly by agriculture and related industries. Privately-owned land in the immediate vicinity of the plant is used for farming and cattle ranching.

\section{ENVIRONMENTAL ACTIVITIES}

Pantex Plant had only one accidental release into the environment during 1988 . On May 131988 less than five gallons of sulfuric acid was spilled onto the ground from a defective pump seal. The spill was contained and neutralized immediately.

The following shipments of hazardous waste were made periodically throughout the year:

On July 28, 1988 approximately 22,000 pounds of waste barium nitrate "mock explosive," were shipped to Rollins Environmental Services, Deer Park, Texas.

Approximately 8,000 pounds of waste compound, naptha, cleaning liquid, and paint related material were shipped to Safety-Kleen Corporation, Amarillo Texas, for recycle.

Approximately 6,500 gallons of waste oil were shipped to Double Eagle Refining, Oklahoma City, Oklahoma.

Approximately 136,000 pounds of asbestos-covered piping were shipped to the Amarillo City Landfill, Amarillo, Texas.

Approximately 1,700 pounds of silver from film processing byproducts were shipped to Oklahoma Industrial Silver, Norman, Oklahoma. 


\section{ENVIRONMENTAL PROGRAM}

During 1988, Pantex Plant continued its environmental monitoring program which evaluated air, water, soil, vegetation, and specific biota samples. Analyses of area samples were specific for plutonium-239, uranium-234 and -238, and tritium, as these are the isotopes of concern in the assembly of nuclear weapons. Analyses were also performed for naturally occurring radium-226 and radium-228 as a result of requirements in the Safe Drinking Water Act.

Isotopes common to nuclear reactor sites such as strontium-90 and cesium-137 were not monitored at Pantex because there are no nuclear reactors or high level radioactive waste from nuclear reactors at Pantex Plant.

There were ten off site air sampling locations (Figure 5 ) and seven plant site locations (Figure 6) used during 1988 in our air sampling program. Nine off site and all seven plant site stations were equipped with continuously operating low and high volume samplers. The one remaining off site location, which was our background sampler, was equipped with a high volume sampler. This sampler was located at the Bushland Agricultural Research Station, which is located west of Amarillo, as noted on Figure 1.
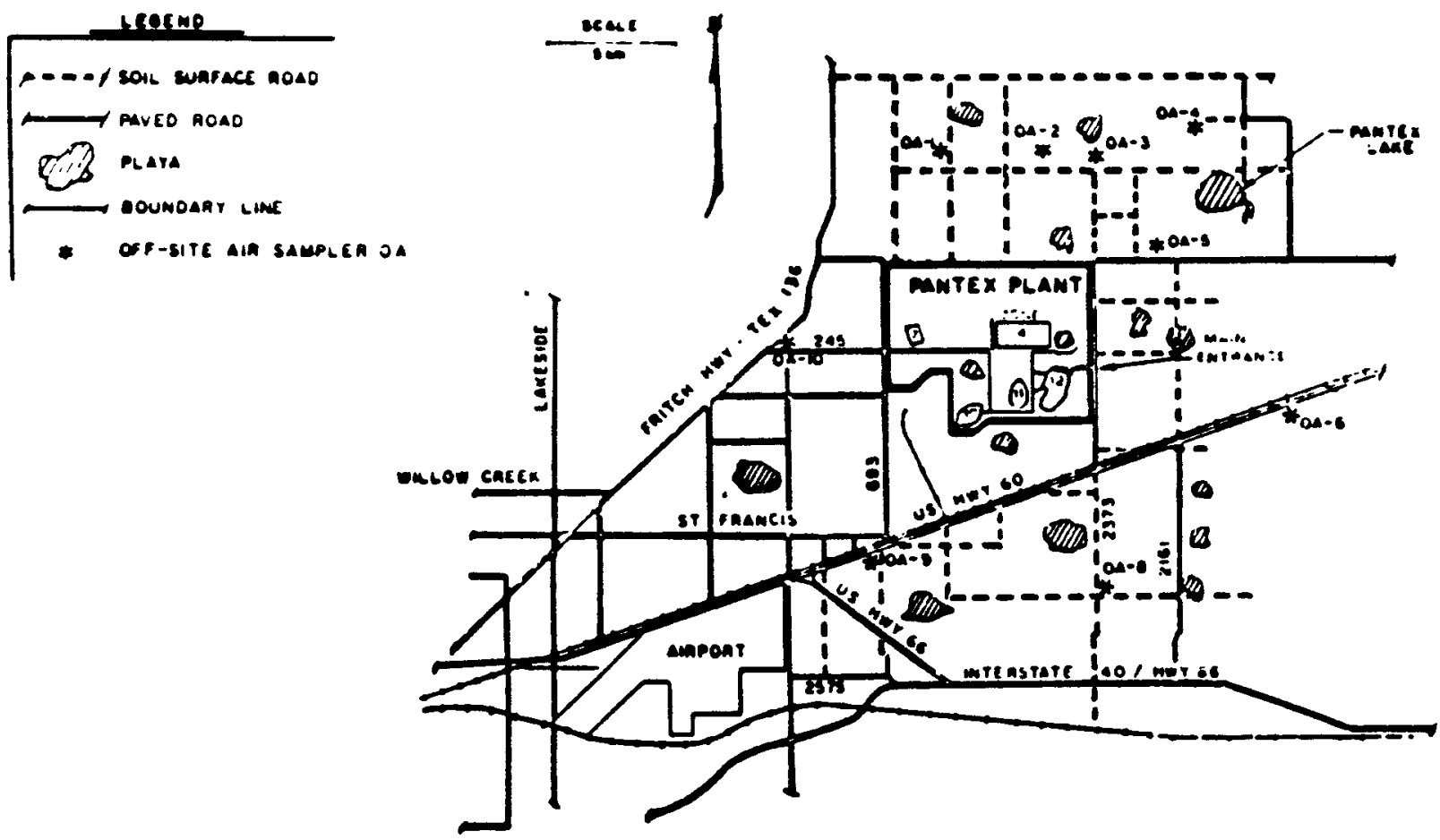

Tgure 5. Off Eite AIr Sampling Leentien 


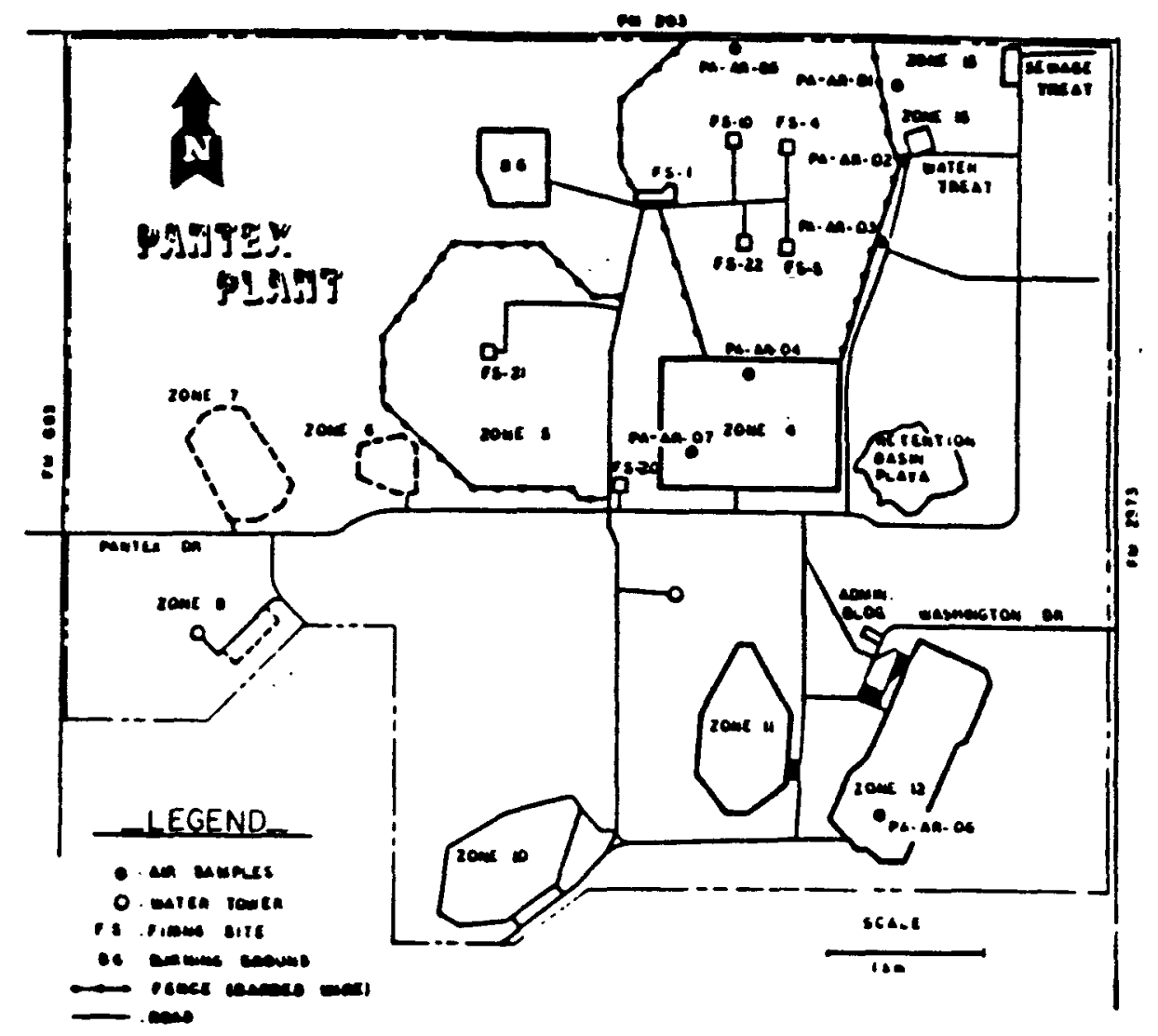

Figure 6. Plant Site Air Sampling Locations

Air samples collected in 1988 were analyzed for uranium-234 and -238 , plutonium-239, and tritium. The data are presented in Tables $V$ through VIII. In no case were any of the results in excess of the established radioactivity concentration guides (RCG) or derived concentration guides (DCG).

Water sampling was performed on ground waters from Pantex wells, on surface waters at plant site retention playas and treated sanitary effluents prior to use in crop irrigation. Samples were also collected from plant drainage ditches. The drainage ditches are referred to as "Industrial Waters" in the table section of this report. The various ditches flow into two plant site playas, OW-WR-08 and OW-WR-27.

The data on radioactivity in water, as presented in Tables IX through XVII, indicate the absence of radioactivity discernible above natural levels. Also, the data indicate conformance of our drinking water with maximum contaminant levels for radioactivity in drinking water, as defined in 40 CFR 141.

Soil samples were collected and subjected to analysis for beryllium, uranium-234 and238, and plutonium-239 content at locations indicated on Figures 1, 7, 8, 9, and 10. Results are presented in Tables XIX through XXII. 


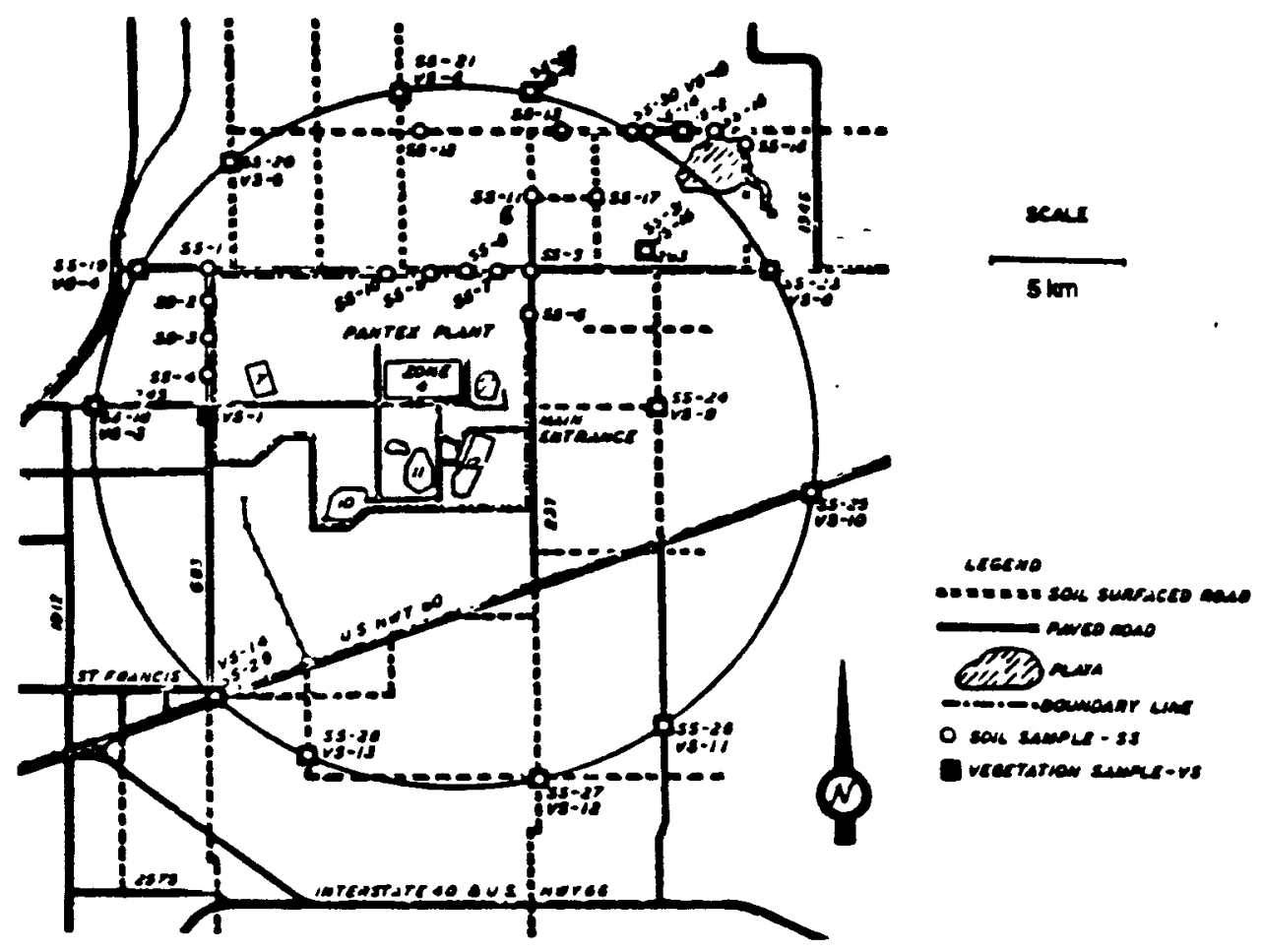

Figure 7. Off Site Eavironmental Soll and Vegetation Sampling Locations for Pantex Plant [8-kilometer (5-mile) Radius]

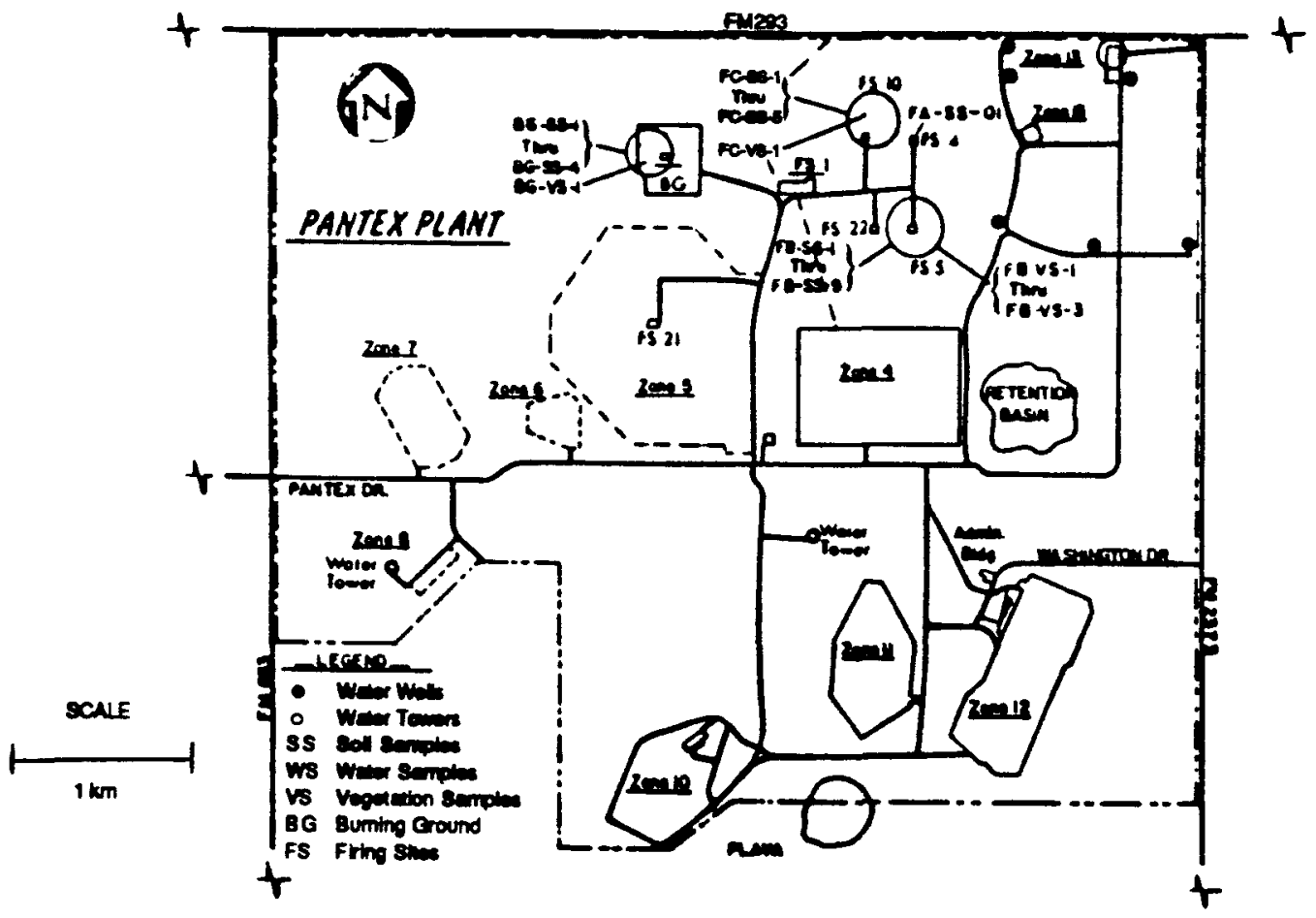

Figure 8. Plant Site Soll and Vegetation Sanpling Locations 


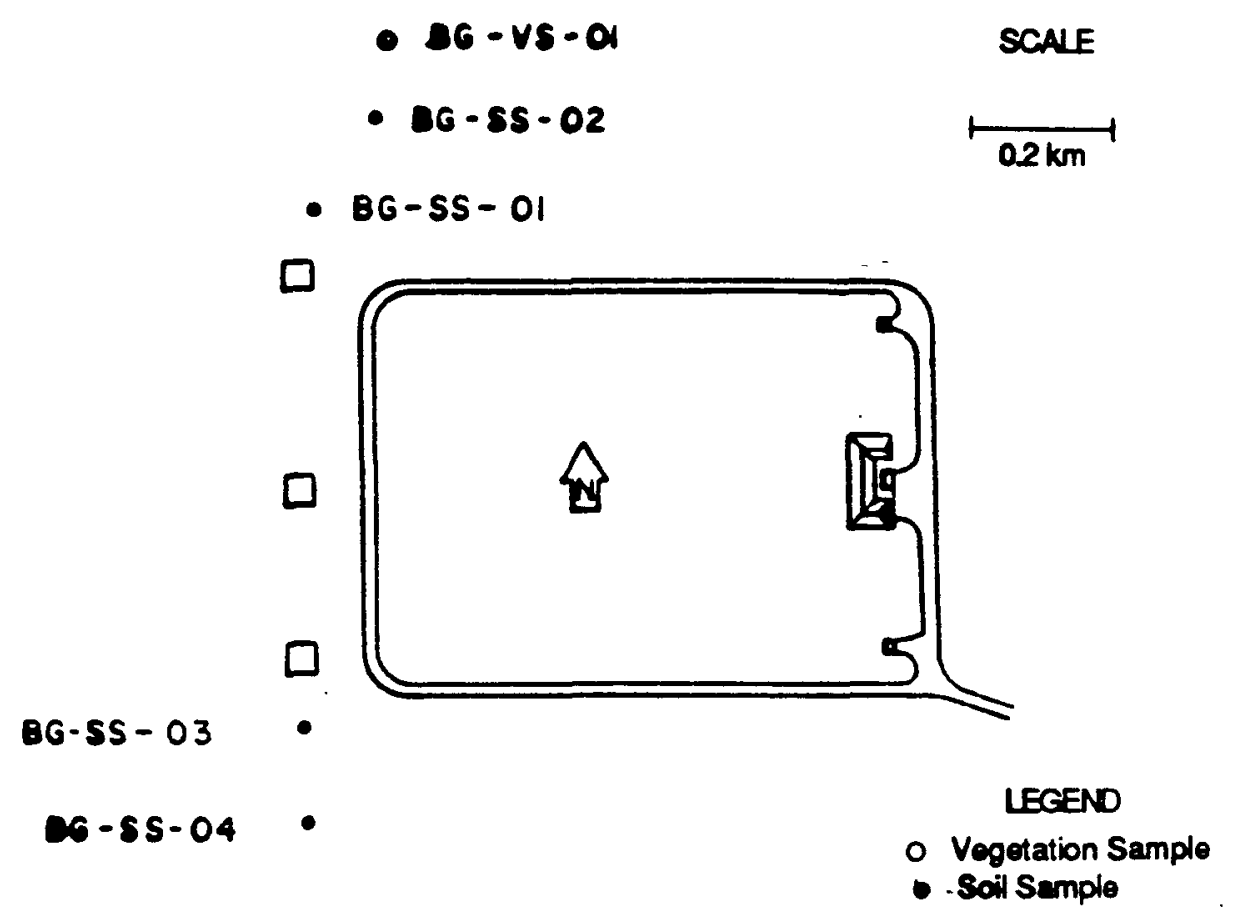

Figure 9. Buraing Ground Soil and Vegetation Samplieg Locations

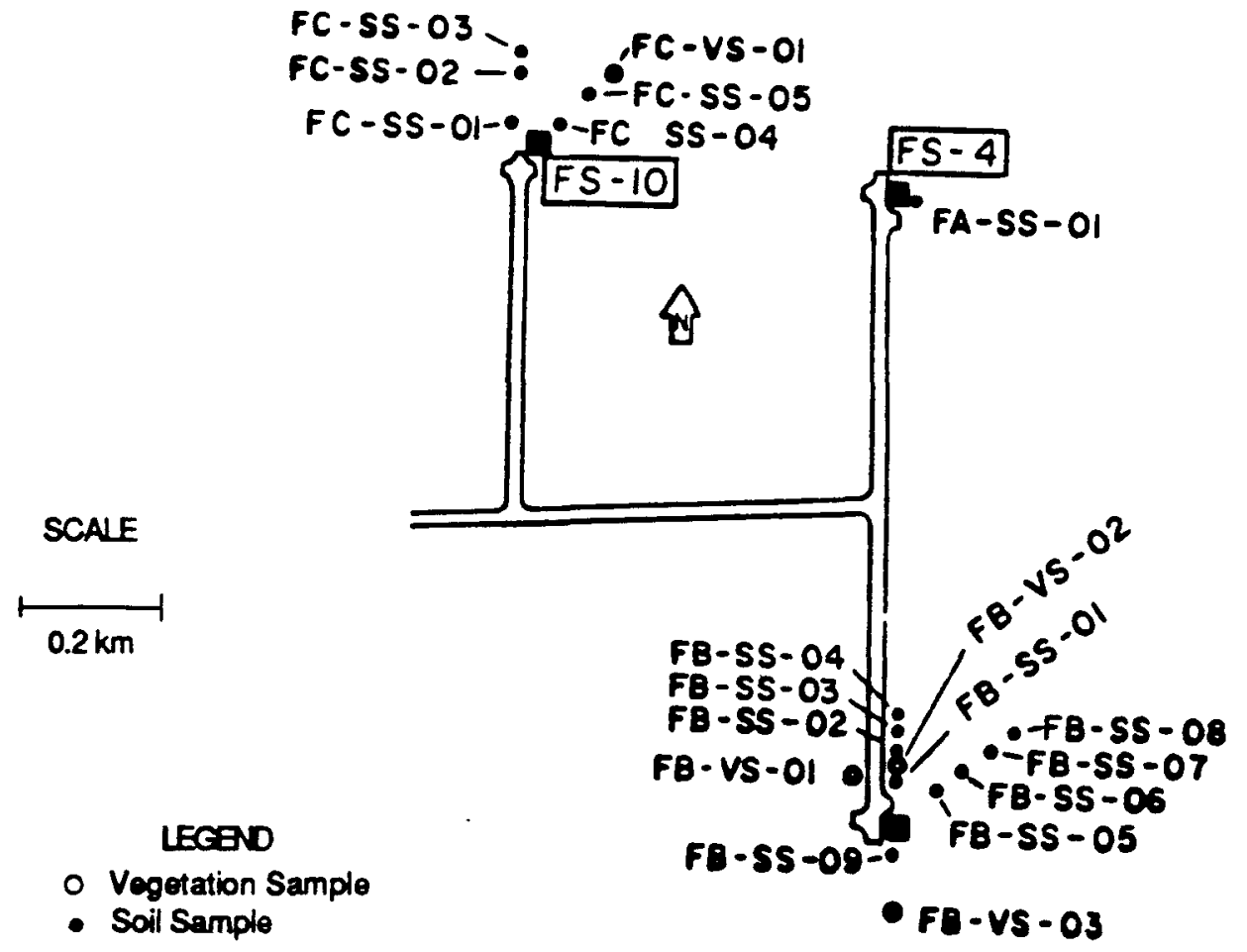

Figure 10. Firing Site Soll and Vegetation Sampling Locations 
Beryllium in soil data is presented in Table XIX. There are no concentration guides for radionuclides in soil. The concentrations presented in Tables $\mathbf{X X}$ and $\mathbf{X X I I}$ are consistent with data published in earlier reports, indicating no upward trend.

Analysis of vegetation to determine uranium-234 and -238 , and tritium concentrations was performed to determine whether unusual uptake of uranium or tritium from the soil or air had occurred. As with soil, there are no concentration guides for uranium or tritium in vegetation, although concentration guides for water are sometimes used for purposes of comparison.

Since 1974, the jackrabbit (Lepus californicus gray) has been used for analysis in the environmental monitoring program. The jackrabbit was chosen as the subject because of its normal abundance, its prolific nature, and its habit of maintaining a limited range as long as food is available.

Four types of analyses were performed on jackrabbits as presented in Tables XXVII through XXXIII.

AIR

Radioactive

Off site environmental air sampling is accomplished through the use of 18 continuously operating air samplers located at nine sites around an approximate 8-kilometer (5-mile) radius of Pantex Plant. Each sample site on the 8-kilometer radius around Pantex Plant is outfitted with a high volume and a low volume sampler. $A$ high volume sampler was stationed at the United States Department of Agriculture (USDA) Bushland Research Station, which is located about 20 kilometers $(12$ miles) west of Downtown Amarillo (Figure 1). This sampler is our background sampler. Plant site sampling is accomplished through 14 samplers located at the seven sites shown on Figure 6.

The high volume samplers, which are equipped with automatic flow-rate controllers, operate at a sampling rate of approximately 1.1 cubic meters/minute $(40 \mathrm{cfm}$ ). Particles are collected on $20 \times 25 \mathrm{~cm}(8 \times 10$-inch) filters. The filters are changed on a weekly basis, weather permitting. A weekly air sample represents about 11,400 cubic meters.

Each weekly air filter is composited on a bimonthly basis with other filters from the same air sampling station. These samples are then sent to an outside laboratory for uranium-234 and -238 and plutonium-239 analysis.

Each low volume sampler employs a silica gel column in the sampling line for collection of environmental tritium oxide. The silica gel, acting as a desiccant, removes water vapor from the air stream. Any tritium oxide present will be captured with the water vapor and recovered for analysis. Silica gel columns are exchanged on the same frequency as the air filters. Nominal air flow is about 42.5 liters per minute (1.5 $\mathrm{cfm}$ ). Silica gel samples are analyzed by an outside laboratory to determine tritium content.

Summaries of the results for uranium-234 and -238 , plutonium-239, and tritium in air are presented in Tables $V$ through VIII. Deviations above background are attributed 
Summaries of the results for uranium-234 and -238 , plutonium-239, and tritium in air are presented in Tables $V$ through VIII. Deviations above background are attributed to natural meteorological distribution of worldwide fallout.

\section{Nonradioactive}

Pantex Plant does not have any industrial processes which serve as significant sources of particulate matter, carbon monoxide, photochemical oxidants, hydrocarbons, oxides of nitrogen, or oxides of sulfur. Steam is generated by natural gas-fired boilers.

The estimated releases in Table II are based on emission factors provided by the Texas Air Control Board (TACB), and 40 CFR 60.40 (Appendix, Page A-9). Other sources of pollutants are generally absent since most of the work performed is mechanical rather than chemical. Estimated emissions from Pantex Plant steam generation operations during 1988 are presented in Table II.

Some emissions occur from test fires of chemical high explosives, burning of waste high explosives, and flashing of high explosives-contaminated scrap metal. Approximately $68,000 \mathrm{~kg}(149,800 \mathrm{lbs})$ of waste chemical high explosives were burned, and approximately 19,065 liters $(5,037$ gallons) of mixtures of volatile organic solvents (toluene, acetone, methanol, etc.) and water were disposed of by evaporation during 1988. Calculations done during preparation of the environmental impact statement indicate that these emissions were within guidelines and are still within guidelines.

\section{WATER}

\section{Radioactive}

Local hydrologic characteristics of the Pantex vicinity are such that there are no streams or rivers which flow through or near the plant site. Plant effluents flow via drainage ditches ("Industrial Waters"), into two plant site playas (designated OW-WR-08 on Figure 11) prior to use for irrigation, and a second plant site retention playa (OW-WR-27) which is used for collection of plant effluents from Zone 11.

These plant site retention playas and groundwater from plant wells are used for collecting environmental water samples. Plant wells include deep water wells into the Ogallala Aquifer and two special shallow test wells designated OW-WR-19 and OW-WR-20. OW-WR-19 is located so as to indicate whether contamination from the retention playa is leaching into the groundwater.

Waster samples are collected monthly and sent to an outside laboratory for analysis of uranium-234 and -238, plutonium-239, radium-226, radium-228, and tritium content. These samples were also analyzed for dissolved and suspended alpha, and dissolved and suspended beta. Summaries of these data for 1988 are presented in Tables DX through XVIII. The QA Program for this laboratory is presented in Appendix A. 


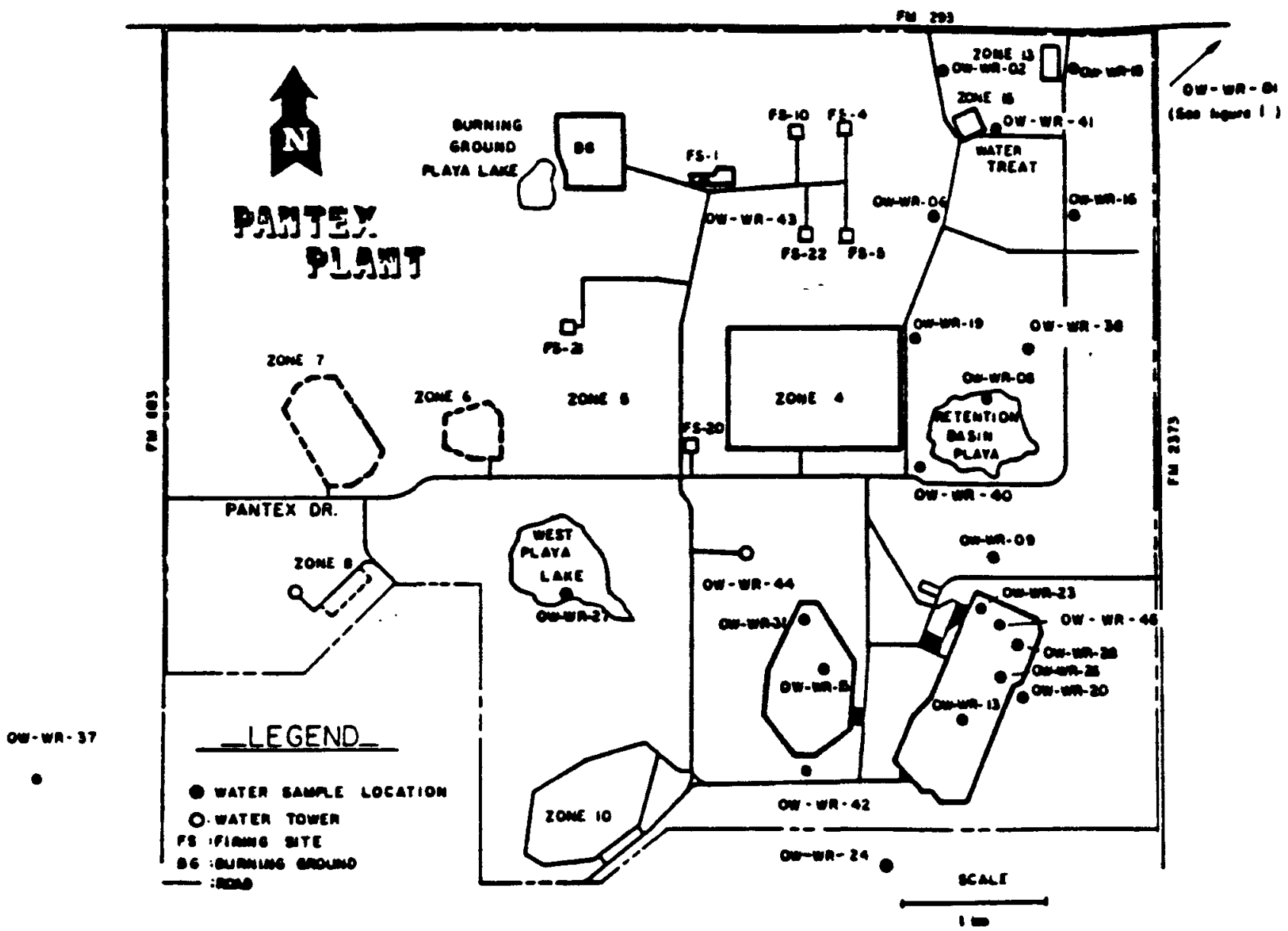

Figure 11. Plant Site Water Sampling Locations

\section{Nonradioactive}

These same environmental water samples are also analyzed for nonradioactive species.

A summary of analyses for nonradioactive species for 1988 is presented in Table XXXIV.

All test data were within applicable regulations. Biochemical oxygen demand (BOD) and $\mathrm{pH}$ on sewage treatment plant discharge and chemical oxygen demand (COD) and pH on industrial discharges are controlled by the Texas Water Commission Permit No. 02296. See Table A-VII for listing of minimum criteria. 
SOIL

\section{Radioactive}

Thirty-two off site environmental soil samples were collected quarterly, weather permitting, at the locations indicated in Figure 7 according to standard soil sampling protocol. Each sample consisted of plug from a cookie cutter which is 8.9 centimeters in diameter and 5.1 centimeters in depth. Each sample may, also have contained a small amount of roots/vegetation.

Seventeen plant site soil samples were collected monthly at locations shown on Figures 8, 9, and 10. A control sample was also collected each month at the USDA Bushland Site, as shown in Figure 1.

After collection, the samples were sent to an outside laboratory for analysis of each isotope of interest.

Summaries of soil sampling results are presented in Tables XX through XXII for uranium-234 and -238 , and plutonium-239.

Sample site FC-SS-05 for uranium-238 in soil shows high results. Data history has shown this and the immediate area to have occasionally high readings due to depleted uranium from past test firings.

\section{Nonradioactive}

Soil samples from two plant site HE test fire sites (Figure 10) were analyzed for beryllium. Data is presented in Table XIX.

\section{VEGETATION}

\section{Radioactive}

Environmental vegetation samples were collected from seventeen off site locations (Figure 7) and five plant site locations (Figures 8, 9, and 10) for analysis of uranium -234 and -238 , and tritium. Off site samples and plant site samples were collected monthly. These samples consist primarily of native and domestic grasses, although it is not uncommon for a small percentage of other vascular plants to be present in some samples.

Following collection and labeling, the samples were sent to an outside laboratory for the actual analysis. The analytical procedures used were essentially the same as for analysis of radionuclides in soil. Summaries of the results are presented in Tables XXIV through XXVI.

Sample site OV-VS-04 for uranium-234 in vegetation shows high results. All three samples were double checked by an independent lab. Two samples were within guidelines while the third was not. Data history has shown no high readings for this site. Taking the data history and double check into consideration, the third sample is assumed to be an anomaly. 


\section{BIOTA}

\section{Radioactive}

During 1988, ten jackrabbits (Lepus californicus sray) were collected from within the controlled plant area and analyzed for the presence of uranium-234 and -238, plutonium-239, and tritium. The locations where the epecimens were collected were chosen carefully to help determine whether other-than natural levels of uranium-234 and -238 , plutonium-239, or tritium were present in the biological cycle.

Following collection, the specimens were frozen and sent to an outside laboratory for dissection and analysis. Analyses of lung, liver, kidney, bone, and muscle tissue were performed to determine the quantity of uranium-234 and -238, plutonium-239, and tritium present in each organ. These data are presented in Tables XXVII through XXXIII.

\section{CALCULATION OF POTENTIAL RADIATION DOSE TO THE PUBLIC FROM PANTEX ACTIVITIES FOR 1988}

The EPA has published regulations which limit the amount of airborne radionuclides released from DOE facilities. The EPA has specified that the AIRDOSE-EPA computer program shall be used to demonstrate compliance unless an alternate model has been approved by the EPA, (40 CFR 61).

Radiation exposure to the public from Pantex activities in minimal. Maximum individual and population dose from routine activities were estimated using the AIRDOSE-EPA computer program, (results in Table III). Population estimates were based on figures presented in the "Final Environmental Impact Statement, Pantex Plant Site, 1983" for projected 1990 population, (Figure 12). Meteorological data from 1988 was obtained from the National Oceanic and Atmospheric Administration NOAA, (Figure 4). Total radionuclides released for 1988 amounted to 0.121 curies of tritium to the air, with no releases to liquid effluents. The population estimates, meteorological data and radionuclide released were utilized as input parameters for AIRDOSE-EPA.

For Pantex operations, three groups of receptors were chosen in performing dose calculations: (a) hypothetical site boundary receptors, (b) nearest inhabitants to the plant reservation, and (c) cities, towns, and communities within an 80-kilometer (50-mile) radius of Pantex Plant. Dose estimates are expressed in rem or person-rem where the term person-rem refers to the product of effective dose equivalents times population for the various receptor groups. 


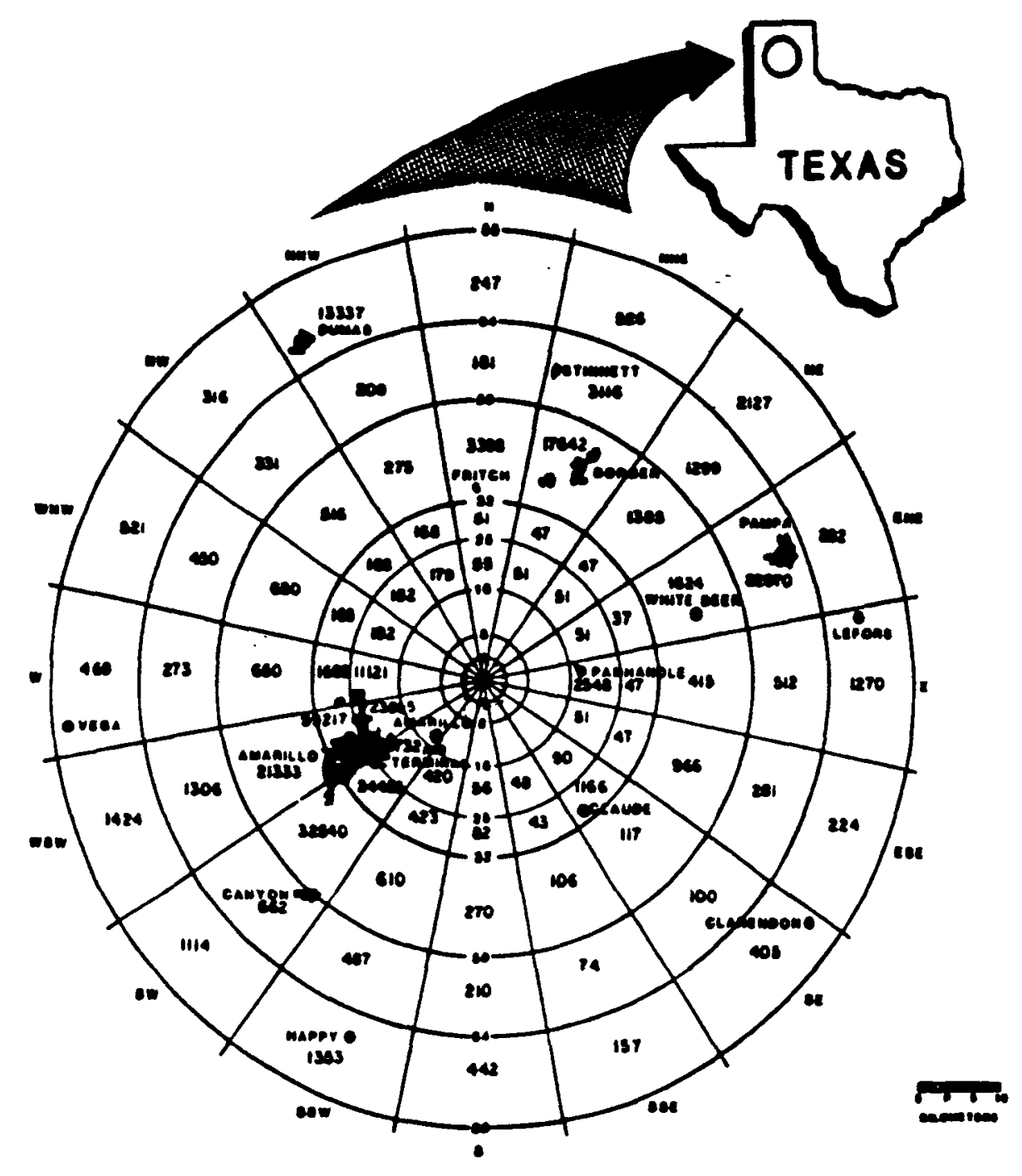

Figure 12. Population Within an 80-klloweter (50--ille) Radius of Pantex Plant

\section{SUMMARY}

An environmental monitoring program was conducted at Pantex Plant in 1988 for the purpose of detecting the possible release of radioactive material to the air, water (surface or ground), or soil, and for the purpose of detecting the release to waters of chemical contamination from plant operation. Data from this program is presented in Tables I through XXXIV. All releases were within applicable guidelines and regulations. Data from sites at the Bushland Agricultural Experimental Station is presented to show a background level for each analysis.

Total radionuclide releases for this year amounted to 0.121 curies of tritium to the air, and no releases to liquid effluents. This resulted in an estimated exposure to the maximally-exposed individual of $4.9 \times 10^{-9} \mathrm{rem}$, compared to a natural background level of $0.1 \mathrm{rem}$. This release resulted in a population exposure within 80 kilometers (50 miles) of $1.0 \times 10^{-5}$ person-rem, as compared to a natural background level of 3 $\times 10^{4}$ person-rem. 
Table 1. Estimated Atmospheric Releases of Radioactive Material from Pantex Plant for 1988

Nuclide Curies

\begin{tabular}{lc}
\multicolumn{1}{c}{ Nuclide } & Curies \\
\cline { 2 - 2 } Depleted Uranium (U-238) & 0 \\
Plutonium (Pu-239) & 0 \\
Tritium $(H-3)$ & $1.21 \times 10^{-1}$ \\
\hline
\end{tabular}

Table II. Estimated Steam Generating Plant Enissions for 1988

\begin{tabular}{lr} 
Contaminant & $\begin{array}{r}\text { Release } \\
(1 \text { bs) }\end{array}$ \\
\cline { 1 - 2 }$\quad \begin{array}{r}8,300 \\
\text { Carbon Monoxide }\end{array} \quad 47,500$
\end{tabular}

a.4 $4.10^{11}$ Btu's of gas fuel were used in 1988

Table III. Vicinity Dose Calculations from Pantex Plant Contributions for 1988

Reference Point

Nearest Individual

Site Boundary

Bo-Kilometer
Tritium ( $H-3$ )

(Total Body)

$4.9 \times 10^{-9}$ rem

$1.5 \times 10^{-8} \mathrm{rem}$

$1.0 \times 10^{-5}$ person-rem

Table IV. Pantex Vicinity Population Dose Due to Maturally Occurring Penetrating Radiation*

Reference Point

Dose

Total Body

$\begin{array}{ll}80-k i l a m e t e r & 3 \times 10^{4} \text { person-rem } \\ \text { Typical Individual } & 0.1 \mathrm{rem}\end{array}$

Based on an exposure rate of $13.3 \times 10^{-6} \mathrm{R} / \mathrm{hr}$ due to naturally occurring sources of penetrating radiation as measured by thermoluminescent field dosimetry. 
Table V. Summary of Tritium in Air for 1988

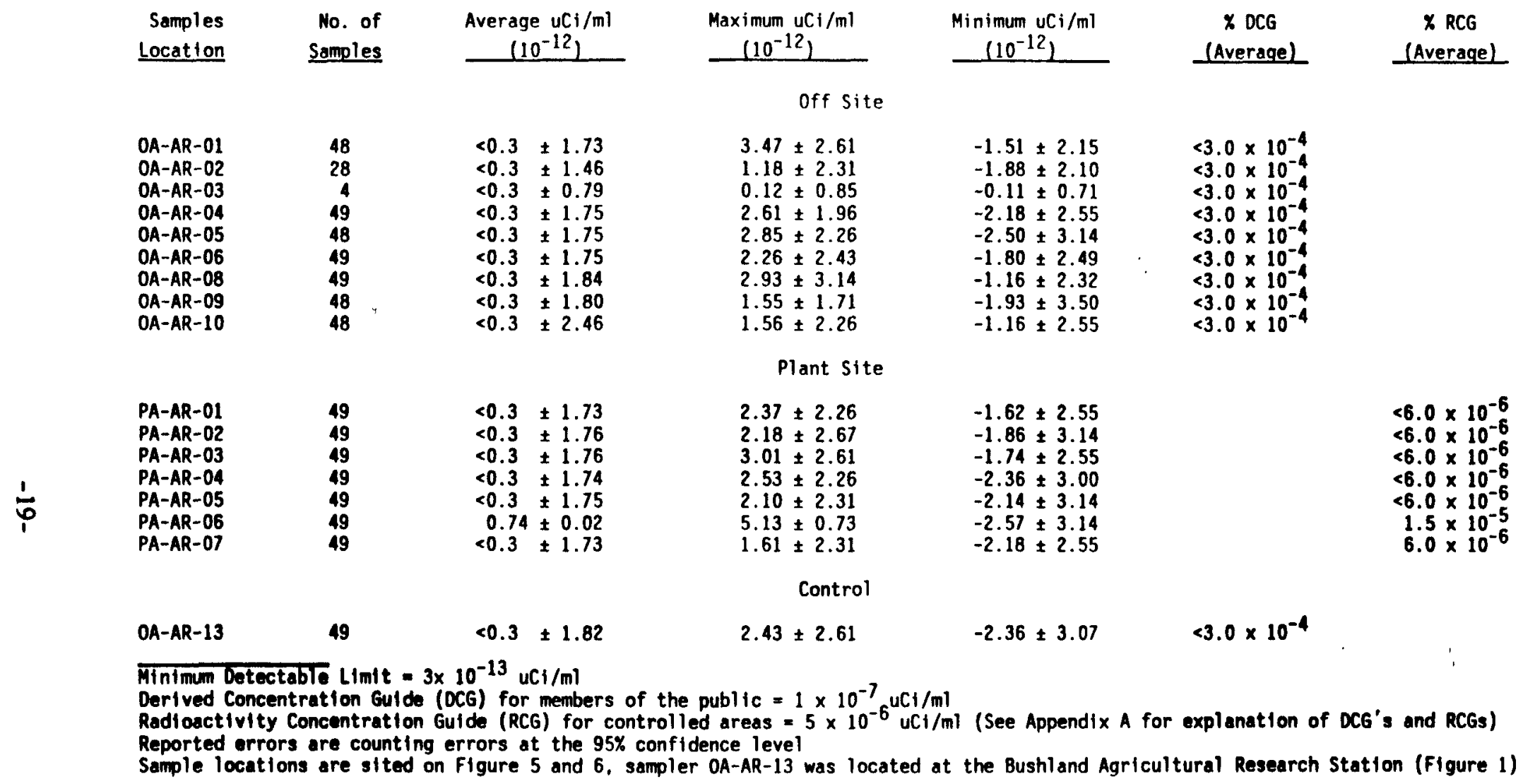


Table VI. Summary of Plutonium-239 in Air for 1988

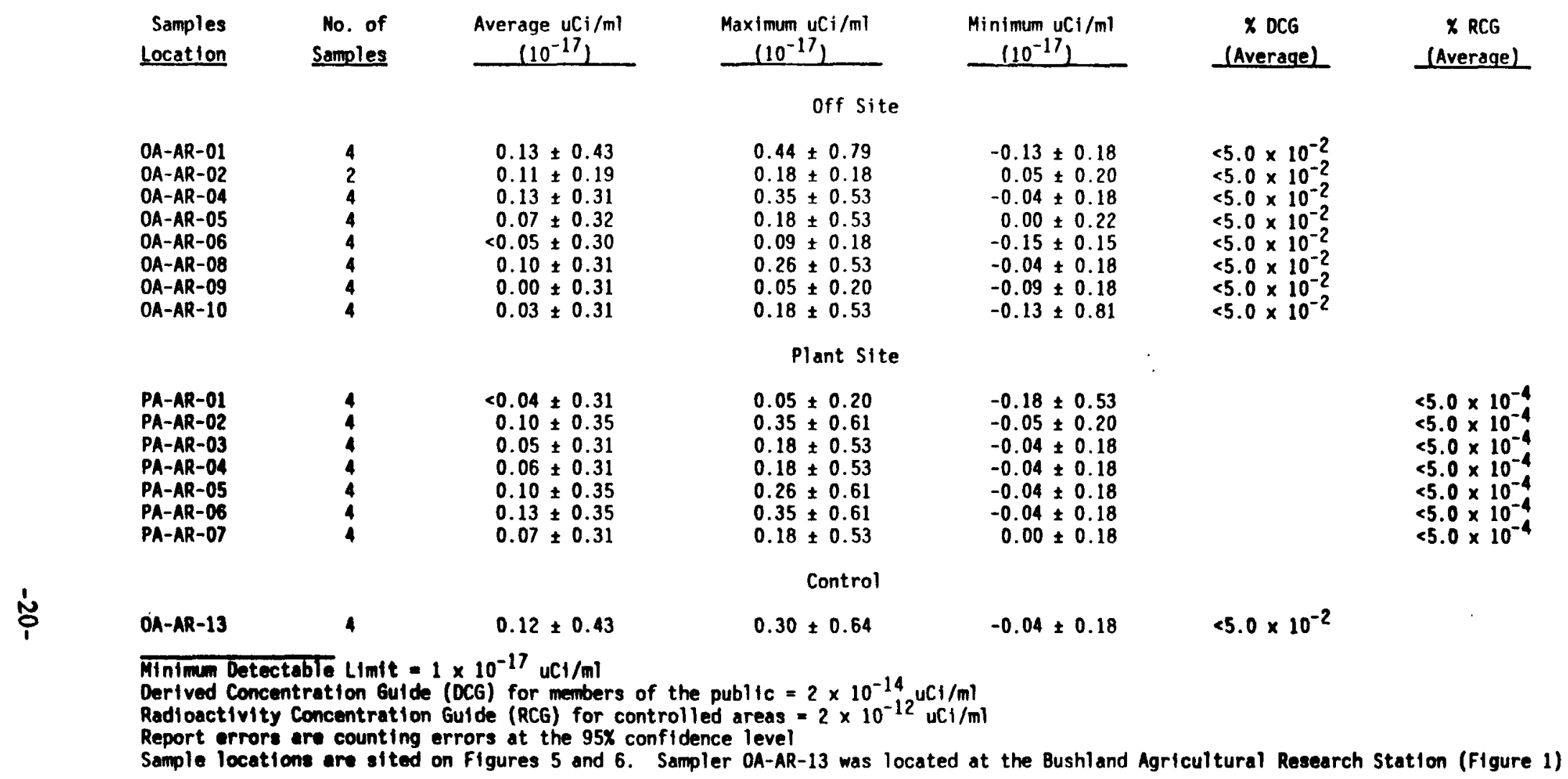


Table VII. Summary of Uranium-234 in Air

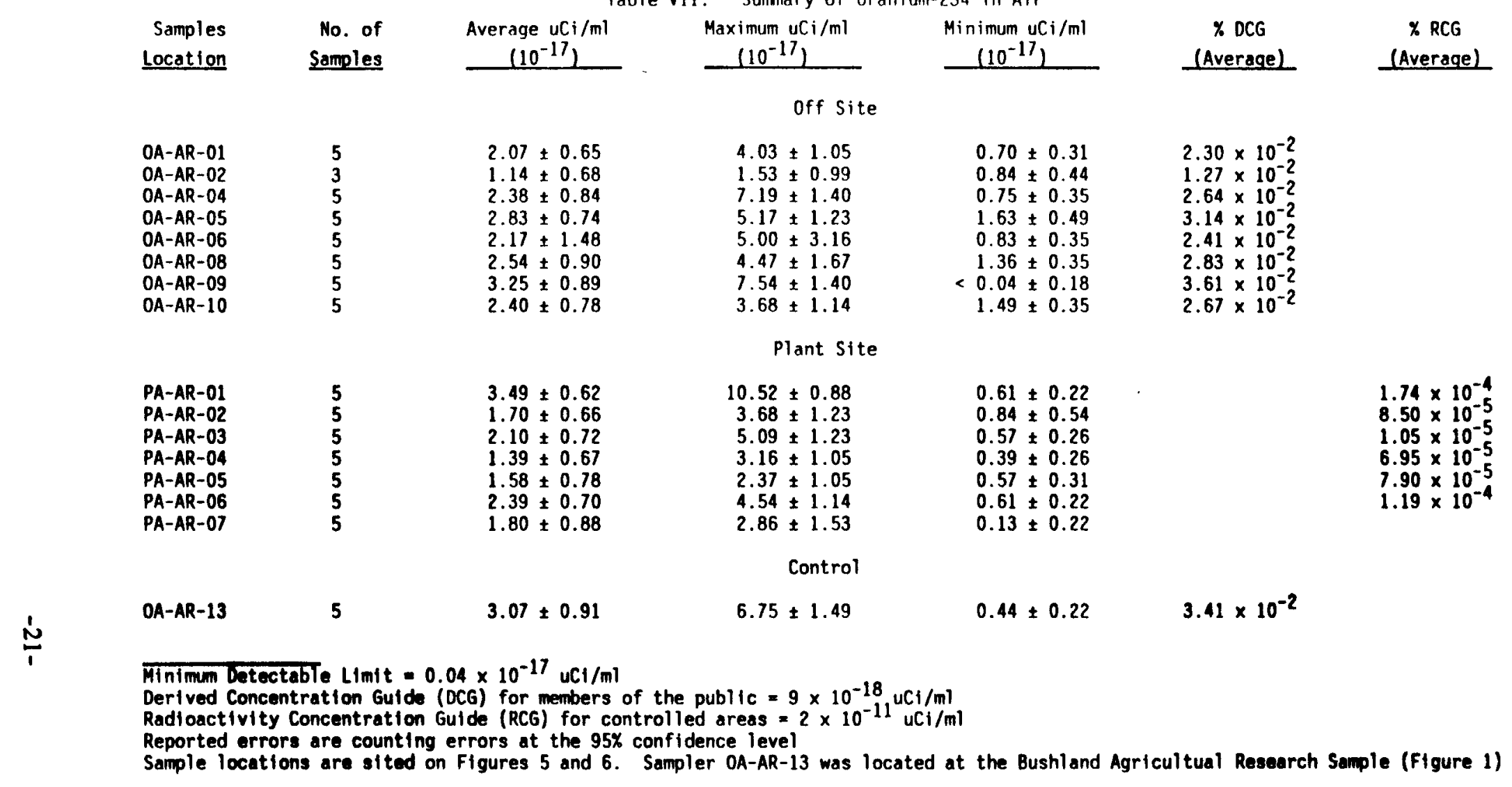


Table VIII. Summary of Uranium-238 in Air

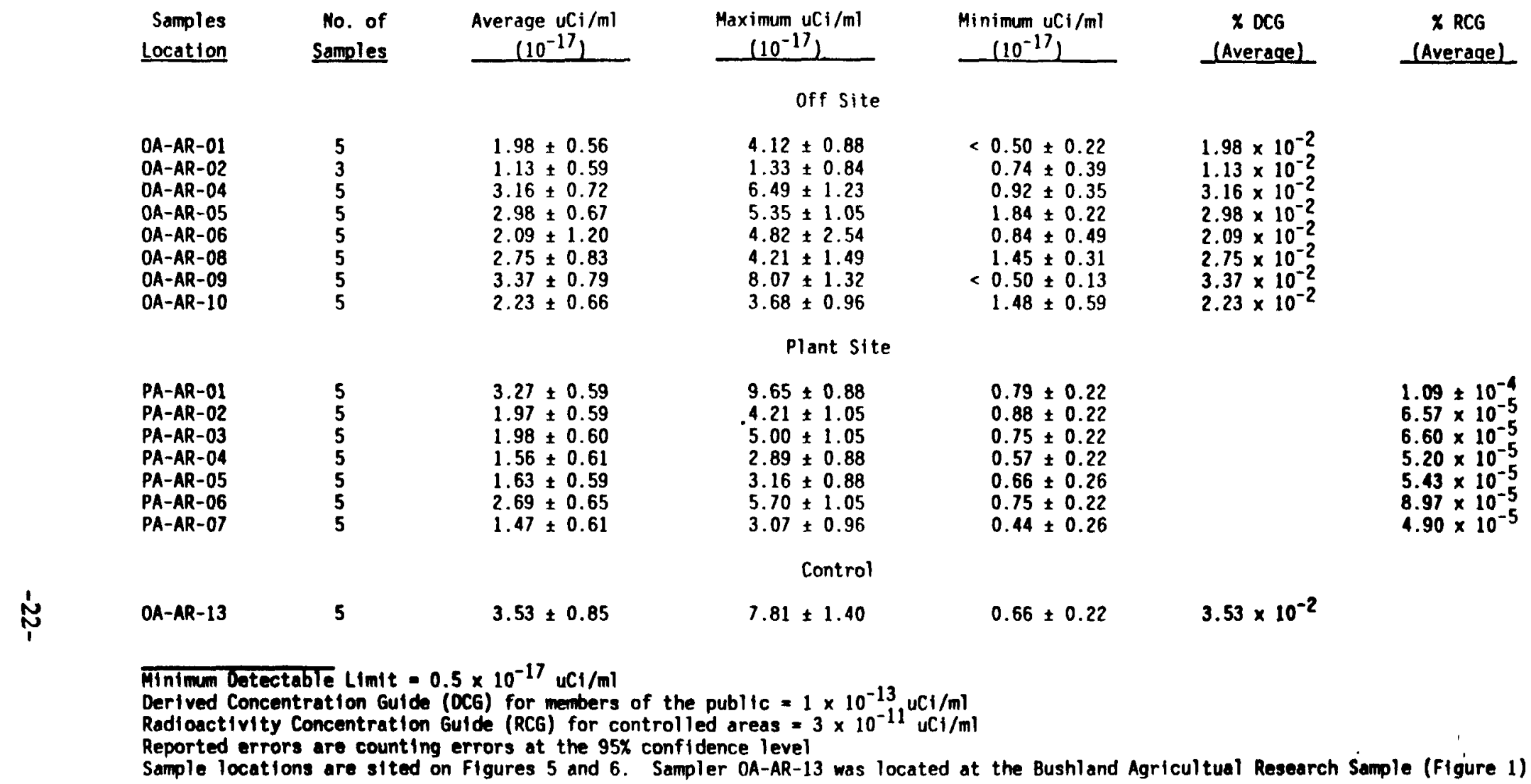


Table IX. Summary of Tritium in Water for 1988

\begin{tabular}{|c|c|c|c|c|c|c|c|}
\hline $\begin{array}{r}\text { Samples } \\
\text { Location } \\
\end{array}$ & $\begin{array}{l}\text { Ho. of } \\
\text { Samples }\end{array}$ & $\begin{array}{c}\text { Average } u C i / m) \\
\left(10^{-6}\right)\end{array}$ & $\begin{array}{l}\text { Maximum uCi/ml } \\
\left(10^{-6}\right)\end{array}$ & \multicolumn{2}{|c|}{$\begin{array}{l}\text { Minimum uCi/ml } \\
\left(10^{-6}\right)\end{array}$} & \multirow[t]{2}{*}{$\begin{array}{c}\times \text { DCG } \\
\text { (Average) } \\
\end{array}$} & \multirow[t]{2}{*}{$\begin{array}{c}\text { × RCG } \\
\text { (Average) } \\
\end{array}$} \\
\hline \multicolumn{6}{|c|}{ Playas } & & \\
\hline $\begin{array}{l}O W-W R-01 \\
O W-W R-08 \\
O W-W R-24 \\
O W-W R-27 \\
O W-W R-36\end{array}$ & $\begin{array}{r}9 \\
11 \\
11 \\
6 \\
6\end{array}$ & $\begin{array}{r}0.00 \pm 0.15 \\
<0.01 \pm 0.15 \\
0.00 \pm 0.15 \\
0.04 \pm 0.14 \\
0.01 \pm 0.14\end{array}$ & $\begin{array}{l}0.11 \pm 0.16 \\
0.12 \pm 0.16 \\
0.11 \pm 0.14 \\
0.13 \pm 0.15 \\
0.08 \pm 0.16\end{array}$ & $\begin{array}{l}-0.10 \\
-0.10 \\
-0.08 \\
-0.04 \\
-0.11\end{array}$ & $\begin{array}{l} \pm 0.16 \\
\pm 0.16 \\
\pm 0.14 \\
\pm 0.15 \\
\pm 0.16\end{array}$ & & $\begin{array}{l}5.0 \times 10^{-5} \\
4.0 \times 10^{-5} \\
1.0 \times 10^{-5}\end{array}$ \\
\hline \multicolumn{8}{|c|}{ Water Wells } \\
\hline $\begin{array}{l}O W-W R-02 \\
O W-W R-06 \\
O W-W R-16 \\
O W-W R-18\end{array}$ & $\begin{array}{l}4 \\
4 \\
6 \\
6\end{array}$ & $\begin{array}{r}0.05 \pm 0.15 \\
<0.03 \pm 0.15 \\
0.02 \pm 0.14 \\
<0.03 \pm 0.14\end{array}$ & $\begin{array}{l}0.24 \pm 0.15 \\
0.12 \pm 0.14 \\
0.06 \pm 0.16 \\
0.06 \pm 0.16\end{array}$ & $\begin{array}{l}-0.02 \\
-0.09 \\
-0.03 \\
-0.12\end{array}$ & $\begin{array}{l} \pm 0.15 \\
\pm 0.15 \\
\pm 0.16 \\
\pm 0.14\end{array}$ & . & $\begin{array}{r}5.0 \times 10^{-5} \\
<3.0 \times 10^{-5} \\
2.0 \times 10^{-5} \\
<3.0 \times 10^{-5}\end{array}$ \\
\hline & \multicolumn{7}{|c|}{ Plant Drinking Water } \\
\hline $\begin{array}{l}O W-W R-23 \\
O W-W R-28 \\
O W-W R-43\end{array}$ & $\begin{array}{l}7 \\
8 \\
1\end{array}$ & $\begin{array}{l}<0.02 \pm 0.14 \\
<0.01 \pm 0.15 \\
<0.04 \pm 0.15\end{array}$ & $\begin{array}{r}0.09 \pm 0.15 \\
0.13 \pm 0.14 \\
<0.04 \pm 0.16\end{array}$ & $\begin{array}{l}-0.11 \\
-0.10 \\
-0.04\end{array}$ & $\begin{array}{l} \pm 0.14 \\
\pm 0.14 \\
\pm 0.16\end{array}$ & & $\begin{array}{l}<2.0 \times 10^{-5} \\
<1.0 \times 10^{-5} \\
<4.0 \times 10^{-5}\end{array}$ \\
\hline & \multicolumn{7}{|c|}{ Monitor Wells } \\
\hline $\begin{array}{l}O W-W R-19 \\
O W-W R-20 \\
O W-W R-38 \\
O W-W R-39 \\
O W-W R-40\end{array}$ & $\begin{array}{r}9 \\
10 \\
7 \\
3 \\
4\end{array}$ & $\begin{array}{r}0.00 \pm 0.16 \\
<0.02 \pm 0.15 \\
<0.01 \pm 0.15 \\
<0.04 \pm 0.16 \\
0.02 \pm 0.15\end{array}$ & $\begin{array}{l}0.10 \pm 0.17 \\
0.06 \pm 0.16 \\
0.12 \pm 0.16 \\
0.04 \pm 0.15 \\
0.08 \pm 0.16\end{array}$ & $\begin{array}{l}-0.09 \\
-0.11 \\
-0.08 \\
-0.09 \\
-0.03\end{array}$ & $\begin{array}{l} \pm 0.16 \\
\pm 0.14 \\
\pm 0.15 \\
\pm 0.16 \\
\pm 0.16\end{array}$ & & $\begin{array}{r}<2.0 \times 10^{-5} \\
<1.0 \times 10^{-5} \\
<4.0 \times 10^{-5} \\
2.0 \times 10^{-5}\end{array}$ \\
\hline \multicolumn{8}{|c|}{ Industrial Waters } \\
\hline $\begin{array}{l}\text { OW-WR-09 } \\
\text { OW-WR-13 } \\
\text { OW-WR-15 } \\
\text { OW-WR-17 } \\
\text { OW-WR-25 } \\
\text { OW-WR-31 }\end{array}$ & $\begin{array}{r}11 \\
11 \\
9 \\
11 \\
10 \\
11\end{array}$ & $\begin{array}{r}0.00 \pm 0.15 \\
<0.02 \pm 0.15 \\
<0.02 \pm 0.15 \\
<0.02 \pm 0.15 \\
0.04 \pm 0.15 \\
0.01 \pm 0.15\end{array}$ & $\begin{array}{l}0.08 \pm 0.14 \\
0.08 \pm 0.14 \\
0.06 \pm 0.14 \\
0.15 \pm 0.14 \\
0.17 \pm 0.16 \\
0.30 \pm 0.15\end{array}$ & $\begin{array}{l}-0.09 \\
-0.14 \\
-0.13 \\
-0.11 \\
-0.07 \\
-0.10\end{array}$ & $\begin{array}{l} \pm 0.16 \\
\pm 0.16 \\
\pm 0.15 \\
\pm 0.15 \\
\pm 0.16 \\
\pm 0.15\end{array}$ & & $\begin{array}{l}<2.0 \times 10^{-5} \\
<2.0 \times 10^{-5} \\
<2.0 \times 10^{-5} \\
4.0 \times 10^{-5} \\
1.0 \times 10^{-5}\end{array}$ \\
\hline \multicolumn{8}{|c|}{ Control } \\
\hline $\begin{array}{l}O W-W R-33 \\
O W-W R-37\end{array}$ & $\begin{array}{r}13 \\
8\end{array}$ & $\begin{array}{r}0.01 \pm 0.15 \\
<0.01 \pm 0.14\end{array}$ & $\begin{array}{l}0.20 \pm 0.16 \\
0.06 \pm 0.15\end{array}$ & $\begin{array}{l}-0.13 \\
-0.10\end{array}$ & $\begin{array}{l} \pm 0.16 \\
\pm 0.14\end{array}$ & $\begin{array}{r}5.0 \times 10^{-4} \\
<5.0 \times 10^{-4}\end{array}$ & \\
\hline
\end{tabular}


Table X. Sumary of Plutonium-239 in Water for 1988

\begin{tabular}{|c|c|c|c|c|c|c|c|}
\hline $\begin{array}{r}\text { Samples } \\
\text { Location }\end{array}$ & $\begin{array}{l}\text { No. of } \\
\text { Samples }\end{array}$ & $\begin{array}{c}\text { Average uCl/ml } \\
\left(10^{-10}\right)\end{array}$ & $\begin{array}{l}\text { Maximum uCi/ml } \\
\left(10^{-10}\right)\end{array}$ & \multicolumn{2}{|c|}{$\begin{array}{l}\text { Minimum uCi/ml } \\
\left(10^{-10}\right) \\
\end{array}$} & \multirow[t]{2}{*}{$\begin{array}{c}\text { × OCG } \\
\text { (Average) }\end{array}$} & \multirow[t]{2}{*}{$\begin{array}{c}\text { X RCG } \\
\text { (Average) } \\
\end{array}$} \\
\hline \multicolumn{6}{|c|}{ Playas } & & \\
\hline $\begin{array}{l}O W-W R-01 \\
O W-W R-08 \\
O W-W R-24 \\
O W-W R-27 \\
O W-W R-36\end{array}$ & $\begin{array}{r}9 \\
11 \\
11 \\
6 \\
6\end{array}$ & $\begin{array}{l}0.03 \pm 0.21 \\
0.09 \pm 0.23 \\
0.05 \pm 0.22 \\
0.03 \pm 0.20 \\
0.07 \pm 0.24\end{array}$ & $\begin{array}{l}0.10 \pm 0.20 \\
0.30 \pm 0.30 \\
0.30 \pm 0.20 \\
0.20 \pm 0.20 \\
0.40 \pm 0.30\end{array}$ & $\begin{array}{r}-0.10 \\
0.00 \\
-0.10 \\
-0.10 \\
-0.20\end{array}$ & $\begin{array}{l} \pm 0.20 \\
\pm 0.20 \\
\pm 0.30 \\
\pm 0.20 \\
\pm 0.20\end{array}$ & & $\begin{array}{l}<5.0 \times 10^{-7} \\
<5.0 \times 10^{-7} \\
<5.0 \times 10^{-7} \\
<5.0 \times 10^{-7} \\
<5.0 \times 10^{-7}\end{array}$ \\
\hline \multicolumn{8}{|c|}{ Water Wells } \\
\hline $\begin{array}{l}\text { OW-WR-02 } \\
\text { OW-WR-06 } \\
\text { OW-WR-16 } \\
\text { OW-WR-18 }\end{array}$ & $\begin{array}{l}4 \\
4 \\
6 \\
6\end{array}$ & $\begin{array}{r}0.05 \pm 0.19 \\
0.10 \pm 0.23 \\
<0.03 \pm 0.22 \\
0.02 \pm 0.25\end{array}$ & $\begin{array}{l}0.20 \pm 0.10 \\
0.20 \pm 0.30 \\
0.00 \pm 0.20 \\
0.10 \pm 0.20\end{array}$ & $\begin{array}{r}-0.10 \\
0.00 \\
-0.10 \\
-0.10\end{array}$ & $\begin{array}{l} \pm 0.10 \\
\pm 0.20 \\
\pm 0.20 \\
\pm 0.20\end{array}$ & & $\begin{array}{l}<5.0 \times 10^{-7} \\
<5.0 \times 10^{-7} \\
<5.0 \times 10^{-7} \\
<5.0 \times 10^{-7}\end{array}$ \\
\hline \multicolumn{8}{|c|}{ Plant Drinking Water } \\
\hline $\begin{array}{l}O W-W R-23 \\
O W-W R-28 \\
O W-W R-43\end{array}$ & $\begin{array}{l}7 \\
8 \\
1\end{array}$ & $\begin{array}{l}<0.01 \pm 0.23 \\
<0.06 \pm 0.23 \\
<0.10 \pm 0.20\end{array}$ & $\begin{array}{r}0.20 \pm 0.20 \\
0.10 \pm 0.30 \\
<0.10 \pm 0.20\end{array}$ & $\begin{array}{l}-0.10 \\
-0.20 \\
-0.10\end{array}$ & $\begin{array}{l} \pm 0.30 \\
\pm 0.20 \\
\pm 0.20\end{array}$ & & $\begin{array}{l}<5.0 \times 10^{-7} \\
<5.0 \times 10^{-7} \\
<5.0 \times 10^{-7}\end{array}$ \\
\hline \multicolumn{8}{|c|}{ Monitor Wells } \\
\hline $\begin{array}{l}O W-W R-19 \\
O W-W R-20 \\
O W-W R-38 \\
O W-W R-39 \\
O W-W R-40\end{array}$ & $\begin{array}{r}9 \\
10 \\
7 \\
3 \\
4\end{array}$ & $\begin{array}{r}0.00 \pm 0.22 \\
0.01 \pm 0.24 \\
<0.06 \pm 0.22 \\
0.03 \pm 0.24 \\
0.03 \pm 0.20\end{array}$ & $\begin{array}{l}0.20 \pm 0.10 \\
0.20 \pm 0.20 \\
0.00 \pm 0.20 \\
0.10 \pm 0.20 \\
0.10 \pm 0.20\end{array}$ & $\begin{array}{r}-0.10 \\
-0.20 \\
-0.10 \\
0.00 \\
-0.10\end{array}$ & $\begin{array}{l} \pm 0.20 \\
\pm 0.20 \\
\pm 0.30 \\
\pm 0.30 \\
\pm 0.20\end{array}$ & & $\begin{array}{l}<5.0 \times 10^{-7} \\
<5.0 \times 10^{-7} \\
<5.0 \times 10^{-7}\end{array}$ \\
\hline \multicolumn{7}{|c|}{ Industrial Waters } & 1 \\
\hline $\begin{array}{l}O W-W R-O 9 \\
O W-W R-13 \\
O W-W R-15 \\
O W-W R-17 \\
\text { OW-WR-25 } \\
\text { OW-WR-31 }\end{array}$ & $\begin{array}{l}11 \\
11 \\
9 \\
11 \\
10 \\
11\end{array}$ & $\begin{array}{r}<0.02 \pm 0.23 \\
0.03 \pm 0.23 \\
0.02 \pm 0.23 \\
0.05 \pm 0.24 \\
0.03 \pm 0.25 \\
0.01 \pm 0.24\end{array}$ & $\begin{array}{l}0.10 \pm 0.30 \\
0.20 \pm 0.20 \\
0.10 \pm 0.20 \\
0.20 \pm 0.30 \\
0.30 \pm 0.30 \\
0.20 \pm 0.20\end{array}$ & $\begin{array}{r}-0.20 \\
-0.20 \\
-0.10 \\
0.00 \\
-0.20 \\
-0.10\end{array}$ & $\begin{array}{l} \pm 0.20 \\
\pm 0.20 \\
\pm 0.20 \\
\pm 0.20 \\
\pm 0.20 \\
\pm 0.30\end{array}$ & & $\begin{array}{l}<5.0 \times 10^{-7} \\
<5.0 \times 10^{-7} \\
<5.0 \times 10^{-7} \\
<5.0 \times 10^{-7} \\
<5.0 \times 10^{-7}\end{array}$ \\
\hline \multicolumn{8}{|c|}{ Control } \\
\hline $\begin{array}{l}O W-W R-33 \\
O W-W R-37\end{array}$ & $\begin{array}{r}13 \\
8\end{array}$ & $\begin{array}{l}<0.04 \pm 0.22 \\
<0.01 \pm 0.25\end{array}$ & $\begin{array}{l}0.10 \pm 0.10 \\
0.20 \pm 0.30\end{array}$ & $\begin{array}{l}-0.10 \\
-0.20\end{array}$ & $\begin{array}{l} \pm 0.20 \\
\pm 0.30\end{array}$ & $\begin{array}{l}<1.7 \times 10^{-5} \\
<1.7 \times 10^{-5}\end{array}$ & • \\
\hline
\end{tabular}

\section{Minimum Detectable Limit $-2 \times 10^{-11} \mathrm{uCi} / \mathrm{ml}$}

Derived Concentration Gulde (DCG) for members of the public $=3 \times 10^{-7} \mathrm{uCt} / \mathrm{mi}$

Radioactivity Concentration Guide (RCG) for controlled areas $=1 \times 10^{-4} \mathrm{uCi} / \mathrm{ml}$

Reported errors are counting errors at the $95 \%$ confidence level

Sample locations are sited on Figure 11. Sampler OW-WR-33 was located at the Bushland Agriculture Research Station (Figure 1)

Water Sample locations are described in Table A-VI 
Table XI. Summary of Uranium-234 in Water for 1988

\begin{tabular}{|c|c|c|c|c|c|c|}
\hline $\begin{array}{l}\text { Samples } \\
\text { Location }\end{array}$ & $\begin{array}{l}\text { No. of } \\
\text { Samples }\end{array}$ & $\begin{array}{l}\text { Average } u C i / m l \\
\left(10^{-9}\right)\end{array}$ & $\begin{array}{l}\text { Maximum uCi/ml } \\
\left(10^{-9}\right) \\
\end{array}$ & $\begin{array}{l}\text { Minimum uCi/ml } \\
\left(10^{-9}\right)\end{array}$ & $\begin{array}{c}\text { X DCG } \\
\text { (Averaqe) } \\
\end{array}$ & $\begin{array}{c}\text { X RCG } \\
\text { (Average) }\end{array}$ \\
\hline \multicolumn{7}{|c|}{ Playas } \\
\hline $\begin{array}{l}O W-W R-01 \\
O W-W R-08 \\
O W-W R-24 \\
O W-W R-27 \\
O W-W R-36\end{array}$ & $\begin{array}{r}9 \\
11 \\
11 \\
6 \\
6\end{array}$ & $\begin{array}{l}0.76 \pm 0.23 \\
2.08 \pm 0.38 \\
2.31 \pm 0.39 \\
0.87 \pm 0.28 \\
0.68 \pm 0.24\end{array}$ & $\begin{array}{l}1.40 \pm 0.30 \\
4.50 \pm 0.60 \\
5.10 \pm 0.60 \\
4.70 \pm 0.60 \\
3.40 \pm 0.50\end{array}$ & $\begin{array}{l}0.30 \pm 0.20 \\
0.10 \pm 0.10 \\
0.10 \pm 0.20 \\
0.00 \pm 0.20 \\
0.00 \pm 0.10\end{array}$ & & $\begin{array}{l}1.9 \times 10^{-2} \\
5.2 \times 10^{-2} \\
5.8 \times 10^{-2} \\
2.2 \times 10^{-2} \\
1.7 \times 10^{-2}\end{array}$ \\
\hline \multicolumn{7}{|c|}{ Water Wells } \\
\hline $\begin{array}{l}\text { OW-WR-02 } \\
\text { OW-WR-06 } \\
\text { OW-WR-16 } \\
\text { OW-WR-18 }\end{array}$ & $\begin{array}{l}4 \\
4 \\
6 \\
6\end{array}$ & $\begin{array}{l}4.22 \pm 0.53 \\
3.95 \pm 0.48 \\
3.30 \pm 0.43 \\
3.67 \pm 0.51\end{array}$ & $\begin{array}{l}4.80 \pm 0.60 \\
5.00 \pm 0.60 \\
6.10 \pm 0.70 \\
6.00 \pm 0.80\end{array}$ & $\begin{array}{l}3.60 \pm 0.40 \\
3.50 \pm 0.40 \\
0.10 \pm 0.20 \\
2.20 \pm 0.30\end{array}$ & . & $\begin{array}{l}1.0 \times 10^{-2} \\
9.9 \times 10^{-2} \\
8.2 \times 10^{-2} \\
9.2 \times 10^{-2}\end{array}$ \\
\hline \multicolumn{7}{|c|}{ Plant Drinking Water } \\
\hline $\begin{array}{l}O W-W R-23 \\
O W-W R-28 \\
O W-W R-43\end{array}$ & $\begin{array}{l}7 \\
8 \\
1\end{array}$ & $\begin{array}{l}4.10 \pm 0.47 \\
3.82 \pm 0.50 \\
4.40 \pm 0.70\end{array}$ & $\begin{array}{l}4.90 \pm 0.60 \\
5.20 \pm 0.60 \\
4.40 \pm 0.70\end{array}$ & $\begin{array}{l}3.30 \pm 0.40 \\
1.20 \pm 0.20 \\
4.40 \pm 0.70\end{array}$ & & $\begin{array}{l}1.0 \times 10^{-2} \\
9.6 \times 10^{-2} \\
1.1 \times 10^{-2}\end{array}$ \\
\hline \multicolumn{7}{|c|}{ Monitor Wels } \\
\hline $\begin{array}{l}O W-W R-19 \\
O W-W R-20 \\
O W-W R-38 \\
O W-W R-39 \\
O W-W R-40\end{array}$ & $\begin{array}{r}9 \\
10 \\
7 \\
3 \\
4\end{array}$ & $\begin{array}{l}0.16 \pm 0.14 \\
0.22 \pm 0.16 \\
2.09 \pm 0.37 \\
0.83 \pm 0.26 \\
0.25 \pm 0.16\end{array}$ & $\begin{array}{l}0.60 \pm 0.20 \\
0.70 \pm 0.20 \\
2.80 \pm 0.40 \\
2.00 \pm 0.40 \\
0.80 \pm 0.20\end{array}$ & $\begin{array}{l}0.00 \pm 0.10 \\
0.00 \pm 0.10 \\
0.80 \pm 0.20 \\
0.00 \pm 0.10 \\
0.00 \pm 0.10\end{array}$ & & $\begin{array}{l}4.0 \times 10^{-2} \\
5.5 \times 10^{-2} \\
5.2 \times 10^{-2} \\
2.1 \times 10^{-2} \\
6.3 \times 10^{-2}\end{array}$ \\
\hline \multicolumn{5}{|c|}{ Industrial Waters } & & ' \\
\hline $\begin{array}{l}\text { OW-WR-09 } \\
\text { OW-WR-13 } \\
\text { OW-WR-15 } \\
\text { OW-WR-17 } \\
\text { OW-WR-25 } \\
\text { OW-WR-31 }\end{array}$ & $\begin{array}{r}11 \\
11 \\
9 \\
11 \\
10 \\
11\end{array}$ & $\begin{array}{l}4.22 \pm 0.58 \\
3.79 \pm 0.50 \\
3.93 \pm 0.51 \\
4.35 \pm 0.57 \\
3.68 \pm 0.49 \\
3.37 \pm 0.48\end{array}$ & $\begin{array}{l}7.20 \pm 1.00 \\
5.30 \pm 0.70 \\
6.10 \pm 0.60 \\
7.20 \pm 0.60 \\
5.80 \pm 0.70 \\
5.50 \pm 0.60\end{array}$ & $\begin{array}{l}1.70 \pm 0.40 \\
0.40 \pm 0.10 \\
2.00 \pm 0.30 \\
2.20 \pm 0.50 \\
0.00 \pm 0.10 \\
0.50 \pm 0.20\end{array}$ & & $\begin{array}{l}1.0 \times 10^{-2} \\
9.5 \times 10^{-2} \\
9.8 \times 10^{-2} \\
1.1 \times 10^{-2} \\
9.2 \times 10^{-2} \\
8.4 \times 10^{-2}\end{array}$ \\
\hline
\end{tabular}

$\begin{array}{lrrrrr}\text { OW-WR-33 } & 13 & 2.64 \pm 0.43 & 3.40 \pm 0.30 & 0.10 \pm 0.20 & 0.53 \\ \text { OW-WR-37 } & 8 & 2.81 \pm 0.53 & 4.70 \pm 0.70 & 1.10 \pm 0.30 & 0.56\end{array}$

Minimum Datectable Limit $=3 \times 10^{-10} \mathrm{uCl} / \mathrm{ml}$

Derived Concentration Gulde (DCG) for members of the public $=5 \times 10^{-7} \mathrm{uCl} / \mathrm{ml}$

Radioactivity Concentration Guide (RCG) for controlled areas $=4 \times 10^{-6} \mathrm{uCj} / \mathrm{ml}$

Reported errors are counting errors at the $95 \%$ confidence level
Sample locations are sited on Figure 11 . Sampler OW-WR-33 was located at the Bushland Agricultural Research Station (Figure 1 )

Sample locations are sited on Figure 11. Sampler 
Table XII. Summary of Uranium-238 in Water for 1988

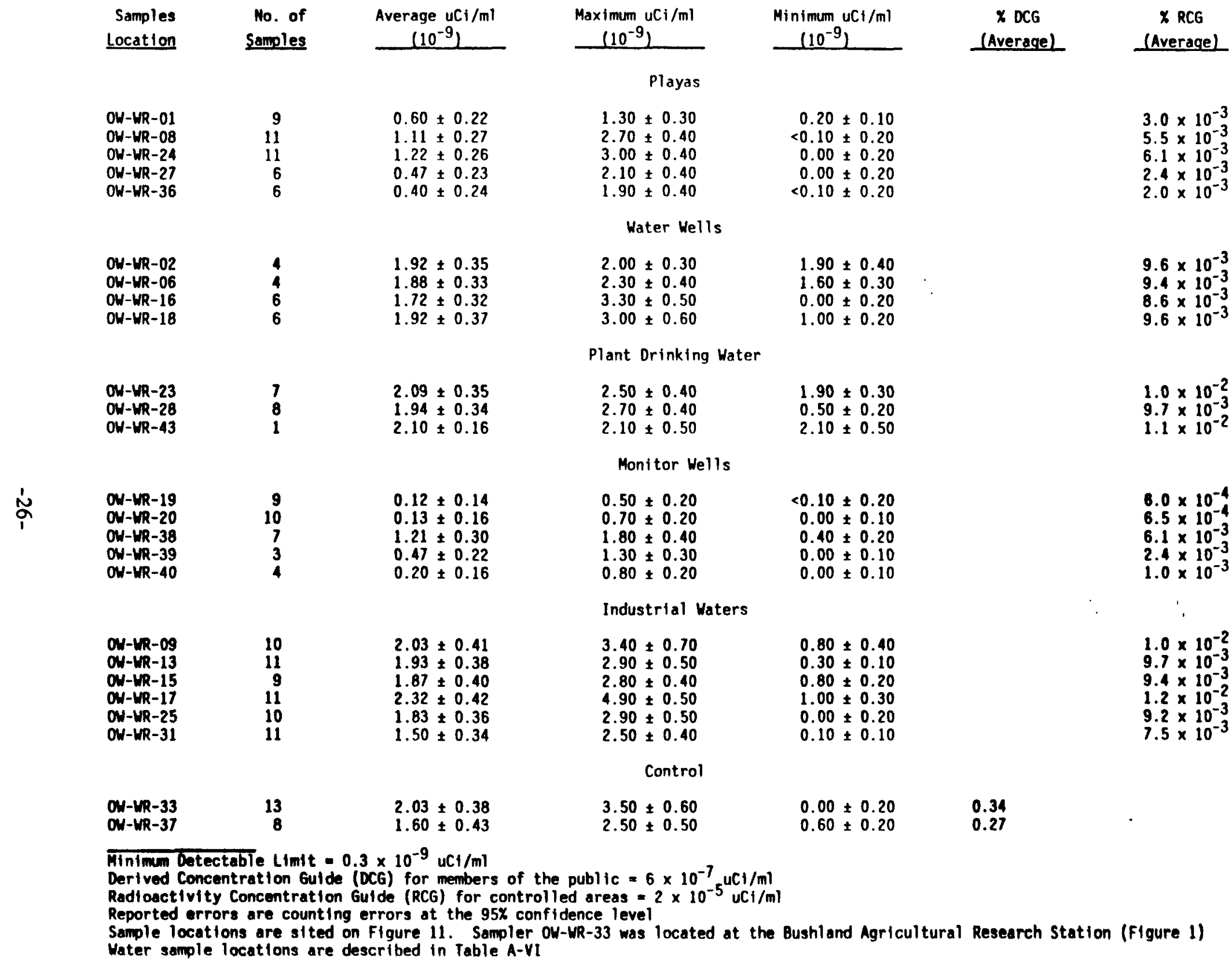

$x$ DCG

x RCG 
Table XIII. Summary of Radium-226 in Water for 1988

\begin{tabular}{|c|c|c|c|c|c|c|}
\hline $\begin{array}{r}\text { Samples } \\
\text { Location } \\
\end{array}$ & $\begin{array}{r}\text { No. of } \\
\text { Samples } \\
\end{array}$ & $\begin{array}{l}\text { Average } \mathrm{uCl} / \mathrm{m}] \\
\left(10^{-9}\right) \\
\end{array}$ & $\begin{array}{l}\text { Maximum uCi/ml } \\
\left(10^{-9}\right)\end{array}$ & $\begin{array}{c}\text { Minimum } u C i / m l \\
\left(10^{-9}\right) \\
\end{array}$ & $\begin{array}{c}\text { X DCG } \\
\text { (Averaqe) }\end{array}$ & $\begin{array}{c}\times \text { RCG } \\
\text { (Average) }\end{array}$ \\
\hline \multicolumn{7}{|c|}{ Water Wells } \\
\hline $\begin{array}{l}O W-W R-02 \\
O W-W R-06 \\
O W-W R-16 \\
O W-W R-18\end{array}$ & $\begin{array}{l}3 \\
3 \\
3 \\
3\end{array}$ & $\begin{array}{l}0.27 \pm 0.20 \\
0.17 \pm 0.20 \\
0.00 \pm 0.25 \\
0.23 \pm 0.25\end{array}$ & $\begin{array}{l}0.40 \pm 0.20 \\
0.30 \pm 0.20 \\
0.30 \pm 0.30 \\
0.60 \pm 0.30\end{array}$ & $\begin{array}{r}0.20 \pm 0.20 \\
0.00 \pm 0.20 \\
-0.30 \pm 0.30 \\
-0.20 \pm 0.30\end{array}$ & & $\begin{array}{l}6.7 \pm 10^{-2} \\
4.2 \pm 10^{-2} \\
1.0 \pm 10^{-2} \\
5.7 \pm 10^{-2}\end{array}$ \\
\hline \multicolumn{7}{|c|}{ Control } \\
\hline OW-WR-33 & 3 & $0.40 \pm 0.31$ & $1.00 \pm 0.30$ & $-0.10 \pm 0.20$ & $4.0 \pm 10^{-1}$ & \\
\hline
\end{tabular}

Minimum Detectable LImit $=6 \times 10^{-10} \mathrm{uCl} / \mathrm{ml}$

Derived Concentration Gutde (DCG) for members of the public $=1 \times 10^{-7} \mathrm{uCi} / \mathrm{ml}$

Radioactivity Concentration Gutde (RCG) for controlled areas $=4 \times 10^{-7} \mathrm{uCi} / \mathrm{ml}$

Reported errors are counting errors at the $95 \%$ confidence level

Sample locations are sited on Figure 11. Sampler OW-WR-33 was located at the Bushland Agriculture Research Station (Figure 1)

Hater sample locattons ore described in Table A-VI

40 CFR 141 lists maximm contaminate level for combined Radium-226 and -228 as $5 \times 10^{-9} \mathrm{uCi} / \mathrm{ml}$ 
Table XIV. Summary of Radium-228 in Water for 1988

\begin{tabular}{|c|c|c|c|c|c|c|}
\hline $\begin{array}{r}\text { Samples } \\
\text { Location }\end{array}$ & $\begin{array}{l}\text { No. of } \\
\text { Samples }\end{array}$ & $\begin{array}{c}\text { Average } u C i / m) \\
\left(10^{-9}\right)\end{array}$ & $\begin{array}{l}\text { Maximum uCi/ml } \\
\left(10^{-9}\right)\end{array}$ & $\begin{array}{l}\text { Minimum } u C i / m 1 \\
\left(10^{-9}\right) \\
\end{array}$ & $\begin{array}{c}\text { X DCG } \\
\text { (Average) }\end{array}$ & $\begin{array}{c}\text { X RCG } \\
\text { (Average) }\end{array}$ \\
\hline \multicolumn{7}{|c|}{ Water Wells } \\
\hline $\begin{array}{l}O W-W R-02 \\
O W-W R-06 \\
O W-W R-16 \\
O W-W R-18\end{array}$ & $\begin{array}{l}3 \\
3 \\
3 \\
3\end{array}$ & $\begin{array}{l}0.20 \pm 1.01 \\
0.17 \pm 1.01 \\
0.57 \pm 1.09 \\
0.43 \pm 0.84\end{array}$ & $\begin{array}{l}0.40 \pm 1.10 \\
0.40 \pm 1.10 \\
0.90 \pm 0.70 \\
0.50 \pm 0.90\end{array}$ & $\begin{array}{l}0.00 \pm 0.80 \\
0.00 \pm 0.80 \\
0.10 \pm 1.50 \\
0.40 \pm 0.70\end{array}$ & & $\begin{array}{l}2.5 \pm 10^{-2} \\
2.1 \pm 12^{-2} \\
7.1 \pm 10^{-2} \\
5.4 \pm 10^{-2}\end{array}$ \\
\hline \multicolumn{7}{|c|}{ Control } \\
\hline OW-WR-33 & 3 & $0.40 \pm 0.84$ & $1.20 \pm 1.00$ & $-0.10 \pm 0.80$ & $4.0 \pm 10^{-1}$ & \\
\hline
\end{tabular}

Minimum Detectable Limit $=1 \times 10^{-9} \mathrm{uCl} / \mathrm{ml}$

Derived Concentration Guide (DCG) for members of the public $=1 \times 10^{-7} \mathrm{uCi} / \mathrm{ml}$

Radioactivity Concentration Gulde (RCG) for controlled areas $=8 \times 10^{-7} \mathrm{uCi} / \mathrm{m}$

Reported errors are counting errors at the $95 \%$ confidence level

Sample locations are sited on Figure 11 . Sample site OW-WR-33 was located at the Bushland Agriculture Research Statlon (Figure 1$)$

i 40 CFR 141 lists maximm contaminate level for combined Radium-226 and -228 as $5 \times 10^{-9} \mathrm{uCi} / \mathrm{ml}$ 
Table XV. Summary of Dissolved Alpha in Water for 1988

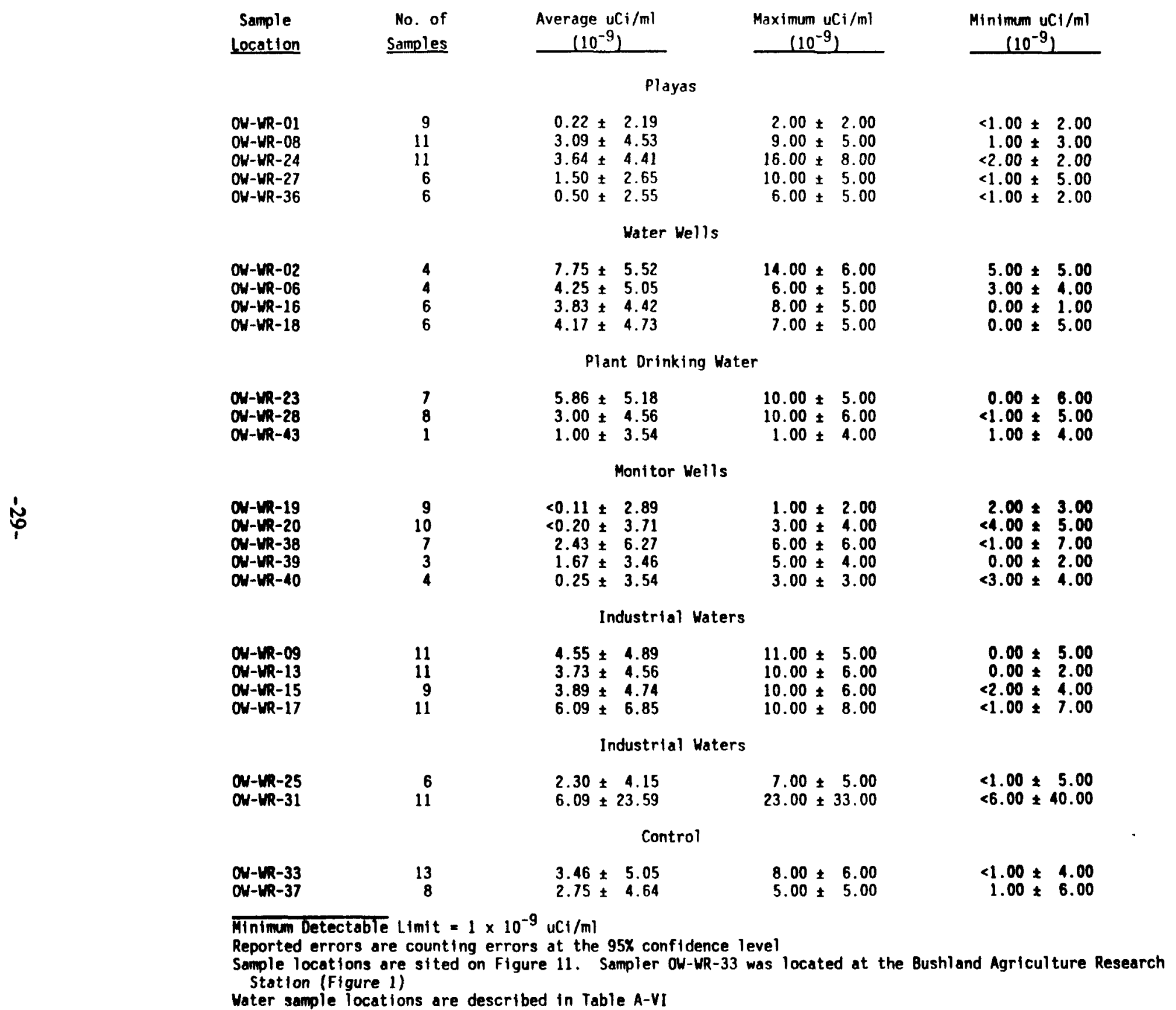


Table XVI. Summary of Suspended Alpha in Water for 1988

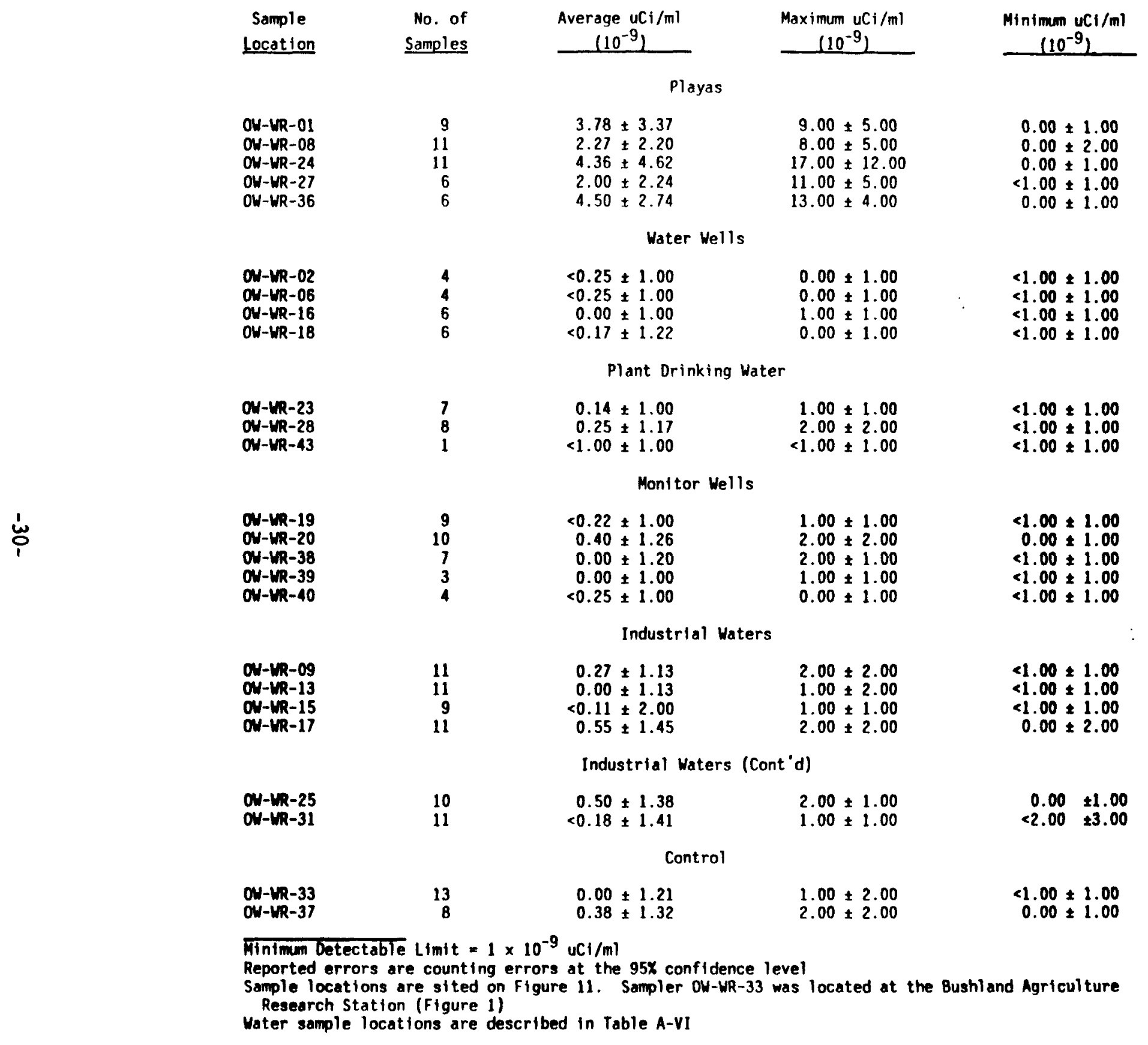


Table XVII. Summary of Dissolved Beta in Water for 1988

\begin{tabular}{|c|c|c|c|c|c|c|}
\hline & $\begin{array}{c}\text { Sample } \\
\text { Location }\end{array}$ & $\begin{array}{l}\text { No. of } \\
\text { Samples }\end{array}$ & $\begin{array}{l}\text { Average uCi/ml } \\
\left(10^{-9}\right)^{-}\end{array}$ & $\begin{array}{l}\text { Maximum } u C i / m] \\
\left(10^{-9}\right)\end{array}$ & $\begin{array}{r}\text { Minimum y } \\
\left(10^{-9}\right. \\
\end{array}$ & $\begin{array}{l}c_{1} / m 1 \\
2\end{array}$ \\
\hline & & & Pla & & & \\
\hline & $\begin{array}{l}O W-W R-01 \\
O W-W R-08 \\
O W-W R-24 \\
O W-W R-27 \\
O W-W R-36\end{array}$ & $\begin{array}{r}9 \\
11 \\
11 \\
6 \\
6\end{array}$ & $\begin{array}{l}10.44 \pm 2.81 \\
14.91 \pm 3.21 \\
13.64 \pm 3.21 \\
11.17 \pm 3.00 \\
15.50 \pm 3.19\end{array}$ & $\begin{array}{l}16.00 \pm 3.00 \\
23.00 \pm 4.00 \\
22.00 \pm 4.00 \\
14.00 \pm 3.00 \\
26.00 \pm 4.00\end{array}$ & $\begin{array}{l}2.00 \pm \\
6.00 \pm \\
7.00 \pm \\
5.00 \pm \\
9.00 \pm\end{array}$ & $\begin{array}{l}2.00 \\
3.00 \\
3.00 \\
3.00 \\
3.00\end{array}$ \\
\hline & & & Water & & & \\
\hline & $\begin{array}{l}O W-W R-02 \\
O W-W R-06 \\
O W-W R-16 \\
O W-W R-18\end{array}$ & $\begin{array}{l}4 \\
4 \\
6 \\
6\end{array}$ & $\begin{array}{l}8.25 \pm 3.00 \\
7.50 \pm 3.00 \\
7.50 \pm 2.86 \\
7.17 \pm 3.00\end{array}$ & $\begin{array}{r}9.00 \pm 3.00 \\
8.00 \pm 3.00 \\
10.00 \pm 3.00 \\
10.00 \pm 3.00\end{array}$ & $\begin{array}{l}7.00 \pm \\
7.00 \pm \\
3.00 \pm \\
5.00 \pm\end{array}$ & $\begin{array}{l}3.00 \\
3.00 \\
2.00 \\
3.00\end{array}$ \\
\hline & & & Plant Drini & & & \\
\hline & $\begin{array}{l}O W-W R-23 \\
O W-W R-28 \\
O W-W R-43\end{array}$ & $\begin{array}{l}7 \\
8 \\
1\end{array}$ & $\begin{array}{l}8.71 \pm 3.00 \\
8.63 \pm 3.00 \\
8.00 \pm 3.00\end{array}$ & $\begin{array}{r}15.00 \pm 3.00 \\
15.00 \pm 3.00 \\
8.00 \pm 3.00\end{array}$ & $\begin{array}{l}6.00 \pm \\
5.00 \pm \\
8.00 \pm\end{array}$ & $\begin{array}{l}3.00 \\
3.00 \\
3.00\end{array}$ \\
\hline & & & Monttor & & & \\
\hline & $\begin{array}{l}O W-W R-19 \\
\text { ON-WR-20 } \\
\text { OW-WR-38 } \\
\text { ON-WR-39 } \\
\text { OW-WR-40 }\end{array}$ & $\begin{array}{r}9 \\
10 \\
7 \\
3 \\
4\end{array}$ & $\begin{array}{l}3.56 \pm 2.71 \\
7.30 \pm 3.00 \\
6.29 \pm 3.82 \\
5.33 \pm 3.00 \\
6.00 \pm 3.00\end{array}$ & $\begin{array}{r}9.00 \pm 3.00 \\
14.00 \pm 3.00 \\
11.00 \pm 5.00 \\
6.00 \pm 3.00 \\
7.00 \pm 3.00\end{array}$ & $\begin{array}{l}1.00= \\
4.00= \\
1.00= \\
4.00= \\
5.00 \pm\end{array}$ & $\begin{array}{l}2.00 \\
3.00 \\
5.00 \\
3.00 \\
3.00\end{array}$ \\
\hline & & & Industria & & & \\
\hline & $\begin{array}{l}O N-W R-O 9 \\
O N-W R-13 \\
O N-W_{R}-15 \\
O W-W R-17\end{array}$ & $\begin{array}{l}11 \\
11 \\
9 \\
11\end{array}$ & $\begin{array}{r}8.09 \pm 3.00 \\
12.55 \pm 3.16 \\
7.89 \pm 3.00 \\
10.82 \pm 3.75\end{array}$ & $\begin{array}{l}16.00 \pm 3.00 \\
73.00 \pm 5.00 \\
13.00 \pm 3.00 \\
17.00 \pm 4.00\end{array}$ & $\begin{array}{l}4.00 \pm \\
3.00 \pm \\
6.00 \pm \\
6.00 \pm\end{array}$ & $\begin{array}{l}3.00 \\
2.00 \\
3.00 \\
3.00\end{array}$ \\
\hline & & & Industrial Wa & (t'd) & & \\
\hline & $\begin{array}{l}O W-W R-25 \\
O W-W R-31\end{array}$ & $\begin{array}{l}10 \\
11\end{array}$ & $\begin{array}{r}7.70 \pm 3.00 \\
11.00 \pm 19.95\end{array}$ & $\begin{array}{r}19.00 \pm 3.00 \\
34.00 \pm 27.00\end{array}$ & $\begin{array}{r}3.00 \pm \\
<2.00 \pm\end{array}$ & $\begin{array}{r}3.00 \\
44.00\end{array}$ \\
\hline & & & Cont & & & \\
\hline & $\begin{array}{l}\text { ON-WR-33 } \\
\text { ON-WR-37 }\end{array}$ & $\begin{array}{r}13 \\
8\end{array}$ & $\begin{array}{l}7.62 \pm 3.00 \\
8.00 \pm 3.00\end{array}$ & $\begin{array}{l}15.00 \pm 3.00 \\
16.00 \pm 3.00\end{array}$ & $\begin{array}{l}4.00 \pm \\
5.00 \pm\end{array}$ & $\begin{array}{l}3.00 \\
3.00\end{array}$ \\
\hline & $\begin{array}{l}\text { Winimum D } \\
\text { Reported } \\
\text { Sample lo } \\
\text { Researc } \\
\text { Water sam }\end{array}$ & Limit & $\begin{array}{l}\text { uCi/ml } \\
\text { at the } 95 \% \text { conf } \\
\text { 11. Sampler } 0 \\
\text { d in Table } A-v I\end{array}$ & ted at & 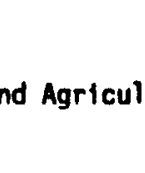 & \\
\hline
\end{tabular}


Table XVIII. Summary of Suspended Beta in Water for 1988

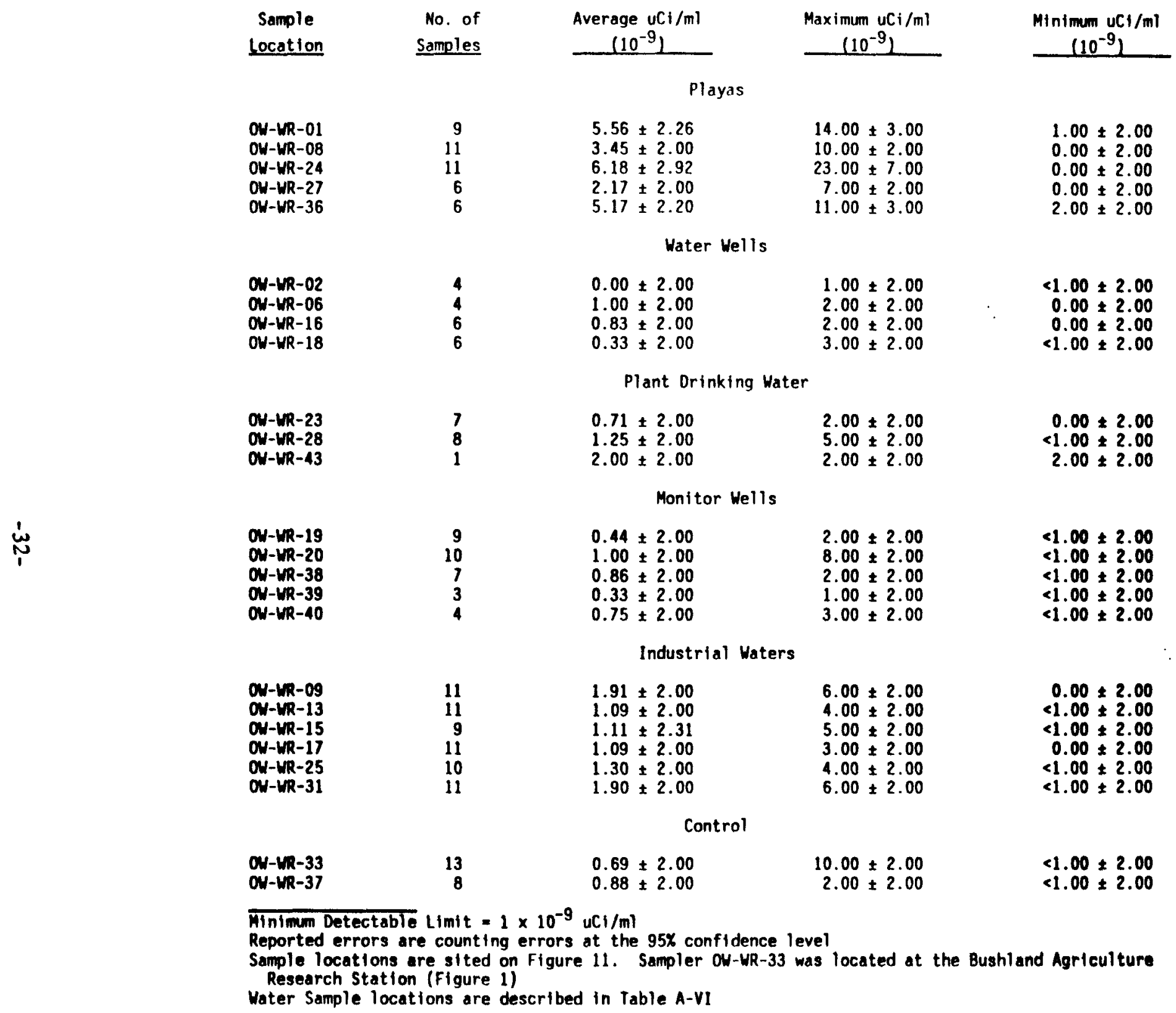


Table XIX. Summary of Beryllium in Soil for 1988

\begin{tabular}{|c|c|c|c|c|}
\hline $\begin{array}{c}\text { Sample } \\
\text { Location }\end{array}$ & $\begin{array}{l}\text { No. of } \\
\text { Samples }\end{array}$ & Averaqe ug/g & Maximum ug/g & Minimum ug/g \\
\hline $\begin{array}{l}F A-S S-01 \\
F B-S S-01 \\
F B-S S-02 \\
F B-S S-03 \\
F B-S S-04 \\
F B-S S-05 \\
F B-S S-06 \\
F B-S S-07 \\
F B-S S-08 \\
F B-S S-09 \\
F C-S S-01 \\
F C-S S-02 \\
F C-S S-03 \\
F C-S S-04 \\
F C-S S-05 \\
O S-S S-32\end{array}$ & $\begin{array}{l}12 \\
12 \\
12 \\
12 \\
12 \\
12 \\
12 \\
12 \\
12 \\
12 \\
12 \\
12 \\
12 \\
12 \\
12 \\
12\end{array}$ & $\begin{array}{l}1.19 \\
1.14 \\
1.29 \\
1.15 \\
1.14 \\
1.32 \\
1.30 \\
1.22 \\
1.27 \\
1.14 \\
1.04 \\
0.97 \\
0.94 \\
1.06 \\
1.05 \\
0.93\end{array}$ & $\begin{array}{l}1.60 \\
1.40 \\
1.70 \\
1.60 \\
1.50 \\
2.60 \\
2.40 \\
1.50 \\
1.40 \\
1.50 \\
1.20 \\
1.10 \\
1.10 \\
1.20 \\
1.30 \\
1.30\end{array}$ & $\begin{array}{l}0.80 \\
0.80 \\
1.00 \\
0.70 \\
0.80 \\
0.80 \\
0.90 \\
0.90 \\
1.10 \\
0.50 \\
0.70 \\
0.80 \\
0.60 \\
0.70 \\
0.80 \\
0.50\end{array}$ \\
\hline
\end{tabular}

Minimum Detectable Limit $=0.05 \mathrm{ug} / \mathrm{g}$ of dry soil

Sample locations are sited on Figures 8, 9, and 10. Sampler site BU-SS-01 was located at the Bushiand Agricultural Research Station (Figure 1) 
Table XX. Summary of Plutonium-239 in Soil for 1988

\begin{tabular}{|c|c|c|c|c|c|c|c|}
\hline & $\begin{array}{c}\text { Sample } \\
\text { Location }\end{array}$ & $\begin{array}{r}\text { No. of } \\
\text { Samples } \\
\end{array}$ & $\begin{array}{r}\text { Averag } \\
\quad 10 \\
\end{array}$ & $\begin{array}{l}\text { ge } u C i / g \\
\left.0^{-6}\right)\end{array}$ & $\begin{array}{r}\text { Maxim } \\
\quad 11 \\
\end{array}$ & $\begin{array}{l}u m u C i / g \\
\left.0^{-6}\right)\end{array}$ & $\begin{array}{c}\text { Minimm uCi/g } \\
\left(10^{-6}\right)\end{array}$ \\
\hline & & & & Plan & & & \\
\hline & & & & off & & & \\
\hline & $\begin{array}{l}\text { OS-SS-01 } \\
\text { OS-SS-02 } \\
\text { OS-SS-03 } \\
\text { OS-SS-04 } \\
\text { OS-SS-05 } \\
\text { OS-SS-06 } \\
\text { OS-SS-07 } \\
\text { OS-SS-08 } \\
\text { OS-SS-09 } \\
\text { OS-SS-10 } \\
\text { OS-SS-11 } \\
\text { OS-SS-12 } \\
\text { OS-SS-13 } \\
\text { OS-SS-14 } \\
\text { OS-SS-15 } \\
\text { OS-SS-16 } \\
\text { OS-SS-17 } \\
\text { OS-SS-18 } \\
\text { OS-SS-19 } \\
\text { OS-SS-20 } \\
\text { OS-SS-21 } \\
\text { OS-SS-22 } \\
\text { OS-SS-23 } \\
\text { OS-SS-24 } \\
\text { OS-SS-25 }\end{array}$ & $\begin{array}{l}3 \\
3 \\
3 \\
3 \\
3 \\
3 \\
3 \\
3 \\
3 \\
3 \\
3 \\
3 \\
3 \\
3 \\
3 \\
3 \\
3 \\
3 \\
3 \\
3 \\
3 \\
3 \\
3 \\
3 \\
3\end{array}$ & $\begin{array}{l}0.00 \\
0.00 \\
0.00 \\
0.01 \\
0.01 \\
0.00 \\
0.01 \\
0.01 \\
0.01 \\
0.00 \\
0.00 \\
0.00 \\
0.01 \\
0.01 \\
0.00 \\
0.00 \\
0.00 \\
0.00 \\
0.01 \\
0.00 \\
0.01 \\
0.01 \\
0.02 \\
0.01 \\
0.01\end{array}$ & $\begin{array}{l} \pm 0.02 \\
\pm 0.02 \\
\pm 0.02 \\
\pm 0.02 \\
\pm 0.02 \\
\pm 0.02 \\
\pm 0.02 \\
\pm 0.02 \\
\pm 0.02 \\
\pm 0.02 \\
\pm 0.02 \\
\pm 0.02 \\
\pm 0.02 \\
\pm 0.02 \\
\pm 0.02 \\
\pm 0.02 \\
\pm 0.02 \\
\pm 0.02 \\
\pm 0.02 \\
\pm 0.02 \\
\pm 0.02 \\
\pm 0.02 \\
\pm 0.02 \\
\pm 0.02 \\
\pm 0.02\end{array}$ & $\begin{array}{l}0.00 \\
0.01 \\
0.00 \\
0.02 \\
0.02 \\
0.00 \\
0.02 \\
0.02 \\
0.02 \\
0.01 \\
0.00 \\
0.00 \\
0.03 \\
0.01 \\
0.01 \\
0.00 \\
0.00 \\
0.00 \\
0.02 \\
0.00 \\
0.02 \\
0.03 \\
0.03 \\
0.01 \\
0.02\end{array}$ & $\begin{array}{l} \pm 0.02 \\
\pm 0.02 \\
\pm 0.02 \\
\pm 0.02 \\
\pm 0.02 \\
\pm 0.02 \\
\pm 0.02 \\
\pm 0.02 \\
\pm 0.02 \\
\pm 0.02 \\
\pm 0.02 \\
\pm 0.02 \\
\pm 0.03 \\
\pm 0.02 \\
\pm 0.02 \\
\pm 0.02 \\
\pm 0.02 \\
\pm 0.02 \\
\pm 0.03 \\
\pm 0.02 \\
\pm 0.03 \\
\pm 0.03 \\
\pm 0.03 \\
\pm 0.02 \\
\pm 0.02\end{array}$ & $\begin{array}{r}0.00 \pm 0.02 \\
0.00 \pm 0.01 \\
0.00 \pm 0.02 \\
0.00 \pm 0.03 \\
0.01 \pm 0.02 \\
0.00 \pm 0.02 \\
0.00 \pm 0.02 \\
0.01 \pm 0.02 \\
<0.01 \pm 0.02 \\
0.00 \pm 0.01 \\
0.00 \pm 0.02 \\
0.00 \pm 0.02 \\
0.00 \pm 0.02 \\
0.00 \pm 0.02 \\
0.00 \pm 0.02 \\
0.00 \pm 0.02 \\
<0.01 \pm 0.02 \\
0.00 \pm 0.02 \\
0.00 \pm 0.02 \\
0.00 \pm 0.02 \\
0.00 \pm 0.02 \\
0.00 \pm 0.02 \\
0.01 \pm 0.02 \\
0.00 \pm 0.02 \\
0.00 \pm 0.01\end{array}$ \\
\hline
\end{tabular}


Table XX. Summary of Plutonium-239 in Soil for 1988 (Cont'd)

\begin{tabular}{|c|c|c|c|c|}
\hline $\begin{array}{c}\text { Sample } \\
\text { Location }\end{array}$ & $\begin{array}{r}\text { No. of } \\
\text { Samples } \\
\end{array}$ & $\begin{array}{c}\text { Average } \mathrm{uCi} / \mathrm{g} \\
\left(10^{-6}\right)\end{array}$ & $\begin{array}{c}\text { Maximum uCi/g } \\
\left(10^{-6}\right)\end{array}$ & $\begin{array}{c}\text { Minimum } u C t / g \\
\left(10^{-6}\right)\end{array}$ \\
\hline \multicolumn{5}{|c|}{ Off Site (Cont $\left.{ }^{\prime} d\right)$} \\
\hline $\begin{array}{l}\text { OS-SS-26 } \\
\text { OS-SS-27 } \\
\text { OS-SS-28 } \\
\text { OS-SS-29 } \\
\text { OS-SS-30 } \\
\text { OS-SS-31 }\end{array}$ & $\begin{array}{l}3 \\
3 \\
3 \\
3 \\
3 \\
3\end{array}$ & 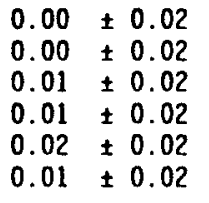 & $\begin{array}{ll}0.02 & \pm 0.02 \\
0.01 & \pm 0.02 \\
0.01 & \pm 0.03 \\
0.04 & \pm 0.02 \\
0.03 & \pm 0.03 \\
0.02 & \pm 0.02\end{array}$ & $\begin{aligned}<0.01 & \pm 0.02 \\
0.00 & \pm 0.02 \\
0.01 & \pm 0.03 \\
0.00 & \pm 0.02 \\
0.01 & \pm 0.02 \\
0.01 & \pm 0.02\end{aligned}$ \\
\hline \multicolumn{5}{|c|}{ Control } \\
\hline OS-5S-32 & 13 & $0.01 \pm 0.02$ & $0.03 \pm 0.02$ & $0.00 \pm 0.02$ \\
\hline
\end{tabular}

Minimum Detectable Limit $=2 \times 10^{-8} \mathrm{uCi} / \mathrm{g}$

Reported errors are counting errors at the $95 \%$ confidence level

Activity is per gram of dry soil

ample locations are sited on Figures 7,8,9. and 10. Sample site BU-SS-01 was located at the Bushland Agriculture Research Station (Figure 1) 
Table XX1. Sunmary of Uranium-234 in Soil for 1988

\begin{tabular}{l} 
Sample \\
Location \\
\hline \\
BG-SS-01 \\
BG-SS-02 \\
BG-SS-03 \\
BG-SS-04 \\
FA-SS-01 \\
FB-SS-01 \\
FB-SS-02 \\
FB-SS-03 \\
FB-SS-04 \\
FB-SS-05 \\
FB-SS-06 \\
FB-SSS-07 \\
FB-SS-08 \\
FB-SS-09 \\
FC-SS 01 \\
FC-SS-02 \\
FC-SS-03 \\
FC-SS-04 \\
FC-SS-05
\end{tabular}

No. of

Average $\mathrm{UCi} / \mathrm{g}$

Samples

$110^{-7}$

Maximum $u C i / g$

Minimum $u C l / g$

Plant Site

$6.33 \pm 1.71$

$6.00 \pm 1.58$

$.17 \pm 1.50$

$7.75 \pm 1.73$

$16.50 \pm 2.35$

$48.25 \pm 4.80$

$18.08 \pm 2.42$

$16.25 \pm 2.25$
$14.92 \pm 2.22$

$21.33+2.60$

$14.50 \pm 2.22$

$12.17 \pm 2.06$

$12.17 \pm 2.06$
$10.17 \pm 1.78$

$10.17 \pm 1.78$
$21.67+2.99$

$21.67 \pm 2.99$
$11.42 \pm 1.87$

$11.42 \pm 1.87$
$9.08 \pm 1.87$

$16.50 \pm 3.39$

$13.83 \pm 2.24$

$52.58 \pm 3.46$

$10.00 \pm 2.00$

$7.00 \pm 1.00$

$10.00 \pm 4.00$

$13.00 \pm 2.00$

$49.00 \pm 3.00$

$110.00 \pm 10.00$
$51.00 \pm 3.00$

$51.00 \pm 3.00$
$26.00 \pm 3.00$

$38.00 \pm 3.00$

$47.00 \pm 3.00$

$36.00 \pm 3.00$

$23.00 \pm 2.00$

$12.00 \pm 1.00$

$49.00 \pm 3.00$

$20.00 \pm 2.00$

$13.00 \pm 2.00$

$110.00 \pm 10.00$

$17.00 \pm 2.00$

off-site

OS-SS-01
OS-SS-02
OS-SS-03
OS-SS-04
OS-SS-05
OS-SS-06
OS-SS-07
OS-SS-08
OS-SS-09
OS-SS-10
OS-SS-11
OS-SS-12
OS-SS-13
OS-SS-14
OS-SS-15
OS-SS-16
OS-SS-17
OS-SS-18
OS-SS-19
OS-SS-20
OS-SS-21
OS-SS-22
OS-SS-23
OS-SS-24

$6.33 \pm 1.00$

$7.00 \pm 1.00$

$7.00 \pm 1.00$

$6.67 \pm 1.41$

$8.33 \pm 1.73$

$8.00 \pm 1.41$

$8.33 \pm 1.41$

$8.33 \pm 1.00$

$7.33 \pm 1.00$

$6.33 \pm 1.00$

$7.00 \pm 1.41$

$7.67 \pm 1.00$

$6.00 \pm 1.00$

$8.00 \pm 1.00$

$6.33 \pm 1.00$

$6.33 \pm 1.00$

$10.33 \pm 1.73$

$8.00 \pm 1.41$

$6.00 \pm 1.00$

$7.33 \pm 1.00$

$5.67 \pm 1.00$

$7.00 \pm 1.73$

$6.67 \pm 1.41$

$7.00 \pm 1.00$

$510.00 \pm 10.00$

$4.00 \pm 3.00$

$4.00 \pm 1.00$

$5.00 \pm 1.00$

$5.00 \pm 1.00$

$9.00 \pm 1.00$

$10.00 \pm 2.00$

$6.00 \pm 1.00$

$10.00 \pm 2.00$

$9.00 \pm 2.00$

$11.00 \pm 2.00$

$8.00 \div 1.00$

$8.00 \pm 1.00$

$8.00 \pm 1.00$

$7.00 \pm 1.00$

$11.00 \div 2.00$

$8.00 \pm 1.00$

$7.00 \div 1.00$

$5.00 \pm 1.00$

$9.00 \div 1.00$

$7.00 \div 1.00$

$4.00 \div 1.00$

$5.00 \pm 1.00$

$6.00 \pm 1.00$

$5.00 \div 1.00$

$5.00 \pm 1.00$

$7.00 \div 1.00$

$7.00 \pm 1.00$

$7.00 \pm 1.00$

$7.00 \pm 1.00$

$5.00 \pm 1.00$

$6.00 \pm 1.00$

$4.00 \pm 1.00$

$7.00 \pm 1.00$

$5.00 \pm 1.00$

$6.00 \pm 1.00$

$5.00 \pm 1.00$

$5.00 \pm 1.00$

$5.00 \pm 1.00$

$9.00 \pm 2.00$

$7.00 \pm 1.00$

$5.00 \pm 1.00$

$5.00 \pm 1.00$

$4.00 \pm 1.00$

$5.00 \pm 1.00$

$6.00 \pm 1.00$

$6.00 \pm 1.00$ 
Table XX1. Summary of Uranium-234 in Soil for 1988 (Cont 'd)

\begin{tabular}{|c|c|c|c|c|}
\hline $\begin{array}{l}\text { Sample } \\
\text { Location }\end{array}$ & $\begin{array}{r}\text { No. of } \\
\text { Samples }\end{array}$ & $\begin{array}{c}\text { Average uCi/g } \\
\left(10^{-7}\right)\end{array}$ & $\begin{array}{c}\text { Maximum uCi/g } \\
\left(10^{-7}\right)\end{array}$ & $\begin{array}{c}\text { Minimum uCi/g } \\
\left(10^{-7}\right)\end{array}$ \\
\hline \multicolumn{5}{|c|}{ Off Site (Cont'd) } \\
\hline $\begin{array}{l}\text { OS-SS-25 } \\
\text { OS-SS-26 } \\
\text { OS-SS-27 } \\
\text { OS-SS-28 } \\
\text { OS-SS-29 } \\
\text { OS-SS-30 } \\
\text { OS-SS-31 }\end{array}$ & $\begin{array}{l}3 \\
3 \\
3 \\
3 \\
3 \\
3 \\
3\end{array}$ & $\begin{array}{l}6.67 \pm 1.00 \\
6.67 \pm 1.00 \\
6.67 \pm 1.00 \\
6.00 \pm 1.00 \\
7.33 \pm 1.00 \\
8.00 \pm 1.00 \\
7.33 \pm 1.41\end{array}$ & $\begin{array}{r}7.00 \pm 1.00 \\
8.00 \pm 1.00 \\
8.00 \pm 1.00 \\
7.00 \pm 1.00 \\
8.00 \pm 1.00 \\
10.00 \pm 1.00 \\
9.00 \pm 2.00\end{array}$ & $\begin{array}{l}6.00 \pm 1.00 \\
6.00 \pm 1.00 \\
6.00 \pm 1.00 \\
5.00 \div 1.00 \\
6.00 \pm 1.00 \\
6.00 \pm 1.00 \\
6.00 \pm 1.00\end{array}$ \\
\hline \multicolumn{5}{|c|}{ Control } \\
\hline OS-SS-32 & 13 & $7.00 \pm 1.66$ & $9.00 \pm 1.00$ & $5.00 \div 1.00$ \\
\hline
\end{tabular}

Minimum Detectable Limit $=2 \times 10^{-8} \mathrm{uCi} / \mathrm{g}$

Reported errors are counting errors at the $95 \%$ confidence level

Activity is per gram of dry soll

Sample locations are sited on Figures 7, 8, 9, and 10. Sample site BU-SS-01 was located at the

Bushland Agriculture Research Station (Figure 1) 
Table XXI1. Summary of Uranium-238 in Soil for 1988

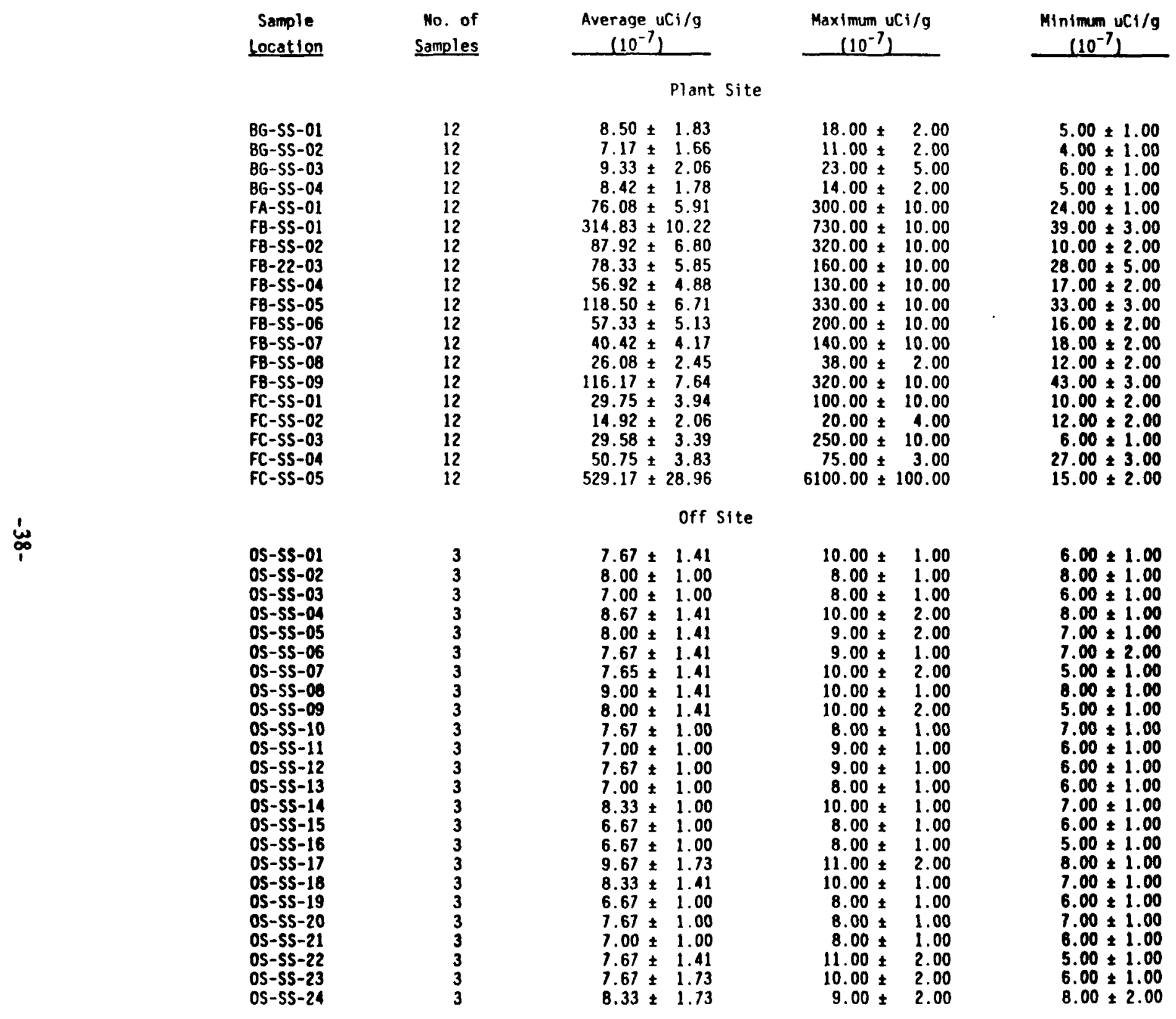


Table XX11. Summary of Uranium-238 in Soil for 1988 (Cont'd)

\begin{tabular}{|c|c|c|c|c|c|}
\hline $\begin{array}{c}\text { Sample } \\
\text { Location }\end{array}$ & $\begin{array}{r}\text { No. of } \\
\text { Samples }\end{array}$ & $\begin{array}{c}\text { Average } u C i / g \\
\left(10^{-7}\right)\end{array}$ & \multicolumn{2}{|c|}{$\begin{array}{c}\text { Maximum } u C i / g \\
\left(10^{-7}\right) \\
\end{array}$} & \multirow[t]{2}{*}{$\begin{array}{c}\text { Minimum } u C l / g \\
\left(10^{-7}\right)\end{array}$} \\
\hline \multicolumn{5}{|c|}{ Off Site (Cont $\left.{ }^{\circ} d\right)$} & \\
\hline $\begin{array}{l}O S-S S-25 \\
0 S-5 S-26 \\
0 S-S S-27 \\
O S-5 S-28 \\
O S-5 S-29 \\
O S-S S-30 \\
O S-S S-31\end{array}$ & $\begin{array}{l}3 \\
3 \\
3 \\
3 \\
3 \\
3 \\
3\end{array}$ & $\begin{array}{l}7.00 \pm 1.00 \\
6.00 \pm 1.00 \\
7.00 \pm 1.00 \\
6.33 \pm 1.00 \\
8.33 \pm 1.00 \\
8.33 \pm 1.41 \\
7.33 \pm 1.73\end{array}$ & $\begin{array}{r}8.00 \pm \\
9.00 \pm \\
8.00 \pm \\
7.00 \pm \\
10.00 \pm \\
10.00 \pm \\
9.00 \pm\end{array}$ & $\begin{array}{l}1.00 \\
1.00 \\
1.00 \\
1.00 \\
1.00 \\
1.00 \\
2.00\end{array}$ & $\begin{array}{l}5.00 \pm 1.00 \\
4.00 \pm 1.00 \\
6.00 \pm 1.00 \\
6.00 \pm 1.00 \\
7.00 \pm 1.00 \\
6.00 \pm 1.00 \\
5.00 \pm 1.00\end{array}$ \\
\hline \multicolumn{6}{|c|}{ Control } \\
\hline OS-SS-32 & 13 & $6.92 \pm 1.73$ & $9.00 \pm$ & 1.00 & $5.00 \pm 1.00$ \\
\hline
\end{tabular}

Minimum Detectable Limit $=2 \times 10^{-8} \mathrm{uCi} / \mathrm{g}$

Reported errors are counting errors at the $95 \%$ confidence level

Activity is per gram of dry soll

Sample locations are sited on Figures 7, 8, 9, and 10. Sample site BU-SS-01 was located at the

Bushland Agriculture Research Station (Figure 1) 
Table XXI11. Summary of Tritium in Vegetation for 1988

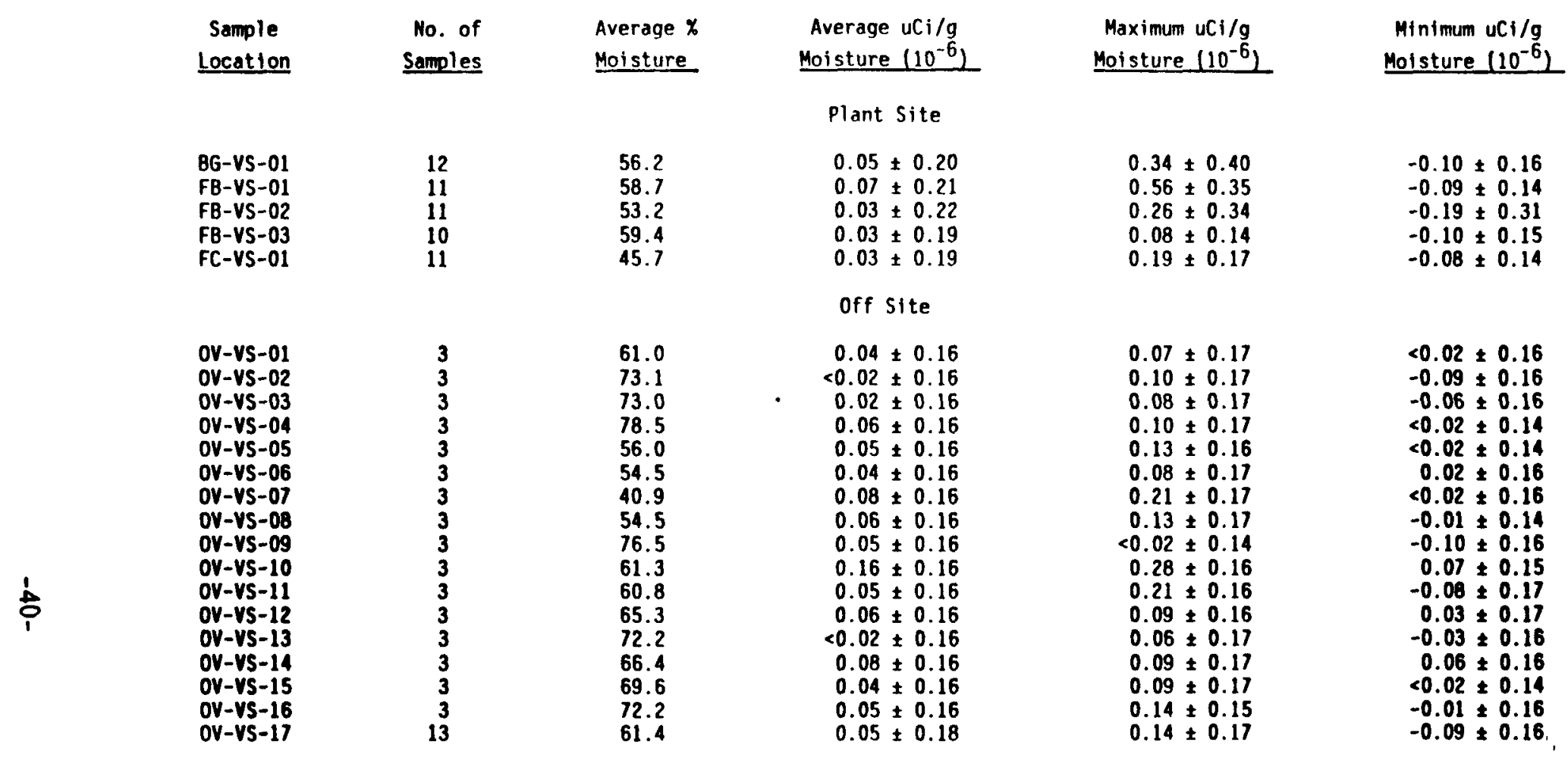

Minimen Datectable Limit $=0.02 \times 10^{-6} \mathrm{uCi} / \mathrm{g}$ moisture

Reported errors are counting errors at the $95 \%$ confidence level

Sample locations are sited on Figures 7, 8, 9, and 10. Sample site BU-VS-01 was located at the Sample located at the

Bushland Agrlculture Research Station (Figure 1) 
Table XXIV. Summary of Uranium-234 in Vegetation for 1988

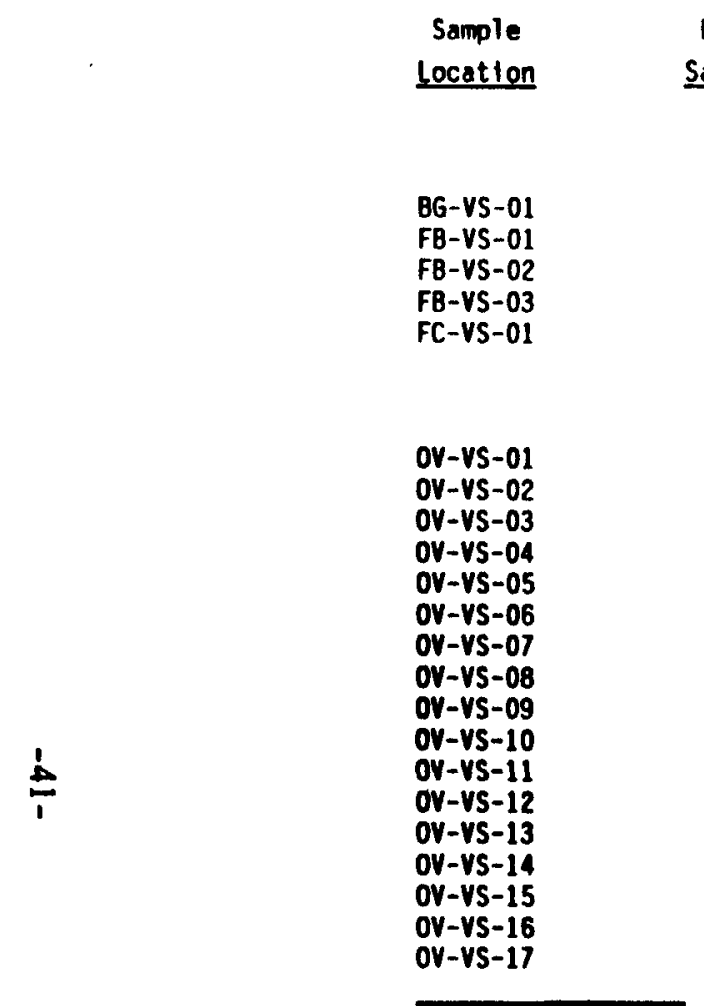

No. of

Average $\mathrm{uCi} / \mathrm{g}$

Maximum $u C i / g$

Samples

$\left(10^{-7}\right)$

$\left(10^{-7}\right.$

Minimum uCi/g

Plant Site

$\begin{array}{ll}12 & 1.06 \pm 0.30 \\ 11 & 1.90 \pm 0.30 \\ 11 & 4.19 \pm 0.54 \\ 10 & 5.05 \pm 0.62 \\ 11 & 0.96 \pm 0.26\end{array}$

$2.70 \pm 0.50$
$6.30 \pm 0.50$
$11.00 \pm 1.00$
$12.00 \pm 1.00$

$0.20 \pm 0.10$

$0.30 \pm 0.10$

$0.30 \pm 0.10$

off Site

OV-VS-0

OV-VS -03

$3.20 \pm 0.50$

$0.10 \pm 0.10$

Minimim Datectable Limit $=4 \times 10^{-8} \mathrm{uCt} / \mathrm{g}$

Reported arrors are counting errors at the $95 \%$ confidence level

Results are per gram dry vegetation

$2.90 \pm 0.68 \quad 3.20 \pm 0.70$

$2.20 \pm 0.41$

$2.90 \pm 0.50$

$0.70 \pm 0.20$

$38.00 \pm 2.00$

$13.67 \pm 1.19$

$1.97 \pm 0.42$

$3.60 \pm 0.60$

$0.63 \pm 0.14$

$3.60 \pm 0.60$

$1.00 \pm 0.20$

$3.10 \pm 0.60$
$2.90 \pm 1.00$

$2.03 \pm 0.45$

$3.97 \pm 0.96$

$3.07 \pm 0.62$

$2.90 \pm 1.00$

$6.00 \pm 1.50$
$5.00 \pm 0.80$

$3.30 \pm 0.70$

$1.70 \pm 0.41$

$1.83 \pm 0.48$

$2.10 \pm 0.39$

$2.40 \pm 0.50$

$2.30 \pm 0.60$

$3.10 \pm 0.50$

$2.60 \pm 0.50$

$.67 \pm 0.34$

$2.60 \pm 0.50$
$2.20 \pm 0.40$

$2.20 \pm 0.40$
$2.50 \pm 0.50$

$2.40 \pm 0.50$
$1.50 \pm 0.30$
$0.30 \pm 0.10$
$0.40 \pm 0.10$
$0.20 \pm 0.10$
$0.20 \pm 0.10$
$0.30 \pm 0.10$
$0.90 \pm 0.20$
$2.00 \pm 0.40$
$1.00 \pm 0.20$
$1.00 \pm 0.20$
$0.90 \pm 0.30$
$1.10 \pm 0.30$
$1.30 \pm 0.20$
$0.30 \pm 0.10$
$0.50 \pm 0.20$
$0.30 \pm 0.10$

Sample locations are sited on Figures 7, 8, 9, and 10. Sample site BU-VS-01 was located at the Bushland Agriculture Research Station (Figure 1) 
Table XXV. Summary of Uranium-238 in Vegetation for 1988

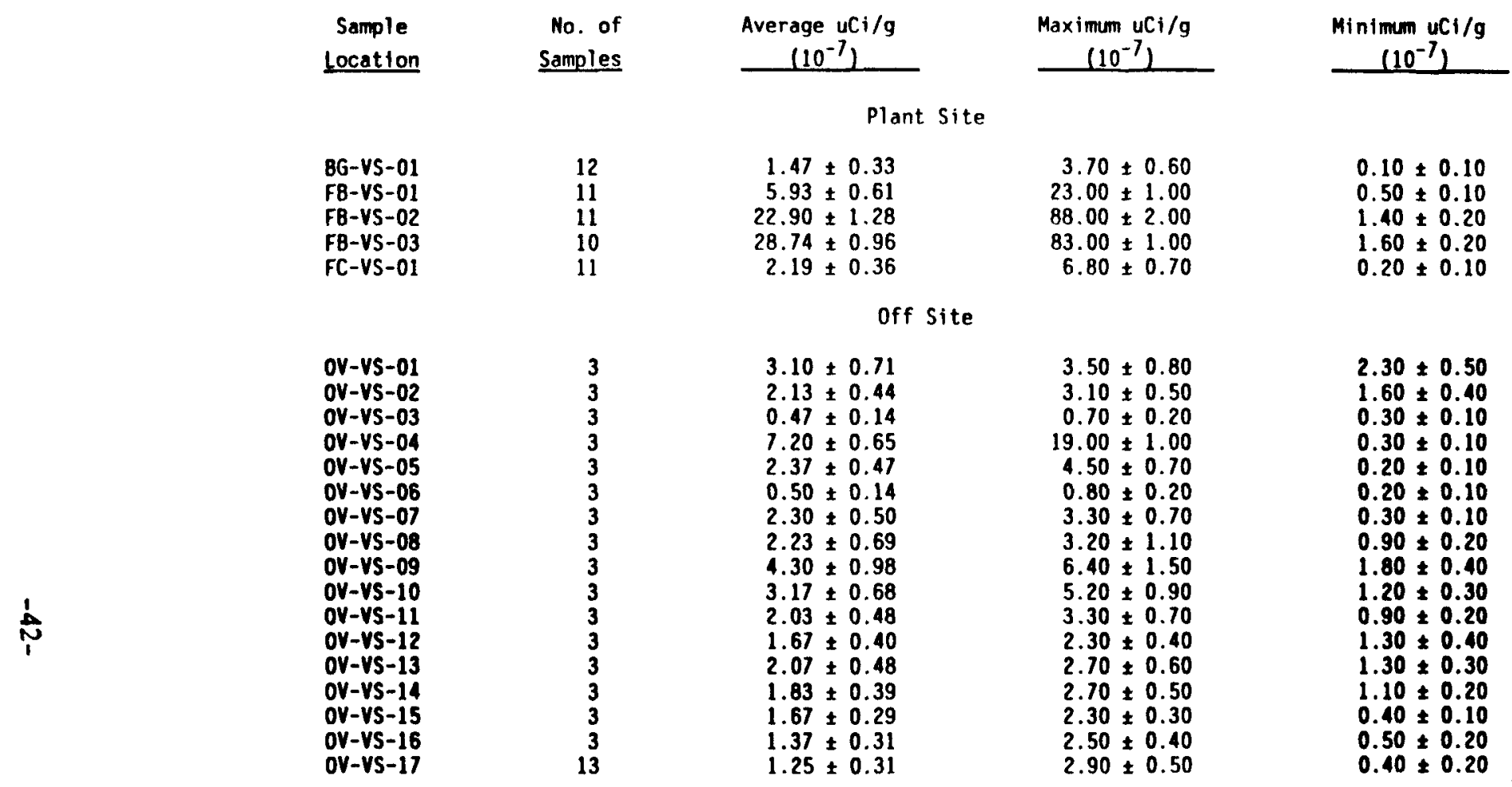

Minimim Detectable Limit $=4 \times 10^{-8} \mathrm{uCi} / \mathrm{g}$

Reported errors are counting errors at the $95 \%$ confidence level

Results are per gran dry vegetation

Sample locations are sited on Figures 7, 8, 9, and 10. Sample site BU-VS-01 was located at the Bushland Agriculture Research Station (Fłgure i) 
Table XXVI. Surmary of Fluoride in Vegetation

\begin{tabular}{|c|c|c|c|c|}
\hline \multirow{2}{*}{$\begin{array}{c}\text { Sample } \\
\text { Location }\end{array}$} & \multirow{2}{*}{$\begin{array}{r}\text { Number } \\
\text { Samples }\end{array}$} & \multicolumn{3}{|c|}{$\mathrm{mg} / \mathrm{l}$} \\
\hline & & Average & Maximum & Minimum \\
\hline $\begin{array}{l}\text { BG-VS-01 } \\
\text { OV-VS-02 } \\
\text { OV-VS-06 } \\
\text { OV-VS-10 } \\
\text { OV-VS-14 } \\
\text { OV-VS-17 }\end{array}$ & $\begin{array}{r}11 \\
2 \\
2 \\
2 \\
2 \\
12\end{array}$ & $\begin{array}{l}53.27 \\
76.00 \\
37.00 \\
75.00 \\
53.50 \\
60.08\end{array}$ & $\begin{array}{r}130.00 \\
110.00 \\
39.00 \\
105.00 \\
62.00 \\
170.00\end{array}$ & $\begin{array}{r}<.00 \\
42.00 \\
35.00 \\
45.00 \\
45.00 \\
4.00\end{array}$ \\
\hline
\end{tabular}

Minimum detectable $1 \mathrm{imit}=3.0 \mathrm{mg} / 1$.

Table XXVII. Sumnary of Tritium in Jackrabbits for 1988

\begin{tabular}{|c|c|c|c|c|}
\hline $\begin{array}{c}\text { Specimen } \\
\text { Identification }\end{array}$ & $\begin{array}{c}\text { Kidney } \\
\left(10^{-6} \mathrm{uct} / \mathrm{ml}\right)\end{array}$ & $\begin{array}{c}\text { Liver } \\
\left(10^{-6} \mathrm{uCj} / \mathrm{ml}\right)\end{array}$ & $\left(10^{-6} \cup \mathrm{Ci} / \mathrm{ml}\right)$ & $\begin{array}{l}\text { (Flesh)Muscle } \\
\left(10^{-6} \mathrm{yCl} / \mathrm{ml}\right)\end{array}$ \\
\hline Zone 12 (1) & $\begin{array}{l}<0.02 \pm 0.16 \\
<0.11 \pm 0.25\end{array}$ & $\begin{array}{r}0.12 \pm 0.16 \\
<0.03 \pm 0.15\end{array}$ & $\begin{array}{l}<0.04 \pm 0.15 \\
<0.05 \pm 0.15\end{array}$ & $\begin{array}{l}<0.09 \pm 0.16 \\
<0.12 \pm 0.16\end{array}$ \\
\hline Ftring site (1) & $\begin{array}{r}0.32 \pm 0.64 \\
<0.01 \pm 0.16\end{array}$ & $\begin{aligned}<0.09 & \pm 0.15 \\
0.12 & \pm 0.16\end{aligned}$ & $\begin{array}{l}<0.07 \pm 0.15 \\
<0.03 \pm 0.16\end{array}$ & $\begin{array}{l}0.04 \pm 0.16 \\
0.06 \pm 0.16\end{array}$ \\
\hline $\begin{array}{r}\text { Zone } 4 \text { (1) } \\
\text { (2) }\end{array}$ & $\begin{array}{r}0.00 \pm 0.16 \\
<0.02 \pm 0.16\end{array}$ & $\begin{array}{l}<0.10 \pm 0.15 \\
<0.03 \pm 0.15\end{array}$ & $\begin{array}{l}<0.07 \pm 0.15 \\
<0.06 \pm 0.15\end{array}$ & $\begin{array}{l}<0.02 \pm 0.16 \\
<0.06 \pm 0.16\end{array}$ \\
\hline $\begin{array}{ll}\text { Sumage Treatment } & \text { (1) } \\
\text { Plant } & \text { (2) }\end{array}$ & $\begin{aligned}<0.23 & \pm 0.42 \\
0.05 & \pm 0.16\end{aligned}$ & $\begin{array}{r}0.02 \pm 0.16 \\
<0.08 \pm 0.15\end{array}$ & $\begin{array}{l}0.07 \pm 0.15 \\
0.00 \pm 0.15\end{array}$ & $\begin{aligned}<0.02 & \pm 0.16 \\
0.02 & \pm 0.16\end{aligned}$ \\
\hline $\begin{array}{l}\text { Retention Basin (1) } \\
\text { Playa }\end{array}$ & $\begin{array}{l}0.02 \pm 0.16 \\
0.08 \pm 0.32\end{array}$ & $\begin{array}{l}<0.04 \pm 0.16 \\
<0.04 \pm 0.15\end{array}$ & $\begin{array}{l}<0.04 \pm 0.15 \\
<0.10 \pm 0.15\end{array}$ & $\begin{aligned}<0.09 & \pm 0.16 \\
0.02 & \pm 0.16\end{aligned}$ \\
\hline
\end{tabular}

Specimen Identification sites are shown in Figure 2.

No control Jackrabbit for 1988 .

Iritlum is extracted liquid with units $=m 1$. Refer to $x$ moisture Table $x \times x 11$. 
Table XXVIII. Summary of Plutonium-239 in Jackrabbit for 1988

\begin{tabular}{|c|c|c|c|c|c|c|}
\hline $\begin{array}{c}\text { Specimen } \\
\text { Identification }\end{array}$ & $\begin{array}{c}\text { Kidney } \\
\left(10^{-6} u C i / g\right)^{*}\end{array}$ & 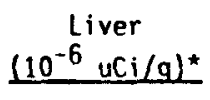 & $\begin{array}{c}\text { Lung } \\
\left(10^{-6} u C j / g\right)^{\star}\end{array}$ & $\begin{array}{l}\text { (Flesh)Muscle } \\
\left(10^{-6} \cup \mathrm{Ci} / \mathrm{g}\right)^{\star}\end{array}$ & $\begin{array}{r}80^{-6} \\
\end{array}$ & $\begin{array}{l}\text { one } \\
u C 1 /\left.q\right|^{*}\end{array}$ \\
\hline Zone $12(1)$ & $\begin{array}{l}0.2 \pm 0.2 \\
0.1 \pm 0.1\end{array}$ & $\begin{array}{l}0.0 \pm 0.1 \\
0.0 \pm 0.1\end{array}$ & $\begin{array}{l}0.0 \pm 0.1 \\
0.0 \pm 0.1\end{array}$ & $\begin{array}{l}0.01 \pm 0.1 \\
0.0 \pm 0.1\end{array}$ & $\begin{array}{l}0.0 \\
0.0\end{array}$ & $\begin{array}{l} \pm 0.1 \\
\pm 0.1\end{array}$ \\
\hline Firing site (1) & $\begin{array}{l}0.1 \pm 0.1 \\
0.0 \pm 0.1\end{array}$ & $\begin{array}{l}0.0 \pm 0.1 \\
0.0 \pm 0.1\end{array}$ & $\begin{array}{l}0.0 \pm 0.1 \\
0.2 \pm 0.2\end{array}$ & $\begin{array}{l}0.0 \pm 0.1 \\
0.0 \pm 0.1\end{array}$ & $\begin{array}{l}0.0 \\
0.0\end{array}$ & $\begin{array}{l} \pm 0.1 \\
\pm 0.1\end{array}$ \\
\hline Zone $4(1)$ & $\begin{array}{l}0.1 \pm 0.2 \\
0.1 \pm 0.1\end{array}$ & $\begin{array}{l}0.0 \pm 0.1 \\
0.0 \pm 0.1\end{array}$ & $\begin{array}{l}0.0 \pm 0.1 \\
0.0 \pm 0.1\end{array}$ & $\begin{array}{l}0.0 \pm 0.1 \\
0.0 \pm 0.1\end{array}$ & $\begin{array}{l}0.0 \\
0.0\end{array}$ & $\begin{array}{l} \pm 0.1 \\
\pm 0.1\end{array}$ \\
\hline $\begin{array}{cc}\text { Sewage Treatment } & (1) \\
\text { Plant } & (2)\end{array}$ & $\begin{array}{l}0.0 \pm 0.1 \\
0.3 \pm 0.2\end{array}$ & $\begin{array}{l}0.0 \pm 0.1 \\
0.0 \pm 0.1\end{array}$ & $\begin{array}{l}0.0 \pm 0.1 \\
0.0 \pm 0.1\end{array}$ & $\begin{array}{l}0.0 \pm 0.1 \\
0.0 \pm 0.1\end{array}$ & $\begin{array}{l}0.0 \\
0.0\end{array}$ & $\begin{array}{l} \pm 0.1 \\
\pm 0.1\end{array}$ \\
\hline $\begin{array}{l}\text { Retention Basin (1) } \\
\text { Playa }\end{array}$ & $\begin{array}{lll}0.1 & \pm 0.1 \\
0.0 & \pm 0.1\end{array}$ & $\begin{array}{l}0.0 \pm 0.1 \\
0.0 \pm 0.1\end{array}$ & $\begin{array}{lll}0.0 & \pm 0.1 \\
0.0 & \pm 0.1\end{array}$ & $\begin{array}{l}0.0 \pm 0.1 \\
0.0 \pm 0.1\end{array}$ & $\begin{array}{l}0.0 \\
0.0\end{array}$ & $\begin{array}{l} \pm 0.1 \\
\pm 0.1\end{array}$ \\
\hline
\end{tabular}

tAsh

Specimen identification sites are shown in Figure 2.

No control Jackrabbit for 1988.

Table XXIX. Summary of Uranium-234 in Jackrabbit for 1988

\begin{tabular}{|c|c|c|c|c|c|}
\hline $\begin{array}{c}\text { Specimen } \\
\text { Identification }\end{array}$ & $\begin{array}{c}\text { Kidney } \\
\left(10^{-6} \text { uci/g)* }\right.\end{array}$ & $\begin{array}{c}\text { Liver } \\
\left(10^{-6} \text { uci/g)* }\right.\end{array}$ & $\begin{array}{c}\text { Lung } \\
\left(10^{-6} \mathrm{uCl} / \mathrm{a}\right)^{*}\end{array}$ & $\begin{array}{l}\text { (Flesh)Muscle } \\
\left(10^{-6} \text { uci/a) }\right.\end{array}$ & $\begin{array}{c}\text { Bone } \\
\left(10^{-6}+(1 / 0)\right.\end{array}$ \\
\hline Zone 12 (1) & $\begin{aligned}<0.3 & \pm 0.8 \\
0.6 & \pm 0.9\end{aligned}$ & $\begin{array}{l}0.0 \pm 0.1 \\
0.0 \pm 0.2\end{array}$ & $\begin{array}{l}0.0 \pm 0.1 \\
0.0 \pm 0.2\end{array}$ & $\begin{array}{l}0.1 \pm 0.1 \\
0.0 \pm 0.1\end{array}$ & $\begin{array}{l}0.0 \pm 0.1 \\
0.0 \pm 0.1\end{array}$ \\
\hline Firing site $\left(\begin{array}{l}1 \\
(2)\end{array}\right)$ & $\begin{array}{l}0.1 \pm 0.4 \\
0.3 \pm 0.9\end{array}$ & $\begin{aligned} 0.0 & \pm 0.1 \\
<0.1 & \pm 0.8\end{aligned}$ & $\begin{array}{l}0.0 \pm 0.1 \\
0.2 \pm 0.8\end{array}$ & $\begin{array}{l}0.2 \pm 0.1 \\
0.1 \pm 0.1\end{array}$ & $\begin{array}{l}0.3 \pm 0.1 \\
0.0 \pm 0.1\end{array}$ \\
\hline Zone 4 (1) & $\begin{array}{l}0.0 \pm 0.4 \\
0.4 \pm 0.5\end{array}$ & $\begin{array}{l}0.2 \pm 0.1 \\
0.0 \pm 0.1\end{array}$ & $\begin{array}{l}0.3 \pm 0.3 \\
0.0 \pm 0.2\end{array}$ & $\begin{array}{l}0.0 \pm 0.1 \\
0.1 \pm 0.1\end{array}$ & $\begin{array}{l}0.3 \pm 0.1 \\
0.3 \pm 0.1\end{array}$ \\
\hline $\begin{array}{l}\text { Semage Ireatment (1) } \\
\text { Plant }\end{array}$ & $\begin{array}{l}<0.2 \pm 0.4 \\
<0.2 \pm 0.4\end{array}$ & $\begin{array}{l}0.0 \pm 0.4 \\
0.0 \pm 0.1\end{array}$ & $\begin{array}{l}0.6 \pm 0.9 \\
0.1 \pm 0.2\end{array}$ & $\begin{array}{l}0.1 \pm 0.1 \\
0.2 \pm 0.1\end{array}$ & $\begin{array}{l}0.0 \pm 0.1 \\
0.0 \pm 0.1\end{array}$ \\
\hline $\begin{array}{l}\text { Rotention Basin (1) } \\
\text { Playa }\end{array}$ & $\begin{array}{l}0.0 \pm 0.3 \\
0.0 \pm 0.8\end{array}$ & $\begin{array}{l}0.0 \pm 0.1 \\
0.0 \pm 0.2\end{array}$ & $\begin{aligned}<0.1 & \pm 0.2 \\
0.1 & \pm 0.4\end{aligned}$ & $\begin{array}{l}0.1 \pm 0.1 \\
0.3 \pm 0.1\end{array}$ & $\begin{array}{l}0.0 \pm 0.1 \\
0.0 \pm 0.1\end{array}$ \\
\hline
\end{tabular}

*Ash

Specimen Identiflcation sites are shown in Figure 2.

No control Jackrabblt for 1988. 
Table XXX. Sumary of Uranium-238 in Jackrabbit for 1988

\begin{tabular}{|c|c|c|c|c|c|c|c|c|}
\hline $\begin{array}{c}\text { Specimen } \\
\text { Identification }\end{array}$ & $\begin{array}{c}\text { Kidney } \\
\left(10^{-6} \mathrm{uCi} / \mathrm{a}\right)^{*}\end{array}$ & $\begin{array}{r}\mathrm{L} \\
40^{-6}\end{array}$ & $\begin{array}{l}\text { iver } \\
u(i / q)^{*}\end{array}$ & $\begin{array}{c}\text { Lung } \\
\left(10^{-6} \mathrm{uCl} / \mathrm{g}\right)^{*}\end{array}$ & $\begin{array}{l}\text { (Fles } \\
110^{-6} \\
\end{array}$ & $\begin{array}{l}\text { h)Muscle } \\
\text { uct/al* }\end{array}$ & $\begin{array}{r}\quad 8 \\
-6 \\
\end{array}$ & $\begin{array}{l}\text { one } \\
u(1 / a)^{*}\end{array}$ \\
\hline Zone 12 (1) & $\begin{array}{l}<0.3 \pm 0.6 \\
<0.2 \pm 0.7\end{array}$ & $\begin{array}{l}0.0 \\
0.1\end{array}$ & $\begin{array}{l} \pm 0.1 \\
\pm 0.2\end{array}$ & $\begin{array}{l}0.0 \pm 0.1 \\
0.0 \pm 0.2\end{array}$ & $\begin{array}{l}0.0 \\
0.0\end{array}$ & $\begin{array}{l} \pm 0.1 \\
\pm 0.1\end{array}$ & $\begin{array}{l}0.0 \\
0.0\end{array}$ & $\begin{array}{l} \pm 0.1 \\
\pm 0.1\end{array}$ \\
\hline Firing site (1) & $\begin{array}{l}0.0 \pm 0.3 \\
0.1 \pm 0.8\end{array}$ & $\begin{array}{l}0.1 \\
0.1\end{array}$ & $\begin{array}{l} \pm 0.1 \\
\pm 0.7\end{array}$ & $\begin{aligned} & 0.0 \pm 0.1 \\
&<0.2 \pm 0.7\end{aligned}$ & $\begin{array}{l}0.1 \\
0.0\end{array}$ & $\begin{array}{l} \pm 0.1 \\
\pm 0.1\end{array}$ & $\begin{array}{l}0.0 \\
0.0\end{array}$ & $\begin{array}{l} \pm 0.1 \\
\pm 0.1\end{array}$ \\
\hline $\begin{array}{r}\text { Zone } 4(1) \\
(2)\end{array}$ & $\begin{array}{l}0.0 \pm 0.3 \\
0.3 \pm 0.4\end{array}$ & $\begin{array}{l}0.0 \\
0.0\end{array}$ & $\begin{array}{ll} \pm & 0.1 \\
\pm & 0.1\end{array}$ & $\begin{array}{l}0.3 \pm 0.2 \\
0.0 \pm 0.2\end{array}$ & $\begin{array}{l}0.0 \\
0.1\end{array}$ & $\begin{array}{l} \pm 0.1 \\
\pm 0.1\end{array}$ & $\begin{array}{l}0.0 \\
0.0\end{array}$ & $\begin{array}{l} \pm 0.1 \\
\pm 0.1\end{array}$ \\
\hline $\begin{array}{l}\text { Sewage Treatment (1) } \\
\text { Plant }\end{array}$ & $\begin{aligned}<0.1 & \pm 0.3 \\
0.0 & \pm 0.4\end{aligned}$ & $\begin{array}{l}0.1 \\
0.0\end{array}$ & $\begin{array}{ll} \pm & 0.4 \\
\pm & 0.1\end{array}$ & $\begin{array}{l}1.2 \pm 0.9 \\
0.0 \pm 0.2\end{array}$ & $\begin{array}{l}0.1 \\
0.0\end{array}$ & $\begin{array}{ll} \pm & 0.1 \\
\pm & 0.1\end{array}$ & $\begin{array}{l}0.0 \\
0.0\end{array}$ & $\begin{array}{l} \pm 0.1 \\
\pm 0.1\end{array}$ \\
\hline $\begin{array}{l}\text { Retention Basin (1) } \\
\text { Playa }\end{array}$ & $\begin{array}{l}<0.1 \pm 0.2 \\
<0.3 \pm 0.6\end{array}$ & $\begin{array}{l}0.0 \\
0.0\end{array}$ & $\begin{array}{l} \pm 0.1 \\
\pm 0.2\end{array}$ & $\begin{aligned} 0.0 & \pm 0.2 \\
<0.1 & \pm 0.3\end{aligned}$ & $\begin{array}{l}0.1 \\
0.1\end{array}$ & $\begin{array}{ll} \pm & 0.1 \\
\pm & 0.1\end{array}$ & $\begin{array}{l}0.0 \\
0.0\end{array}$ & $\begin{array}{l} \pm 0.1 \\
\pm 0.1\end{array}$ \\
\hline
\end{tabular}

\section{"Ash}

Speciment Identification sites are shown in Figure 2.

No control Jackrabbit for 1988 .

Table XXXI. Summary of Organ Weights of Jackrabbits for 1988

\begin{tabular}{|c|c|c|}
\hline $\begin{array}{c}\text { Specimen } \\
\text { Identification }\end{array}$ & $\begin{array}{l}\text { Kidney } \\
\text { (a) }\end{array}$ & $\begin{array}{l}\text { Liver } \\
\text { (a) }\end{array}$ \\
\hline Zone 12 (1) & $\begin{array}{r}17.5 \\
9.7\end{array}$ & $\begin{array}{l}68.7 \\
38.3\end{array}$ \\
\hline Firing site (1) & $\begin{array}{r}19.8 \\
4.8\end{array}$ & $\begin{array}{l}88.4 \\
17.3\end{array}$ \\
\hline Zone 4 (1) & $\begin{array}{l}19.7 \\
14.3\end{array}$ & $\begin{array}{r}109.9 \\
49.8\end{array}$ \\
\hline $\begin{array}{ll}\text { Sewage Treatment } & \text { (1) } \\
\text { Plant } & \text { (2) }\end{array}$ & $\begin{array}{r}6.6 \\
14.2\end{array}$ & $\begin{array}{l}27.8 \\
52.2\end{array}$ \\
\hline $\begin{array}{l}\text { Retention Basin (1) } \\
\text { Playa }\end{array}$ & $\begin{array}{l}17.2 \\
10.8\end{array}$ & $\begin{array}{l}90.6 \\
34.2\end{array}$ \\
\hline
\end{tabular}

Specimen identification sites are shown in Figure 2.

No control jackrabbit for 1988. 
Table XXXII. Summary of $\%$ Moisture of Jackrabbit Organs for 1988

\begin{tabular}{|c|c|c|}
\hline $\begin{array}{c}\text { Specimen } \\
\text { Identification }\end{array}$ & $\begin{array}{l}\text { Kidney } \\
(\%) \\
\end{array}$ & $\begin{array}{c}\text { (Flesh) Muscle } \\
(\%)\end{array}$ \\
\hline $\begin{array}{r}\text { Zone } 12(1) \\
(2)\end{array}$ & $\begin{array}{l}74.3 \\
72.2\end{array}$ & $\begin{array}{l}73.0 \\
63.6\end{array}$ \\
\hline Firing site (1) & 83.3 & $\begin{array}{l}65.5 \\
67.1\end{array}$ \\
\hline Zone 4 (1) & $\begin{array}{l}76.1 \\
83.9\end{array}$ & $\begin{array}{l}60.1 \\
75.4\end{array}$ \\
\hline $\begin{array}{ll}\text { Sewage Treatment } & (1) \\
\text { Plant } & (2)\end{array}$ & $\begin{array}{l}75.8 \\
77.5\end{array}$ & $\begin{array}{l}68.0 \\
77.2\end{array}$ \\
\hline $\begin{array}{c}\text { Retention Basin (1) } \\
\text { Playa }\end{array}$ & $\begin{array}{l}75.6 \\
83.3\end{array}$ & $\begin{array}{l}69.5 \\
69.5\end{array}$ \\
\hline
\end{tabular}

** Sample lost in lab accident.

Speciment Identification sites are shown in Figure 2.

No control jackrabbit for 1988 .

Table XXXIII. Sumary of $\mathcal{X}$ Ash of Jackrabbit Organs for 1988

\begin{tabular}{|c|c|c|c|c|}
\hline $\begin{array}{c}\text { Speciment } \\
\text { Identification }\end{array}$ & $\begin{array}{l}\text { Kidney } \\
(x)\end{array}$ & $\begin{array}{l}\text { Liver } \\
\underline{(x)}\end{array}$ & $\begin{array}{l}\text { Lung } \\
(x)\end{array}$ & $\begin{array}{l}\text { (Flesh) Muscle } \\
(x)\end{array}$ \\
\hline Zone 12 (1) & $\begin{array}{l}3.23 \\
5.56\end{array}$ & $\begin{array}{l}4.19 \\
4.44\end{array}$ & $\begin{array}{l}5.2 \\
8.7\end{array}$ & $\begin{array}{l}1.72 \\
1.70\end{array}$ \\
\hline Firing site (1) & $\begin{array}{r}1.88 \\
10.00\end{array}$ & $\begin{array}{l}4.59 \\
2.78\end{array}$ & $\begin{array}{r}6.2 \\
16.7\end{array}$ & $\begin{array}{l}1.40 \\
4.26\end{array}$ \\
\hline $\begin{array}{r}\text { Zone } 4(1) \\
(2)\end{array}$ & $\begin{array}{l}5.88 \\
7.41\end{array}$ & $\begin{array}{l}5.53 \\
6.25\end{array}$ & $\begin{array}{l}6.1 \\
7.3\end{array}$ & $\begin{array}{l}2.23 \\
1.51\end{array}$ \\
\hline $\begin{array}{ll}\text { Sewage Treatment (1) } \\
\text { Plant } & \text { (2) }\end{array}$ & $\begin{array}{r}18.18 \\
7.41\end{array}$ & $\begin{array}{l}3.13 \\
6.25\end{array}$ & $\begin{array}{l}5.3 \\
7.3\end{array}$ & $\begin{array}{l}1.78 \\
1.51\end{array}$ \\
\hline $\begin{array}{l}\text { Retention Basin (1) } \\
\text { Playa }\end{array}$ & $\begin{array}{l}8.82 \\
5.26\end{array}$ & $\begin{array}{l}4.93 \\
4.65\end{array}$ & $\begin{array}{l}3.9 \\
5.7\end{array}$ & $\begin{array}{l}1.72 \\
4.79\end{array}$ \\
\hline
\end{tabular}

Specimen identification sites are shown in figure 2. 
Table XXXIV. Summary of Chemical Analysis for Water Pollutants for 1988

\begin{tabular}{|c|c|c|c|c|c|c|c|}
\hline \multirow[b]{2}{*}{ Analysis } & Sample & No. of & \multicolumn{3}{|c|}{$(\mathrm{mg} / 1)$} & \multirow{2}{*}{$\begin{array}{l}\text { Minimum } \\
\text { Criteria } \\
\text { (ma/l) }\end{array}$} & \multirow{2}{*}{$\begin{array}{c}\text { Detectable } \\
\text { Limits } \\
(\mathrm{mg} / 1) \\
\end{array}$} \\
\hline & Identification & Samples & Average & Maximum & Minimum & & \\
\hline Acetone & & & & & & & 1.0 \\
\hline & $\begin{array}{l}\text { Playas } \\
\text { OW-WR-02 } \\
\text { OW-WR-06 } \\
\text { OW-UR-16 } \\
\text { OW-WR-18 }\end{array}$ & $\begin{array}{l}1 \\
1 \\
1 \\
1\end{array}$ & $\begin{array}{l}<1.0 \\
<1.0 \\
<1.0 \\
<1.0\end{array}$ & $\begin{array}{l}<1.0 \\
<1.0 \\
<1.0 \\
<1.0\end{array}$ & $\begin{array}{l}<1.0 \\
<1.0 \\
<1.0 \\
<1.0\end{array}$ & -.. & \\
\hline & $\begin{array}{l}\text { Plant Drinking Water } \\
\text { OW-WR-43 }\end{array}$ & 1 & $<1.0$ & $<1.0$ & $<1.0$ & --. & \\
\hline & $\begin{array}{l}\text { Monitor Wells } \\
\text { OW-WR-19 } \\
\text { OW-WR-20 } \\
\text { OW-WR-38 } \\
\text { OW-WR-39 } \\
\text { OW-WR-40 } \\
\text { OW-WR-44 }\end{array}$ & $\begin{array}{l}1 \\
1 \\
1 \\
1 \\
1 \\
1\end{array}$ & $\begin{array}{l}<1.0 \\
<1.0 \\
<1.0 \\
<1.0 \\
<1.0 \\
<1.0\end{array}$ & $\begin{array}{l}<1.0 \\
<1.0 \\
<1.0 \\
<1.0 \\
<1.0 \\
<1.0\end{array}$ & $\begin{array}{l}<1.0 \\
<1.0 \\
<1.0 \\
<1.0 \\
<1.0 \\
<1.0\end{array}$ & --- & \\
\hline & $\begin{array}{l}\text { Control } \\
\text { OW-WR-37 }\end{array}$ & 1 & $<1.0$ & $<1.0$ & $<1.0$ & --- & \\
\hline
\end{tabular}


Table XXXIV. Summary of Chemical Analysis for Water Pollutants for 1988 (Cont'd)

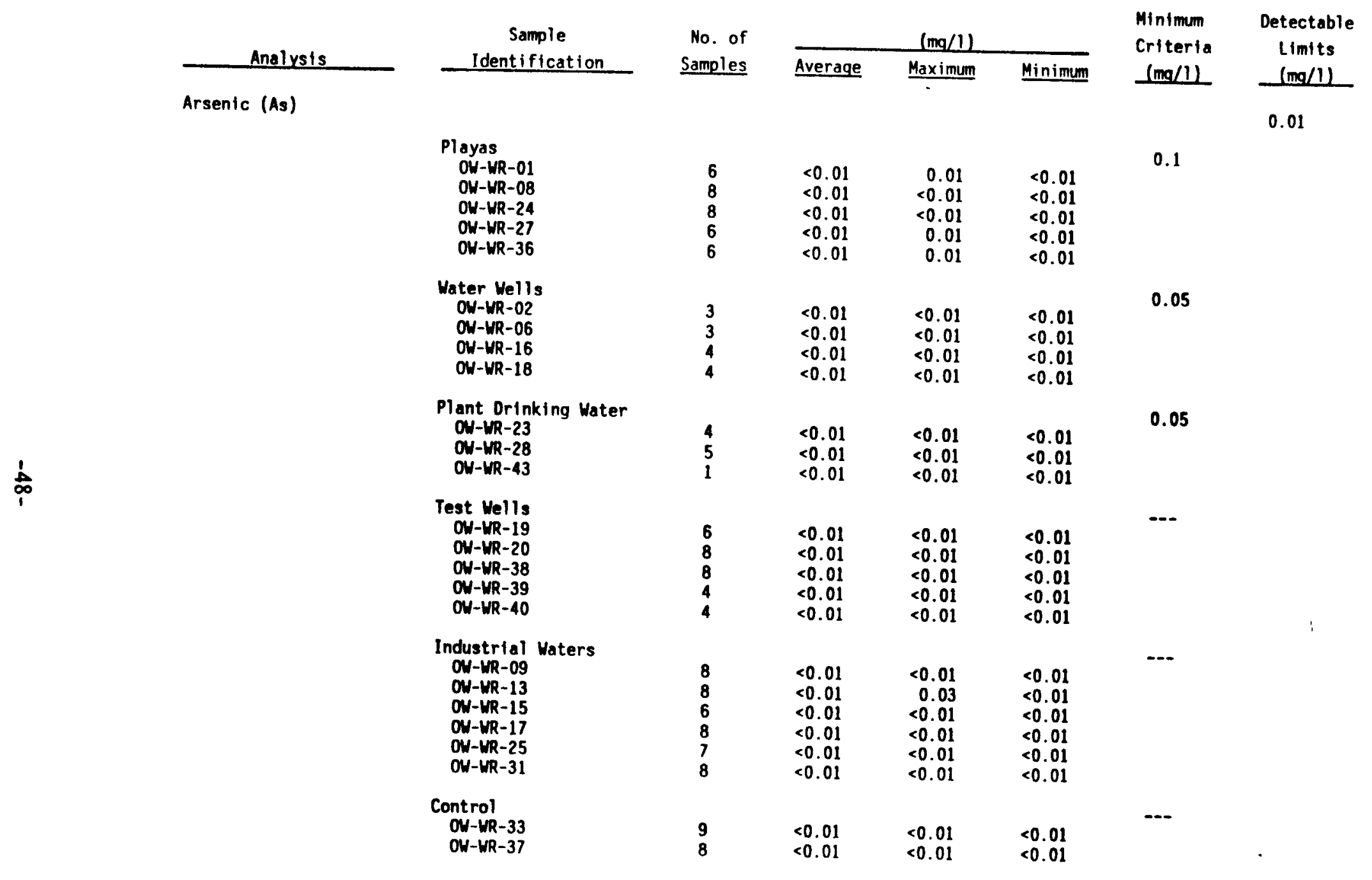


Table XXXIV. Summary of Chemical Analysis for Water Pollutants for 1988 (Cont'd)

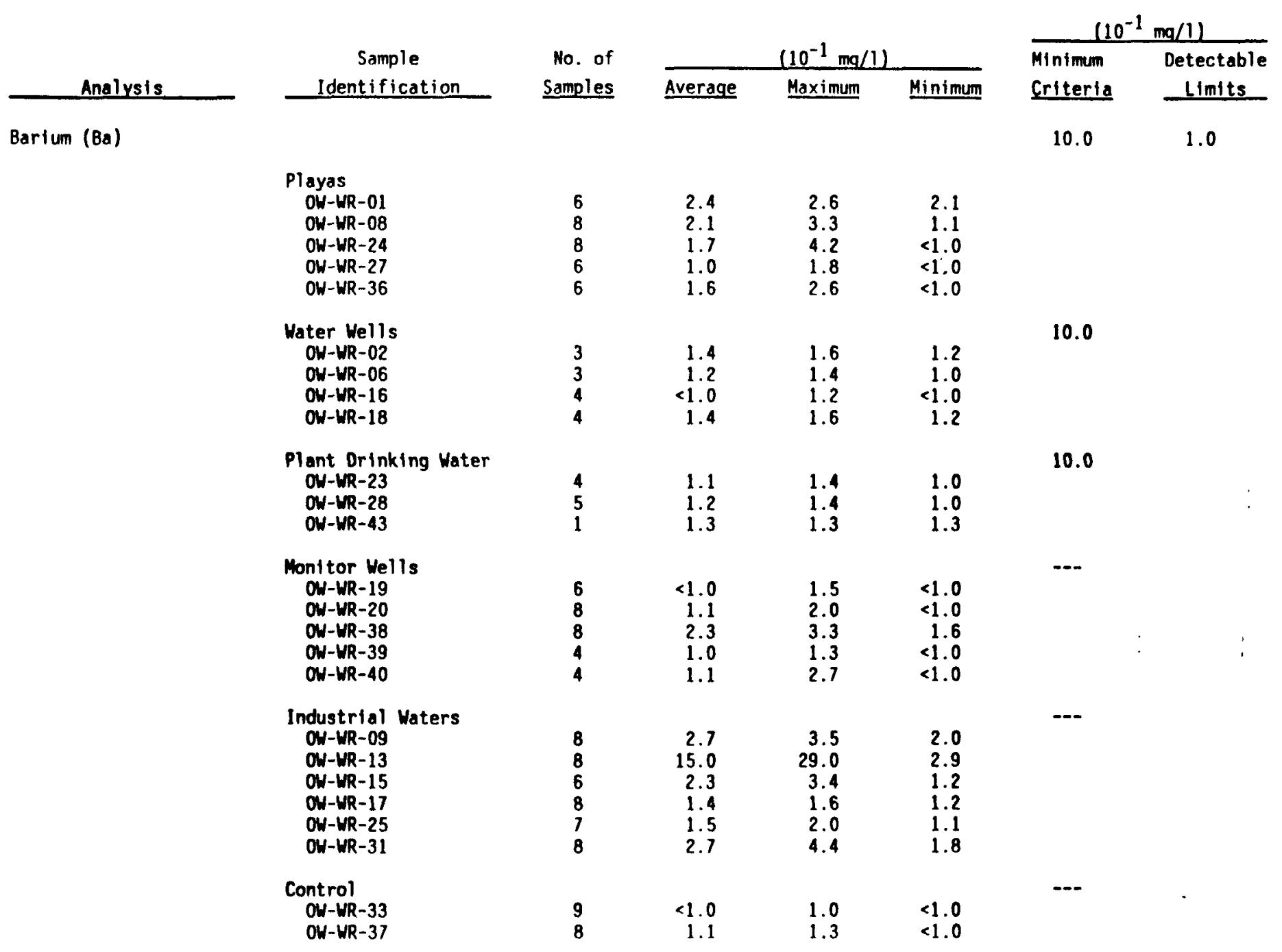


Table XXXIV. Summary of Chemical Analysis for Water Pollutants for 1988 (Cont'd)

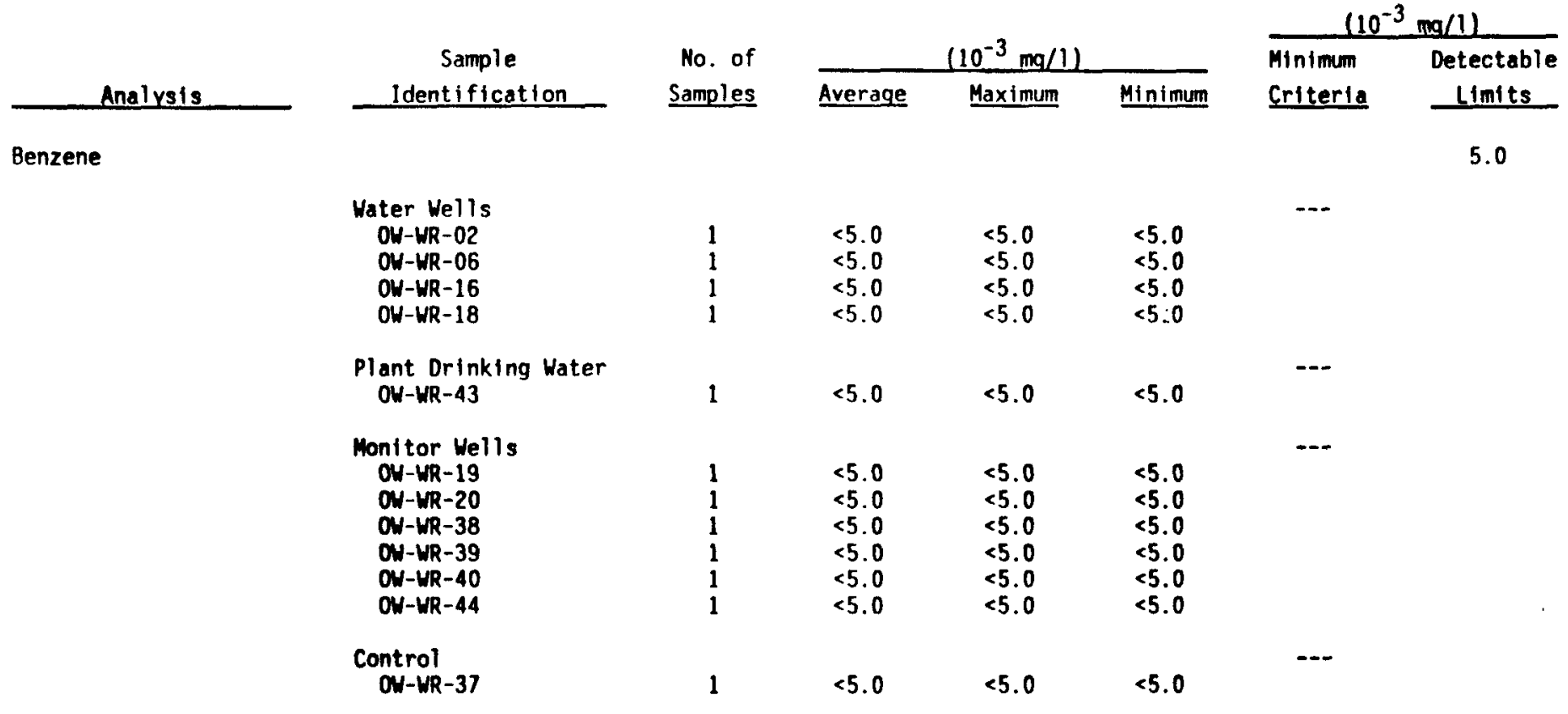


Table XXXIV. Sumary of Chemical Analysis for Water Pollutants for 1988 (Cont'd)

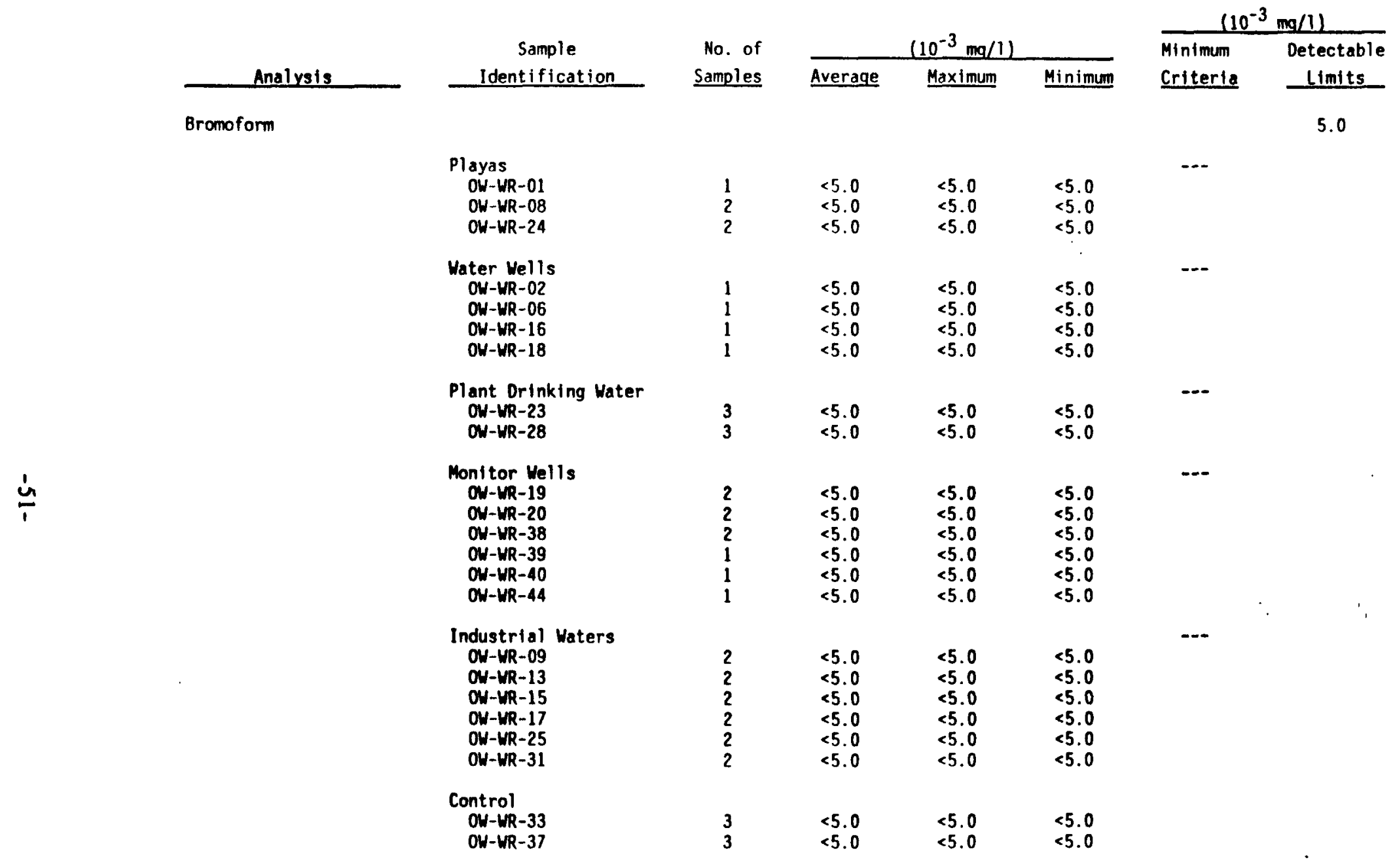


Table XXXIV. Sumary of Chemical Analysis for Water Pollutants for 1988 (Cont'd)

\begin{tabular}{|c|c|c|c|c|c|c|c|}
\hline \multirow[b]{3}{*}{ Analysis } & \multirow{3}{*}{$\begin{array}{c}\text { Sample } \\
\text { Identification }\end{array}$} & \multirow{3}{*}{$\begin{array}{l}\text { No. of } \\
\text { Samples }\end{array}$} & \multirow{2}{*}{\multicolumn{3}{|c|}{$\left(10^{-3} \mathrm{mg} / 1\right)$}} & \multicolumn{2}{|c|}{$\left(10^{-3} \mathrm{mg} / 1\right)$} \\
\hline & & & & & & Minimum & Detectable \\
\hline & & & Average & Maximum & Minimum & Criteria & Limits \\
\hline
\end{tabular}

Cadmium (Cd)

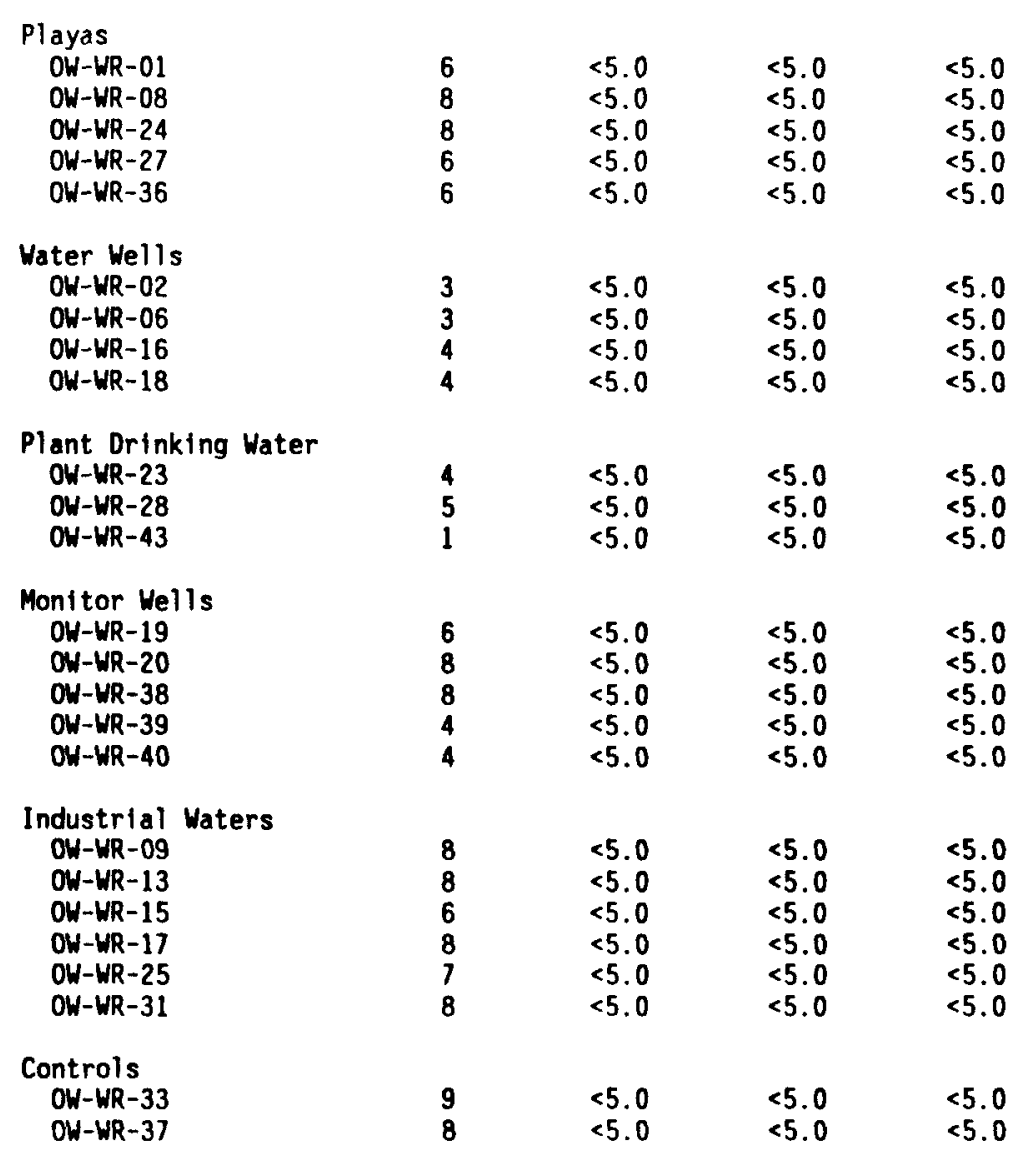

$50.0 \quad 5.0$

10.0

10.0 
Table XXXIV. Summary of Chemical Analysis for Water Pollutants for 1988 (Cont'd)

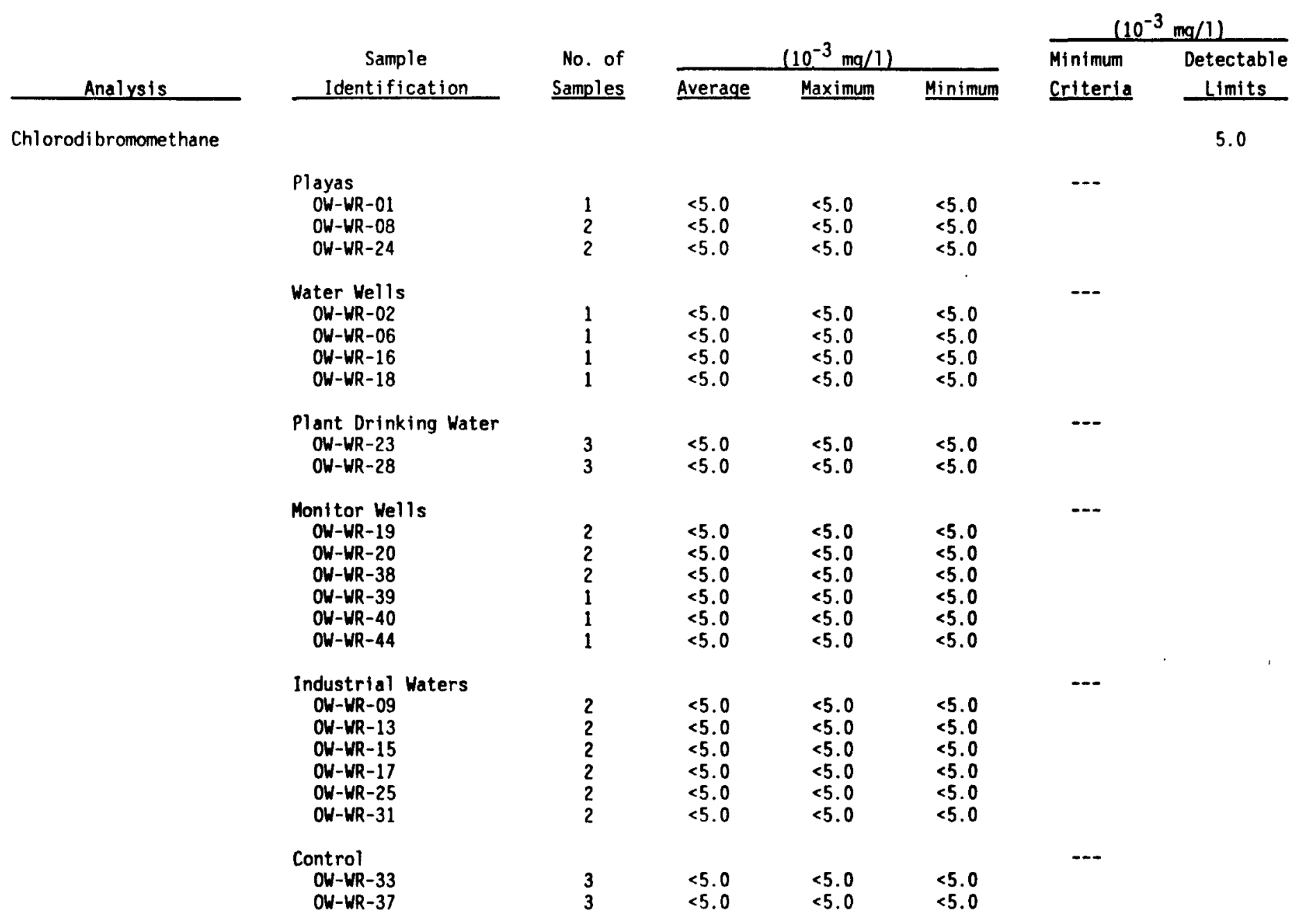


Table XXXIV. Sumary of Chemical Analysis for Water Pollutants for 1988 (Cont 'd)

\begin{tabular}{|c|c|c|c|c|c|c|c|}
\hline \multirow[b]{3}{*}{ Analysis } & \multirow{3}{*}{$\begin{array}{c}\text { Sample } \\
\text { Identification }\end{array}$} & \multirow{3}{*}{$\begin{array}{l}\text { No. of } \\
\text { Samples } \\
\end{array}$} & \multirow{2}{*}{\multicolumn{3}{|c|}{$\left(10^{-3} \mathrm{mg} / 1\right)$}} & \multicolumn{2}{|c|}{$\left(10^{-3} \mathrm{mg} / 1\right)$} \\
\hline & & & & & & \multirow{2}{*}{$\begin{array}{l}\text { Minimum } \\
\text { Criteria }\end{array}$} & \multirow{2}{*}{$\begin{array}{l}\text { Detectable } \\
\text { Limits } \\
\end{array}$} \\
\hline & & & Average & Maximum & Minimum & & \\
\hline & $\begin{array}{l}\text { Playas } \\
\text { OW-WR-01 } \\
\text { OW-WR-08 } \\
\text { OW-WR-24 }\end{array}$ & $\begin{array}{l}1 \\
2 \\
2\end{array}$ & $\begin{array}{l}<5.0 \\
<5.0 \\
<5.0\end{array}$ & $\begin{array}{l}<5.0 \\
<5.0 \\
<5.0\end{array}$ & $\begin{array}{l}<5.0 \\
<5.0 \\
<5.0\end{array}$ & $\cdots$ & \\
\hline & $\begin{array}{c}\text { Water We11s } \\
\text { OW-WR-02 } \\
\text { OW-WR-06 } \\
\text { OW-WR-16 } \\
\text { OW-WR-18 }\end{array}$ & $\begin{array}{l}1 \\
1 \\
1 \\
1\end{array}$ & $\begin{array}{l}<5.0 \\
<5.0 \\
<5.0 \\
<5.0\end{array}$ & $\begin{array}{l}<5.0 \\
<5.0 \\
<5.0 \\
<5.0\end{array}$ & $\begin{array}{l}<5.0 \\
<5.0 \\
<5.0 \\
<5.0\end{array}$ & --- & \\
\hline & $\begin{array}{l}\text { Plant Drinking Water } \\
\text { OW-WR-23 } \\
\text { OW-WR-28 }\end{array}$ & $\begin{array}{l}3 \\
3\end{array}$ & $\begin{array}{l}<5.0 \\
<5.0\end{array}$ & $\begin{array}{l}<5.0 \\
<5.0\end{array}$ & $\begin{array}{l}<5.0 \\
<5.0\end{array}$ & --- & \\
\hline & $\begin{array}{l}\text { Industrial Waters } \\
\text { OW-WR-09 } \\
\text { OW-WR-13 } \\
\text { OW-WR-15 } \\
\text { OW-WR-17 } \\
\text { OW-WR-25 } \\
\text { OW-WR-31 }\end{array}$ & $\begin{array}{l}2 \\
2 \\
2 \\
2 \\
2 \\
2\end{array}$ & $\begin{array}{l}<5.0 \\
<5.0 \\
<5.0 \\
<5.0 \\
<5.0 \\
<5.0\end{array}$ & $\begin{array}{l}<5.0 \\
<5.0 \\
<5.0 \\
<5.0 \\
<5.0 \\
<5.0\end{array}$ & $\begin{array}{l}<5.0 \\
<5.0 \\
<5.0 \\
<5.0 \\
<5.0 \\
<5.0\end{array}$ & $\cdots$ & \\
\hline
\end{tabular}


Table XXXIV. Summary of Chemical Analysis for Water Pollutants for 1988 (Cont'd)

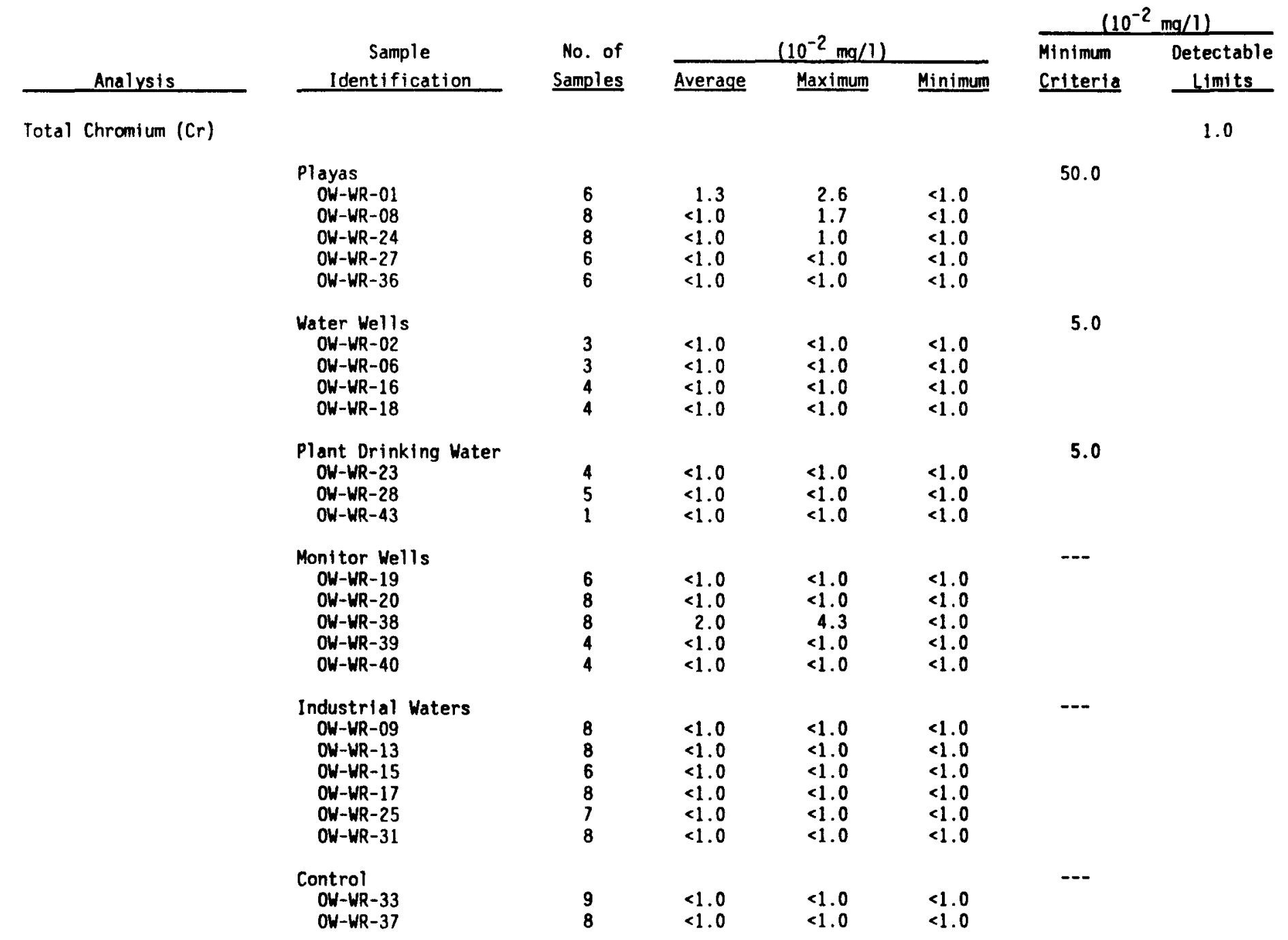


Table XXXIV. Summary of Chemical Analysis for Water Pollutants for 1988 (Cont 'd)

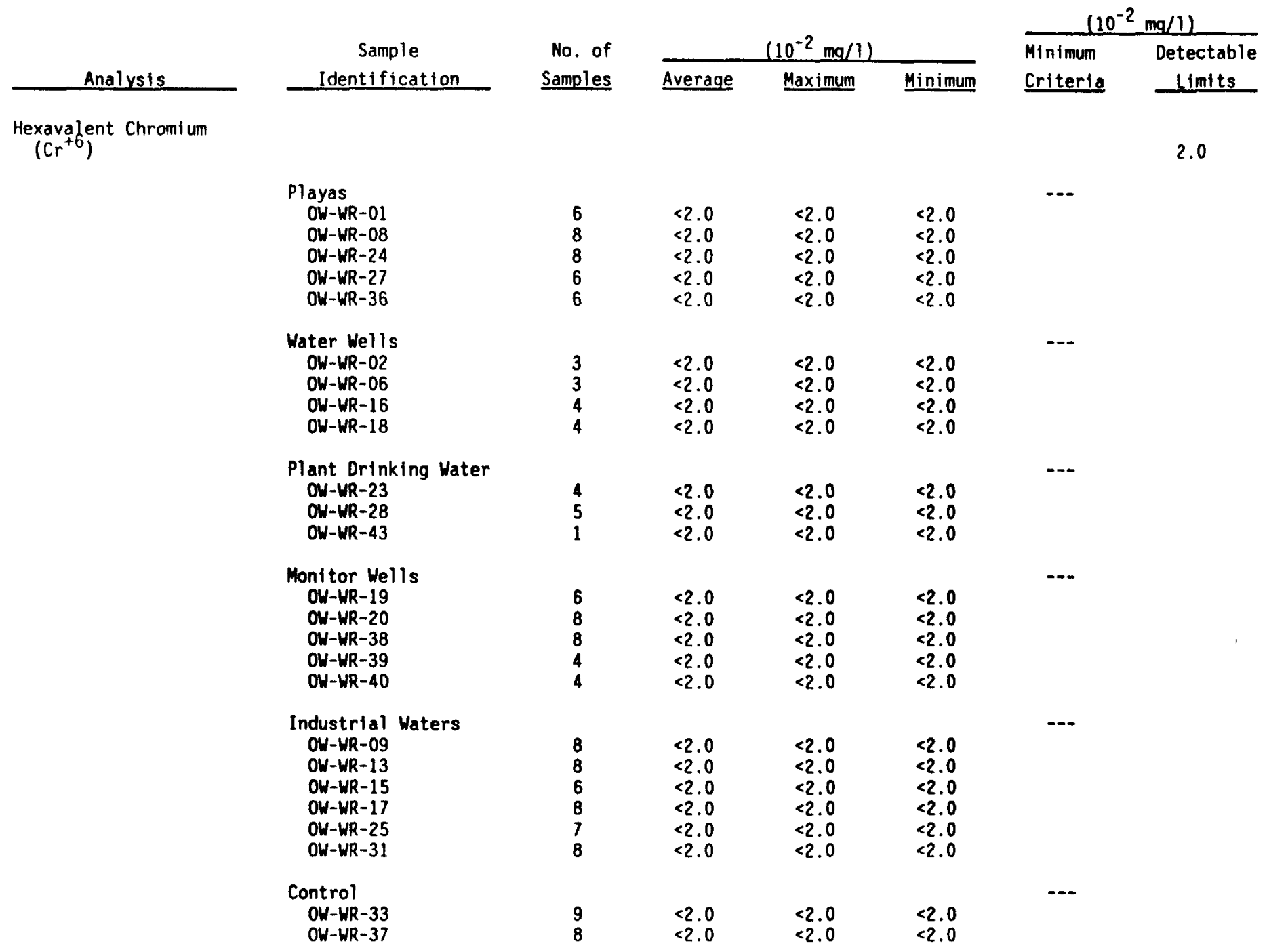


Table XXXIV. Summary of Chemical Analysis for Water Pollutants for 1988 (Cont'd)

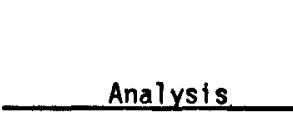

Copper $(\mathrm{Cu})$
Sample

Identification

$$
\begin{aligned}
& \text { Playas } \\
& \text { OW-WR-01 } \\
& \text { OW-WR-08 } \\
& \text { OW-WR-24 } \\
& \text { OW-WR-27 } \\
& \text { OW-WR-36 } \\
& \text { Water We11s } \\
& \text { OW-WR-02 } \\
& \text { OW-WR-06 } \\
& \text { OW-WR-16 } \\
& \text { OW-WR-18 }
\end{aligned}
$$

OW-WR-18

Plant Drinking Water OW-WR-23

OW-WR-28

OW-WR-43

Monitor Wells

OW-WR-19

OW-WR-20

OW-WR-38

OW-WR-39

OW-WR-40

Industrial Waters

OW-UR-09

OW-WR-13

OW-WR-15

OW-WR-17

OW-WR-25

OW-WR-31

\section{Control}

OW-WR-33

$$
\begin{aligned}
& 2 \\
& 2 \\
& 3 \\
& 3
\end{aligned}
$$

No. of

Samples

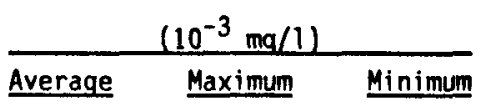

Maximum Minimum

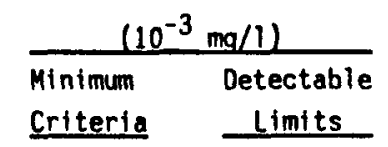

5.0

$\begin{array}{rrrr}5 & 16.8 & 33.0 & 8.0 \\ 6 & 6.8 & 10.0 & <5.0 \\ 6 & 8.3 & 20.0 & <.0 \\ 6 & 6.1 & 9.0 & <5.0 \\ 6 & 8.1 & 18.0 & <5.0\end{array}$$$
\begin{array}{r}
5 . \\
8 . \\
18 . \\
15 .
\end{array}
$$$$
<5.0
$$$$
\begin{aligned}
& 5.0<5.0 \\
& 11.0<5.0
\end{aligned}
$$$$
\begin{array}{lll}
8.0 & 40.0 & <5.0 \\
18.3 & 31.0 & <5.0
\end{array}
$$

$\begin{array}{rrrr}2 & 120.5 & 160.0 & 81.0 \\ 3 & 7.3 & 9.0 & 5.0 \\ 1 & 10.0 & 10.0 & 10.0\end{array}$

$\begin{aligned} & 4 \\ & 6 \\ & 6\end{aligned} \quad<5.0<\begin{aligned} & 7.0 \\ & 3.0 \\ & 3 \\ & 3\end{aligned}$

$3<5.0<5.0<5.0$

$\begin{array}{rrrr}6 & & & \\ 6 & 5.1 & 6.0 & <5.0 \\ 4 & 118.8 & 680.0 & <5.0 \\ 6 & 7.2 & 10.0 & <5.0 \\ 5 & 22.3 & 42.0 & <5.0 \\ 6 & 5.2 & 6.0 & <5.0 \\ & 6.3 & 9.0 & <5.0\end{array}$

$\begin{array}{rrrr}7 & 7.2 & 20.0 & 5.0 \\ 6 & 19.8 & 33.0 & 11.0\end{array}$


Table XXX1V. Summary of Chemical Analysis for Water Pollutants for 1988 (Cont'd)

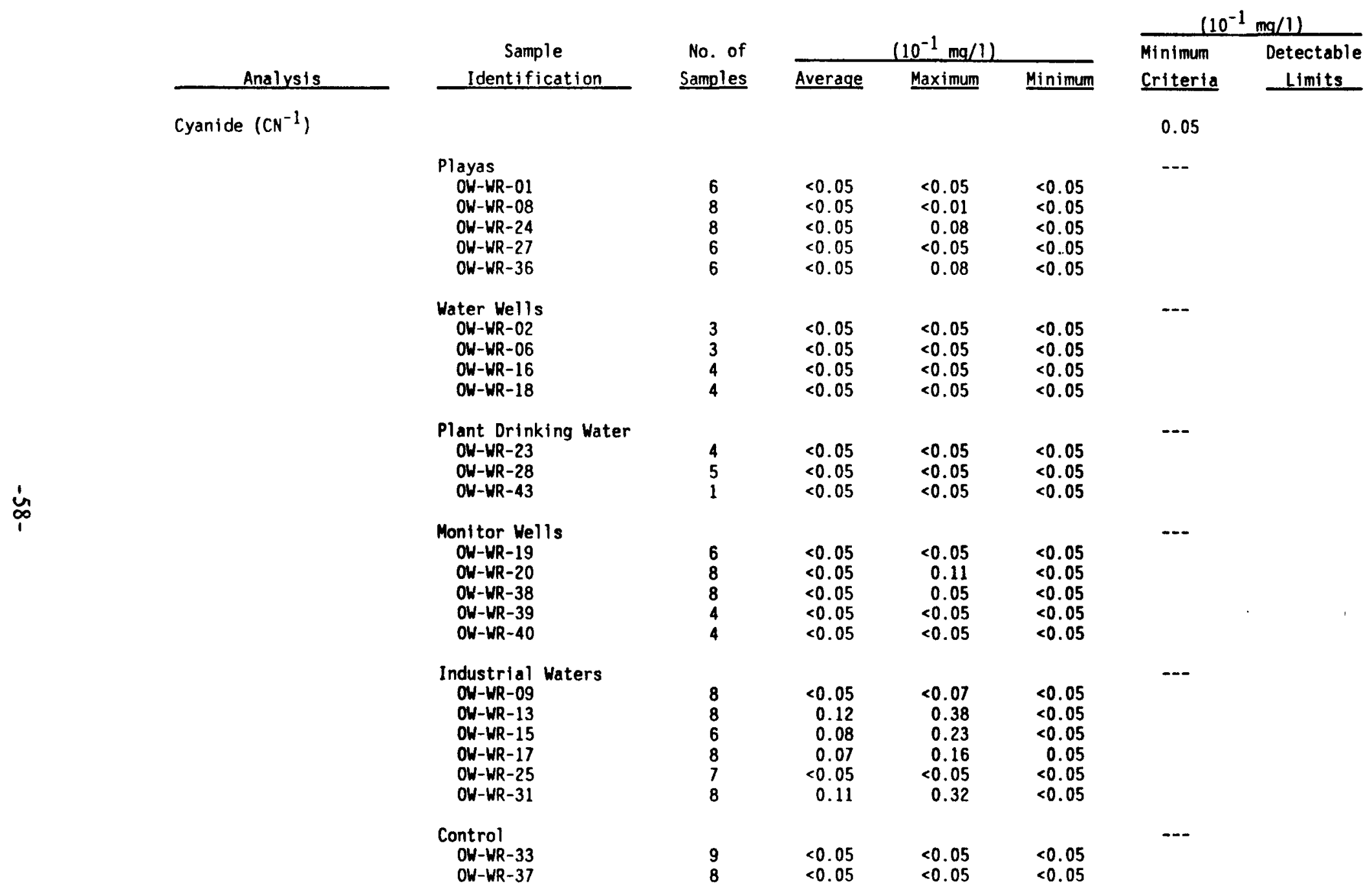


Table XXXIV. Summary of Chemical Analysis for Water Pollutants for 1988 (Cont 'd)

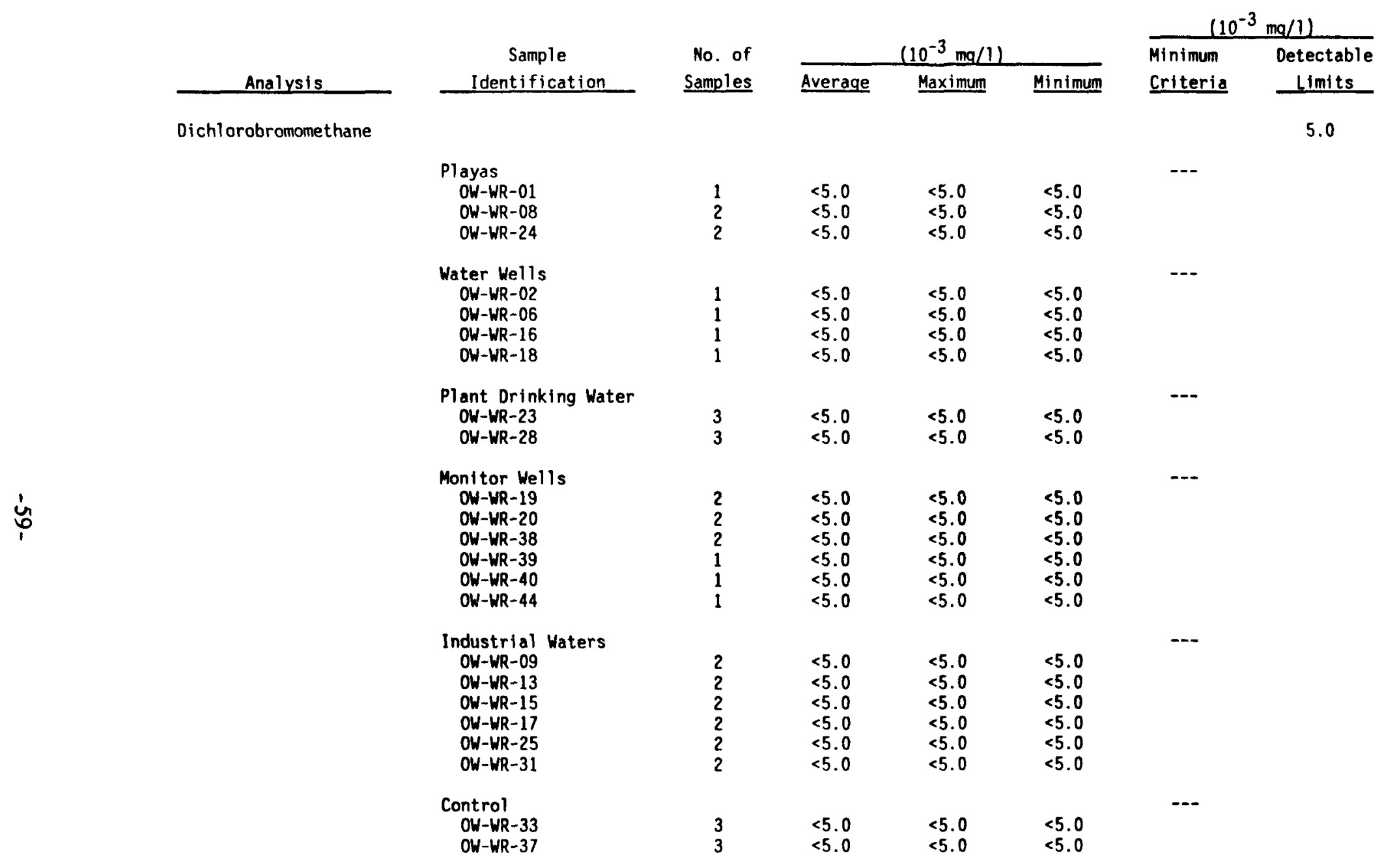


Table XXXIV. Summary of Chemical Analysis for Water Pollutants for 1988 (Cont'd)

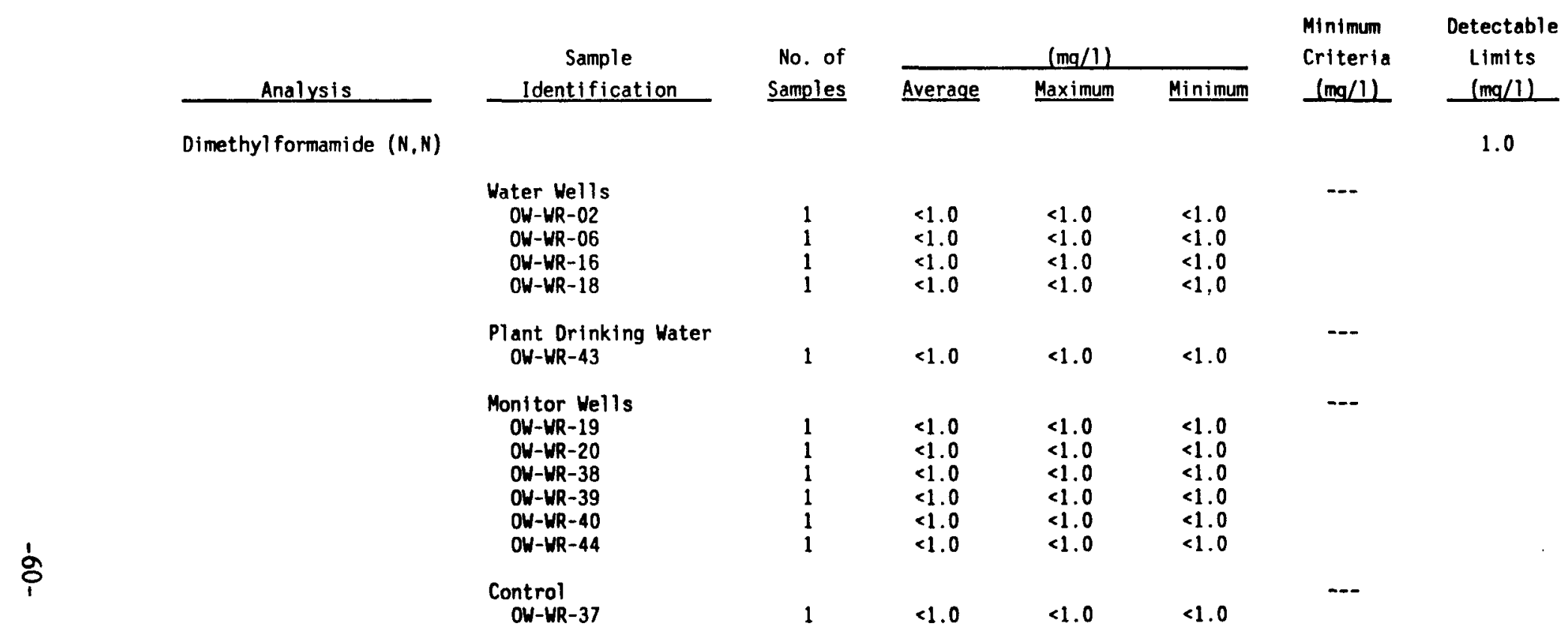


Table XXXIV. Summary of Chemical Analysis for Water Pollutants for 1988 (Cont'd)

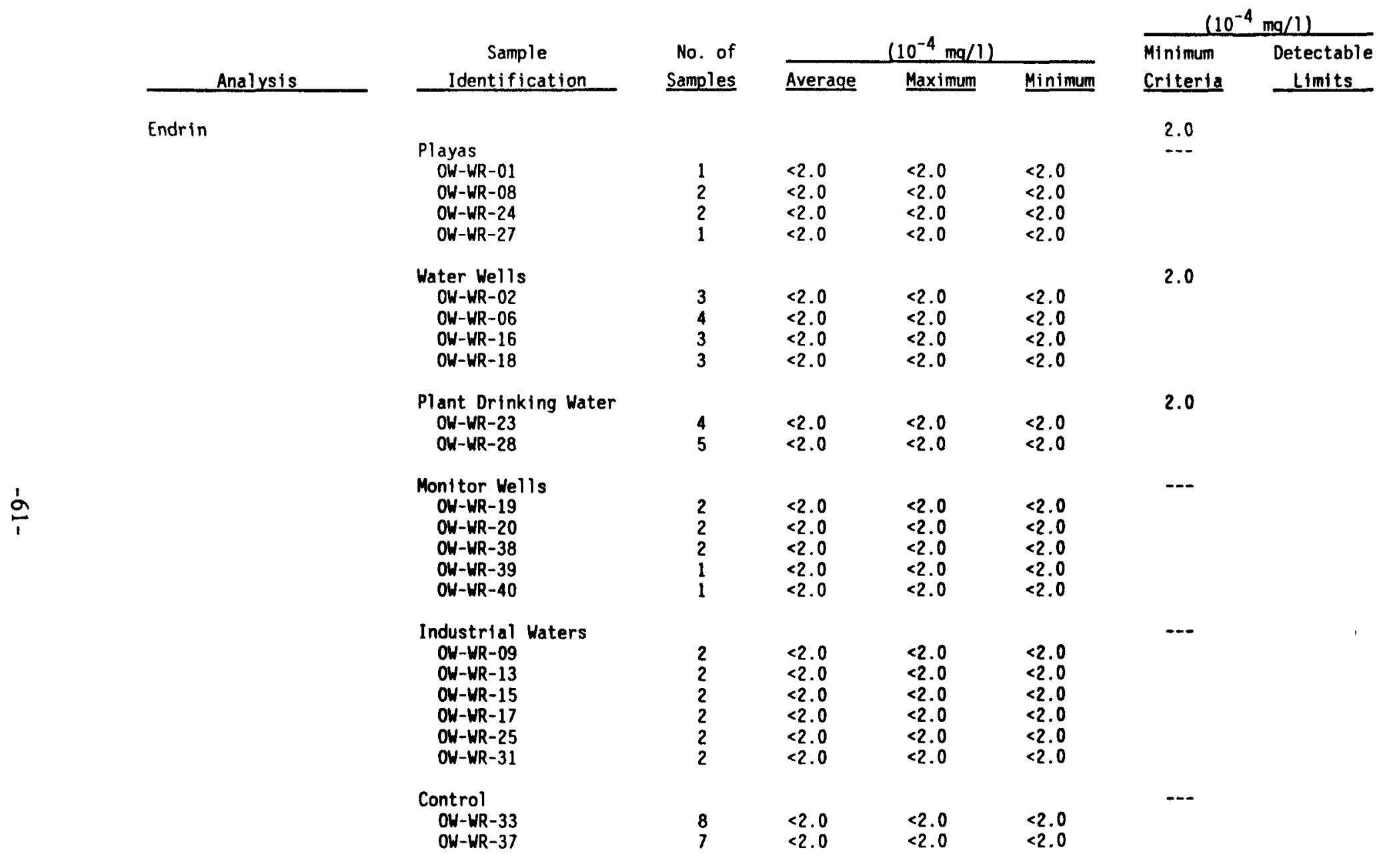


Table XXXIV. Summary of Chemical Analysis for Water Pollutants for 1988 (Cont 'd)

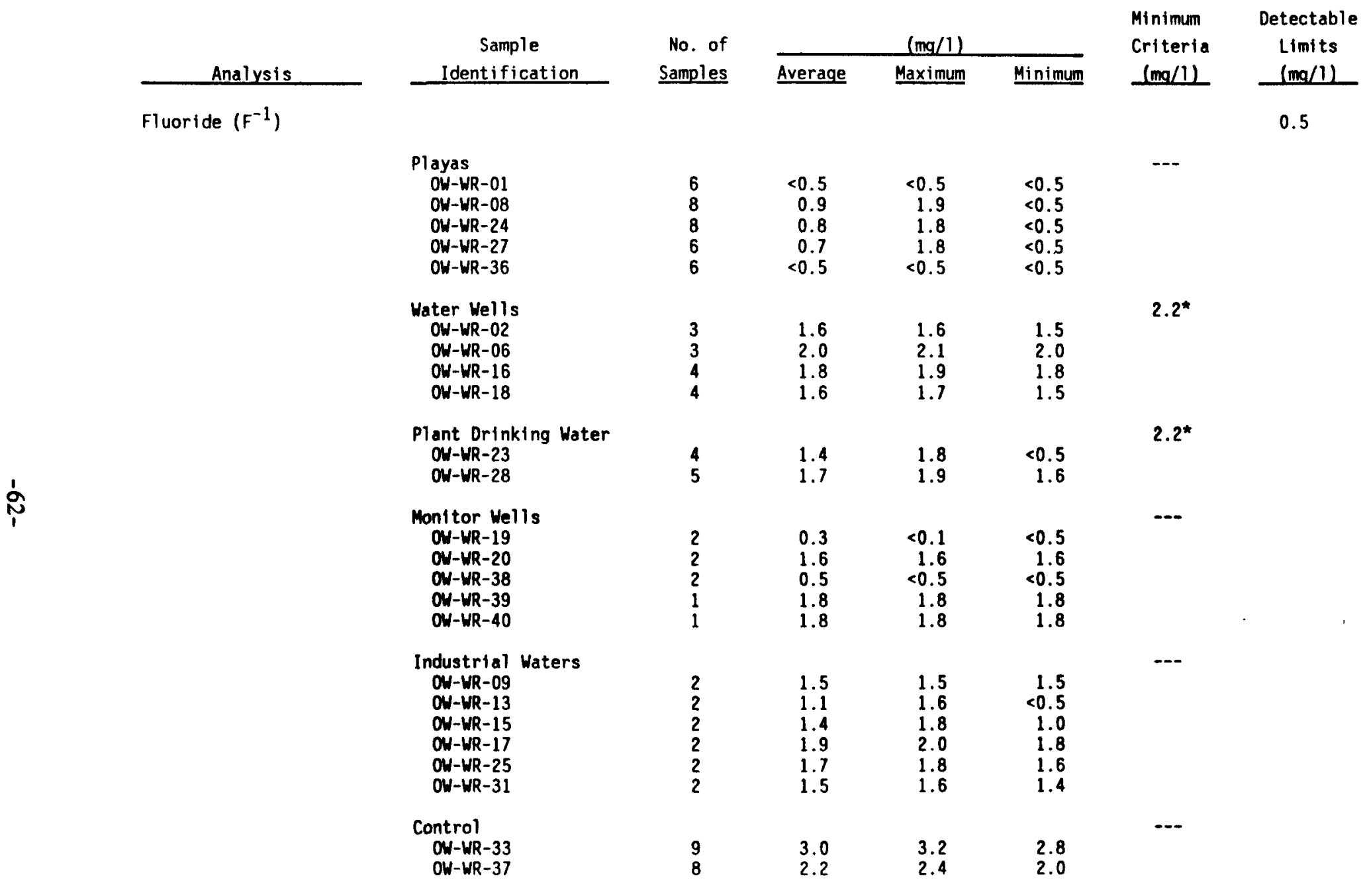


Table XXXIV. Summary of Chemical Analysis for Water Pollutants for 1988 (Cont 'd)

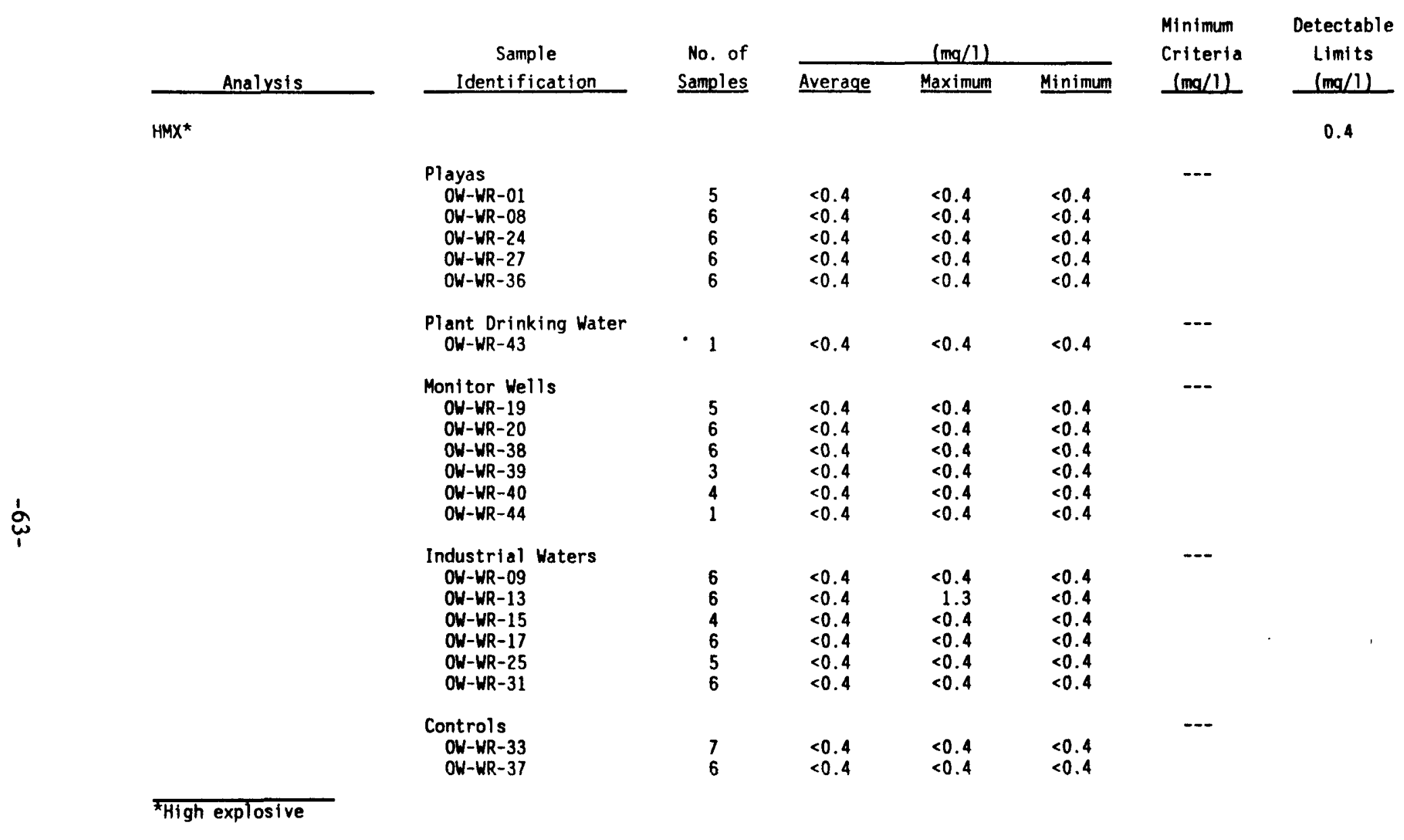


Table XXXIV. Summary of Chemical Analysis for Water Pollutants for 1988 (Cont'd)

\begin{tabular}{|c|c|c|c|c|c|c|c|}
\hline \multirow[b]{2}{*}{ Analysis } & \multirow{2}{*}{$\begin{array}{c}\text { Sample } \\
\text { Identification }\end{array}$} & \multirow{2}{*}{$\begin{array}{l}\text { No. of } \\
\text { Samples } \\
\end{array}$} & \multicolumn{3}{|c|}{$(\mathrm{mg} / 1)$} & \multirow{2}{*}{$\begin{array}{l}\text { Minimum } \\
\text { Criteria } \\
(\mathrm{mg} / 1)\end{array}$} & \multirow{2}{*}{$\begin{array}{c}\text { Detectable } \\
\text { Limits } \\
\text { (mg/1) } \\
\end{array}$} \\
\hline & & & Average & Maximum & Minimum & & \\
\hline Hydrocarbons* & & & & & & & 5.0 \\
\hline & $\begin{array}{c}\text { Water Wells } \\
\text { OW-WR-02 } \\
\text { OW-WR-06 } \\
\text { OW-WR-16 } \\
\text { OW-WR-18 }\end{array}$ & $\begin{array}{l}1 \\
1 \\
1 \\
1\end{array}$ & $\begin{array}{l}<5.0 \\
<5.0 \\
<5.0 \\
<5.0\end{array}$ & $\begin{array}{l}<5.0 \\
<5.0 \\
<5.0 \\
<5.0\end{array}$ & $\begin{array}{l}<5.0 \\
<5.0 \\
<5.0 \\
<5.0\end{array}$ & --- & \\
\hline & $\begin{array}{l}\text { Plant Drinking Water } \\
\text { OW-WR-19 } \\
\text { OW-WR-20 } \\
\text { OW-WR-38 } \\
\text { OW-WR-39 } \\
\text { OW-WR-40 } \\
\text { OW-WR-44 }\end{array}$ & $\begin{array}{l}1 \\
1 \\
1 \\
1 \\
1 \\
1\end{array}$ & $\begin{array}{l}<5.0 \\
<5.0 \\
<5.0 \\
<5.0 \\
<5.0 \\
<5.0\end{array}$ & $\begin{array}{l}<5.0 \\
<5.0 \\
<5.0 \\
<5.0 \\
<5.0 \\
<5.0\end{array}$ & $\begin{array}{l}<5.0 \\
<5.0 \\
<5.0 \\
<5.0 \\
<5.0 \\
<5.0\end{array}$ & --- & \\
\hline & $\begin{array}{l}\text { Control } \\
\text { OW-WR-37 }\end{array}$ & 1 & $<5.0$ & $<5.0$ & $<5.0$ & --- & \\
\hline
\end{tabular}

* Suspected underground gasoline tank leak prompted these samples to be taken. 
Table XXXIV. Surmary of Chemical Analysis for Water Pollutants for 1988 (Cont 'd)

\begin{tabular}{|c|c|c|c|c|c|c|c|}
\hline & Sample & No. of & & $(\mathrm{mq} / 1)$ & & $\begin{array}{l}\text { Minimum } \\
\text { Criteria }\end{array}$ & $\begin{array}{c}\text { Detectable } \\
\text { Limits }\end{array}$ \\
\hline Analysis & Identification & Samples & Average & Maximum & Minimum & $(\mathrm{mg} / 1)$ & $(\mathrm{mg} / 1)$ \\
\hline Iron (Fe) & & & & & & & 0.01 \\
\hline & $\begin{array}{l}\text { Playas } \\
\text { OW-WR-01 } \\
\text { OW-WR-08 } \\
\text { OW-WR-24 } \\
\text { OW-WR-27 } \\
\text { OW-WR-36 }\end{array}$ & $\begin{array}{l}5 \\
6 \\
6 \\
6 \\
6\end{array}$ & $\begin{array}{r}12.16 \\
4.28 \\
2.61 \\
1.96 \\
6.18\end{array}$ & $\begin{array}{r}24.00 \\
15.00 \\
6.20 \\
3.40 \\
12.00\end{array}$ & $\begin{array}{l}0.02 \\
0.70 \\
0.55 \\
0.38 \\
1.10\end{array}$ & --- & \\
\hline & $\begin{array}{c}\text { Water Wel ls } \\
\text { OW-WR-02 } \\
\text { OW-WR-06 } \\
\text { OW-WR-16 } \\
\text { OW-WR-18 }\end{array}$ & $\begin{array}{l}2 \\
2 \\
3 \\
3\end{array}$ & $\begin{array}{r}0.04 \\
0.07 \\
<0.01 \\
0.02\end{array}$ & $\begin{array}{r}0.05 \\
0.08 \\
<0.01 \\
0.03\end{array}$ & $\begin{array}{l}0.03 \\
0.06 \\
0.02 \\
0.02\end{array}$ & -- & \\
\hline & $\begin{array}{l}\text { Plant Drinking Water } \\
\text { OW-WR-23 } \\
\text { OW-WR-28 }\end{array}$ & $\begin{array}{l}2 \\
3\end{array}$ & $\begin{array}{r}0.02 \\
<0.01\end{array}$ & $\begin{array}{l}0.03 \\
0.02\end{array}$ & $\begin{array}{l}<0.01 \\
<0.01\end{array}$ & -- & \\
\hline$u_{1}^{s}$ & $\begin{array}{c}\text { Monitor Wells } \\
\text { OW-WR-19 } \\
\text { OW-WR-20 } \\
\text { OW-WR-38 } \\
\text { OW-WR-39 } \\
\text { OW-WR-40 }\end{array}$ & $\begin{array}{l}4 \\
6 \\
6 \\
3 \\
3\end{array}$ & $\begin{array}{l}3.85 \\
2.03 \\
0.81 \\
2.01 \\
0.03\end{array}$ & $\begin{array}{l}4.60 \\
3.30 \\
1.30 \\
4.60 \\
0.07\end{array}$ & $\begin{array}{r}2.80 \\
1.20 \\
0.40 \\
0.24 \\
<0.01\end{array}$ & $\cdots$ & \\
\hline & $\begin{array}{l}\text { Industrial Waters } \\
\text { OW-WR-09 } \\
\text { OW-WR-13 } \\
\text { OW-WR-15 } \\
\text { OW-WR-17 } \\
\text { OW-WR-25 } \\
\text { OW-WR-31 }\end{array}$ & $\begin{array}{l}6 \\
6 \\
4 \\
6 \\
5 \\
6\end{array}$ & $\begin{array}{l}0.87 \\
0.43 \\
0.51 \\
0.43 \\
1.52 \\
0.38\end{array}$ & $\begin{array}{l}4.50 \\
2.10 \\
1.10 \\
1.70 \\
5.30 \\
0.94\end{array}$ & $\begin{array}{l}0.60 \\
0.03 \\
0.11 \\
0.06 \\
0.21 \\
0.19\end{array}$ & --- & \\
\hline & $\begin{array}{l}\text { Control } \\
\text { OW-WR-33 } \\
\text { OW-WR-37 }\end{array}$ & $\begin{array}{l}7 \\
6\end{array}$ & $\begin{array}{r}<0.01 \\
0.02\end{array}$ & $\begin{array}{l}0.05 \\
0.04\end{array}$ & $\begin{array}{l}<0.01 \\
<0.01\end{array}$ & --- & \\
\hline
\end{tabular}


Table XXXIV. Summary of Chemical Analysis for Water Pollutants for 1988 (Cont 'd)

\begin{tabular}{|c|c|c|c|c|c|c|c|}
\hline \multirow[b]{3}{*}{ Analysis } & \multirow{3}{*}{$\begin{array}{c}\text { Sample } \\
\text { Identification }\end{array}$} & \multirow{3}{*}{$\begin{array}{l}\text { No. of } \\
\text { Samples }\end{array}$} & \multirow{2}{*}{\multicolumn{3}{|c|}{$\left(10^{-3} \mathrm{mg} / 1\right)$}} & \multicolumn{2}{|c|}{$\left(10^{-3} \mathrm{mg} / 1\right)$} \\
\hline & & & & & & \multirow{2}{*}{$\begin{array}{l}\text { Minimum } \\
\text { Criterla }\end{array}$} & \multirow{2}{*}{$\begin{array}{l}\text { Detectable } \\
\text { Limits } \\
\end{array}$} \\
\hline & & & Average & Maximum & Minimum & & \\
\hline \multirow[t]{7}{*}{ Lead $(\mathrm{Pb})$} & & & & & & & 5.0 \\
\hline & $\begin{array}{l}\text { Playas } \\
\text { OW-WR-01 } \\
\text { OW-WR-08 } \\
\text { OW-WR-24 } \\
\text { OW-WR-27 } \\
\text { OW-WR-36 }\end{array}$ & $\begin{array}{l}6 \\
8 \\
8 \\
6 \\
6\end{array}$ & $\begin{array}{r}28.8 \\
5.7 \\
8.8 \\
5.5 \\
6.6\end{array}$ & $\begin{array}{r}130.0 \\
8.0 \\
28.0 \\
8.0 \\
13.0\end{array}$ & $\begin{array}{l}<5.0 \\
<5.0 \\
<5.0 \\
<5.0 \\
<5.0\end{array}$ & 500 & \\
\hline & $\begin{array}{c}\text { Water Wells } \\
\text { OW-WR-02 } \\
\text { OW-WR-06 } \\
\text { OW-WR-16 } \\
\text { OW-WR-18 }\end{array}$ & $\begin{array}{l}3 \\
3 \\
4 \\
4\end{array}$ & $\begin{array}{r}<5.0 \\
<5.0 \\
\\
6.7 \\
8.7\end{array}$ & $\begin{array}{r}5.0 \\
<\quad 5.0 \\
12.0 \\
20.0\end{array}$ & $\begin{array}{l}<5.0 \\
<5.0 \\
<5.0 \\
<5.0\end{array}$ & 50 & \\
\hline & $\begin{array}{l}\text { Plant Drinking Water } \\
\text { OW-WR-23 } \\
\text { OW-WR-28 } \\
\text { OW-WR-43 }\end{array}$ & $\begin{array}{l}4 \\
5 \\
1\end{array}$ & $\begin{array}{l}<5.0 \\
<5.0 \\
<5.0\end{array}$ & $\begin{array}{l}<\quad 5.0 \\
<\quad 5.0 \\
<\quad 5.0\end{array}$ & $\begin{array}{l}<5.0 \\
<5.0 \\
<5.0\end{array}$ & 50 & \\
\hline & $\begin{array}{c}\text { Monitor Wells } \\
\text { OW-WR-19 } \\
\text { OW-WR-20 } \\
\text { OW-WR-38 } \\
\text { OW-WR-39 } \\
\text { OW-WR-40 }\end{array}$ & $\begin{array}{l}6 \\
8 \\
8 \\
4 \\
4\end{array}$ & $\begin{array}{r}6.5 \\
<5.0 \\
<5.0 \\
92.5 \\
<5.5\end{array}$ & $\begin{array}{r}14.0 \\
<\quad 5.0 \\
<\quad 5.0 \\
320.0 \\
7.0\end{array}$ & $\begin{array}{l}<5.0 \\
<5.0 \\
<5.0 \\
<5.0 \\
<5.0\end{array}$ & --- & , \\
\hline & $\begin{array}{l}\text { Industrial Waters } \\
\text { OW-WR-09 } \\
\text { OW-WR-13 } \\
\text { OW-WR-15 } \\
\text { OW-WR-17 } \\
\text { OW-WR-25 } \\
\text { OW-WR-31 }\end{array}$ & $\begin{array}{l}8 \\
8 \\
6 \\
8 \\
7 \\
8\end{array}$ & $\begin{aligned}< & 5.0 \\
& 6.0 \\
< & 5.0 \\
< & 5.0 \\
< & 5.0 \\
< & 5.0\end{aligned}$ & $\begin{array}{l}<5.0 \\
13.0 \\
<\quad 5.0 \\
<\quad 5.0 \\
<\quad 5.0 \\
<\quad 5.0\end{array}$ & $\begin{array}{l}<5.0 \\
<5.0 \\
<5.0 \\
<5.0 \\
<5.0 \\
<5.0\end{array}$ & --- & \\
\hline & $\begin{array}{l}\text { Control } \\
\text { OW-WR-33 } \\
\text { OW-WR-37 }\end{array}$ & $\begin{array}{l}9 \\
8\end{array}$ & $\begin{array}{r}5.0 \\
13.2\end{array}$ & $\begin{array}{r}5.0 \\
51.0\end{array}$ & $\begin{array}{l}<5.0 \\
<5.0\end{array}$ & -- & \\
\hline
\end{tabular}


Table XXXIV. Summary of Chemical Analysis for Water Pollutants for 1988 (Cont'd)

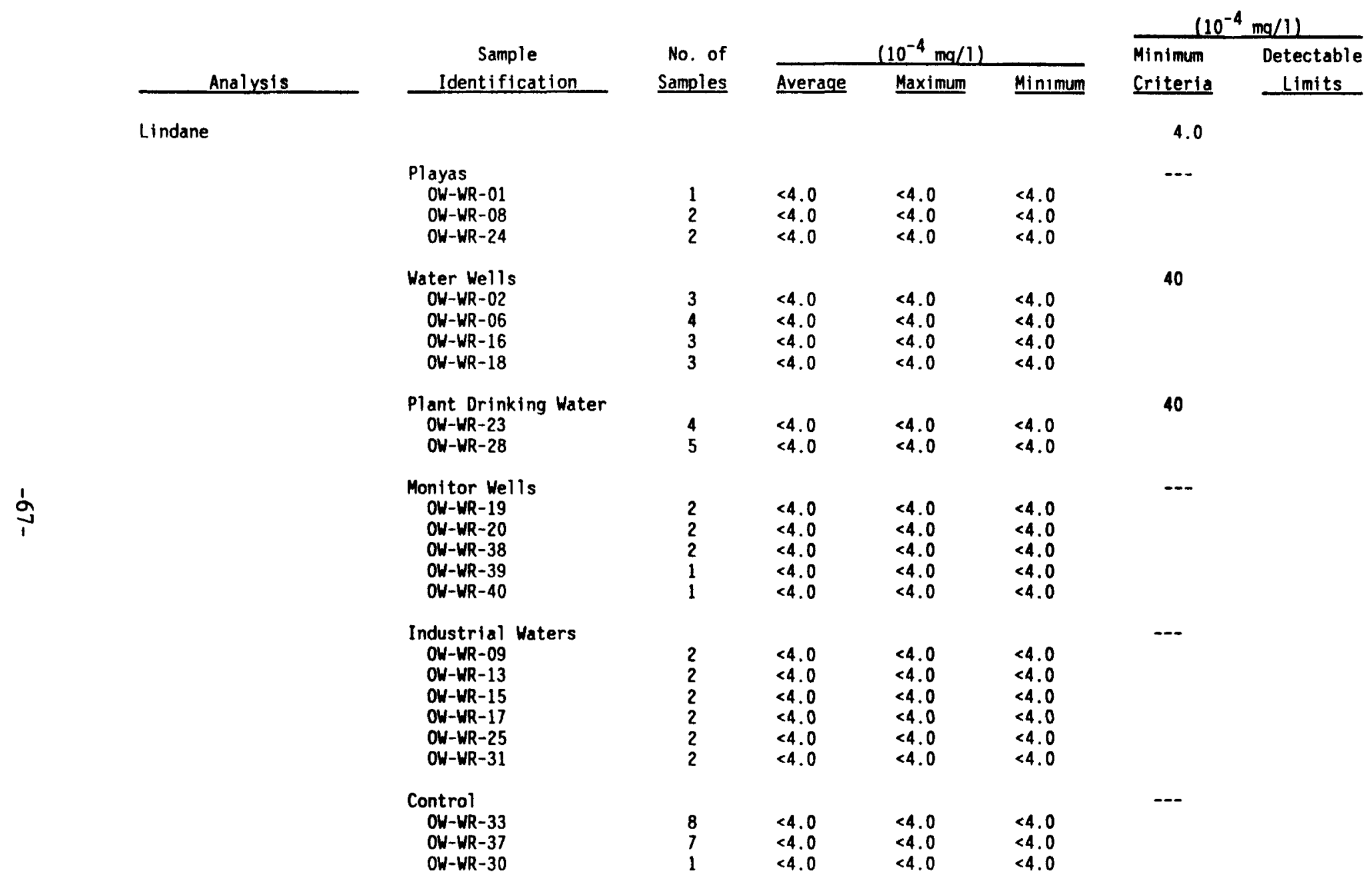


Table XXXIV. Summary of Chemical Analysis for Water Pollutants for 1988 (Cont'd)

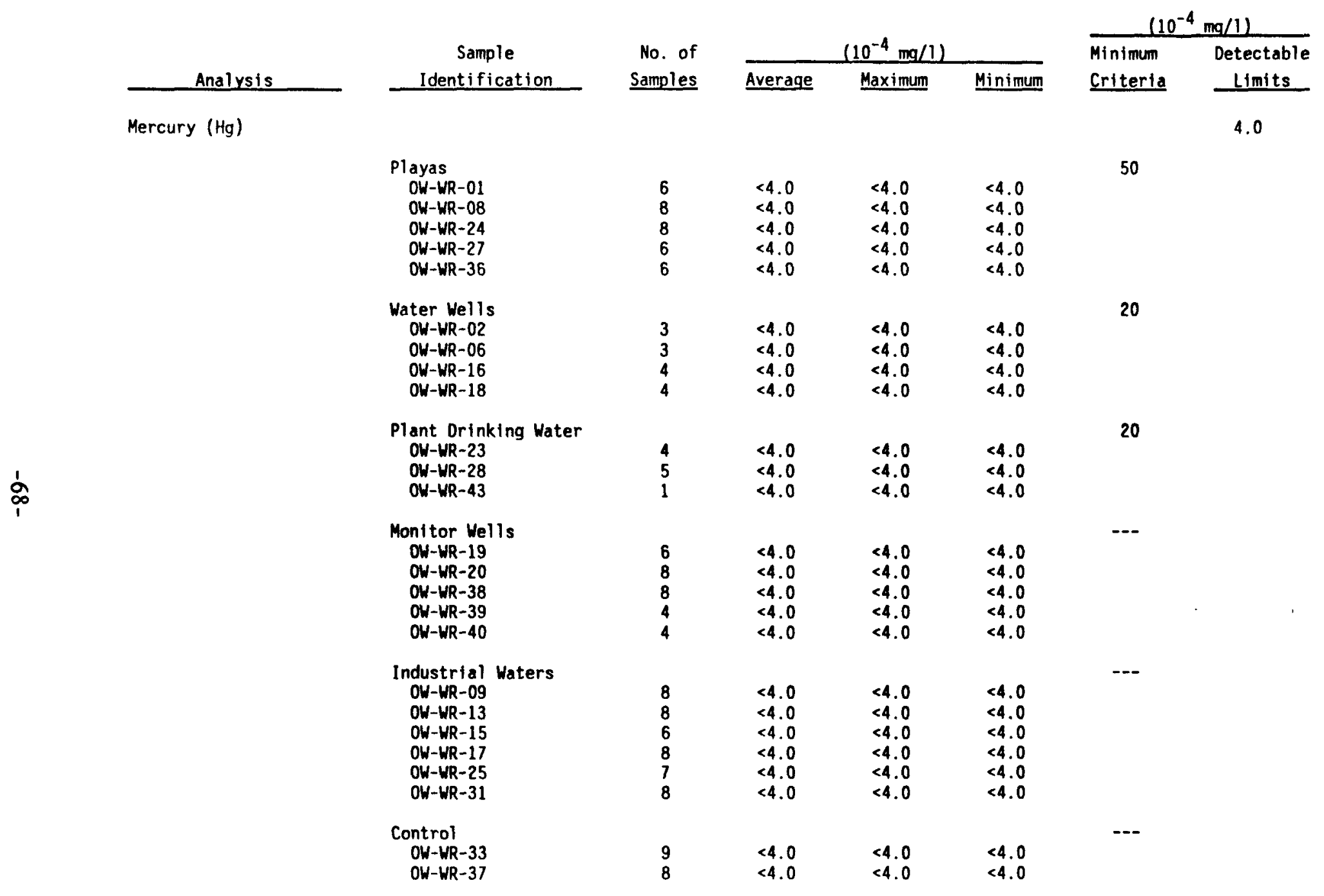


Table XXXIV. Summary of Chemical Analysis for Water Pollutants for 1988 (Cont'd)

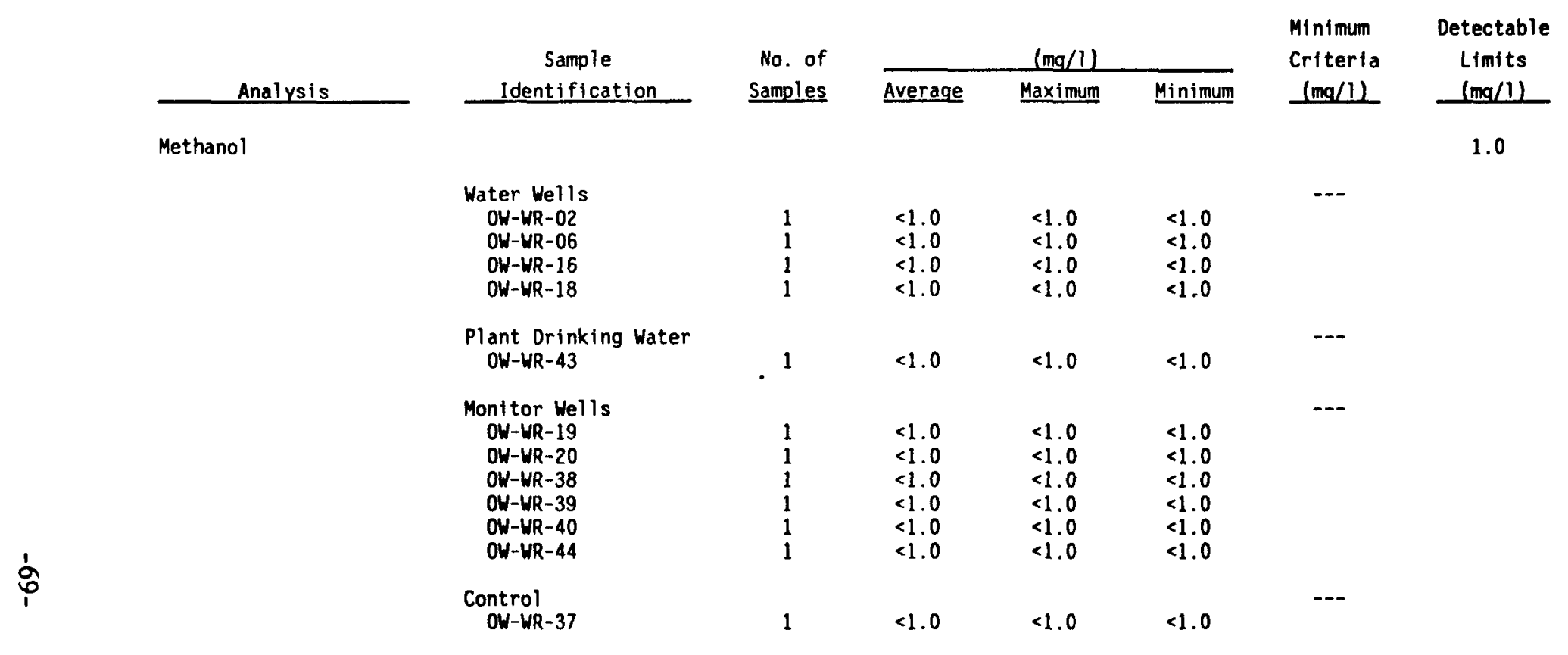


Table XXXIV. Summary of Chemical Analysis for Water Pollutants for 1988 (Cont'd)

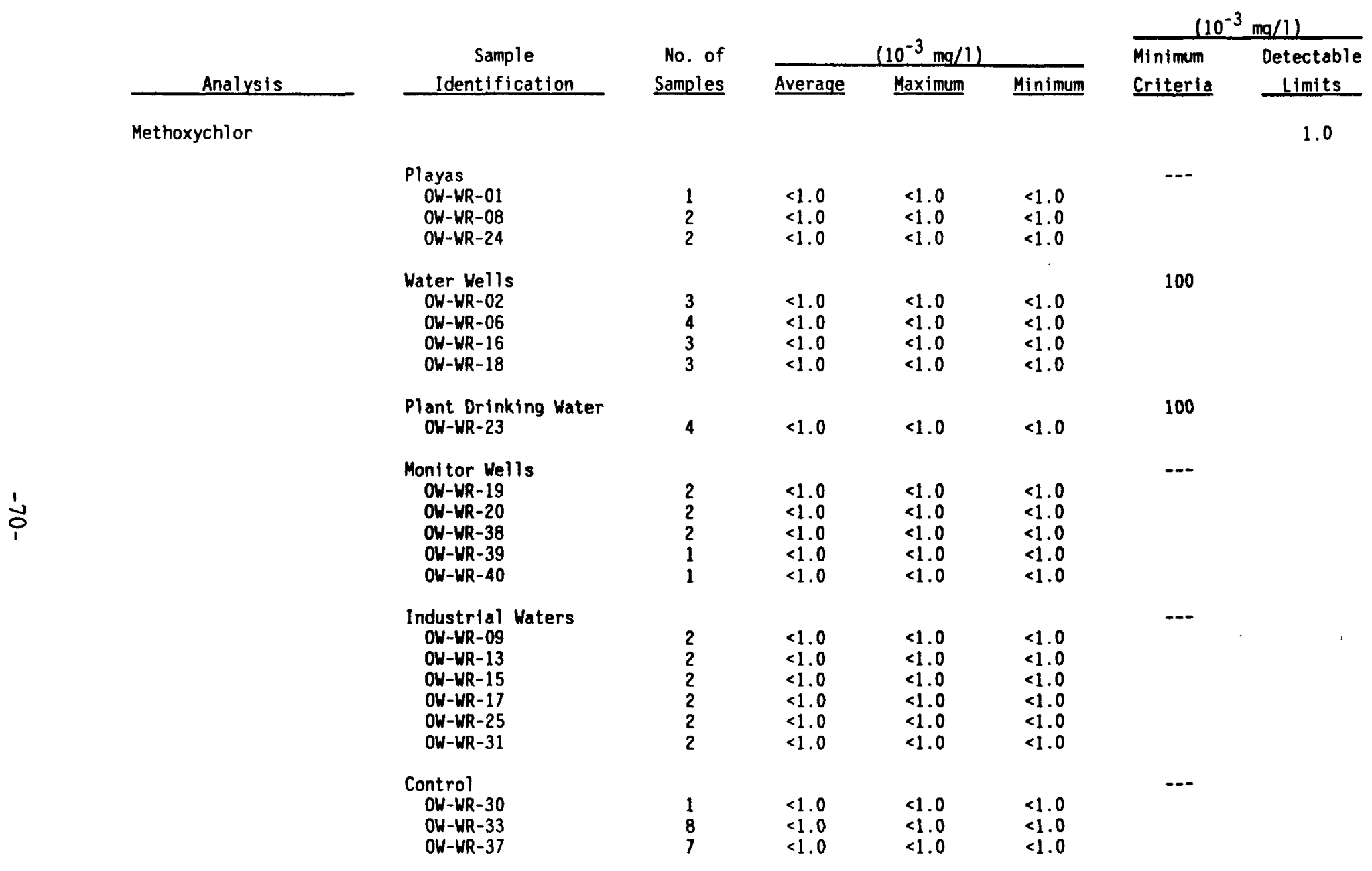


Table XXXIV. Surmary of Chemical Analysis for Water Pollutants for 1988 (Cont'd)

\begin{tabular}{|c|c|c|c|c|c|c|c|}
\hline \multirow[b]{2}{*}{ Analysis } & Sample & No. of & \multicolumn{3}{|c|}{$(\mathrm{mg} / 1)$} & \multirow{2}{*}{$\begin{array}{l}\text { Minimum } \\
\text { Criteria } \\
(\mathrm{mg} / 1) \\
\end{array}$} & \multirow{2}{*}{$\begin{array}{c}\text { Detectable } \\
\text { Limits } \\
\text { (mg/1) } \\
\end{array}$} \\
\hline & Identification & Samples & Average & Maximum & Minimum & & \\
\hline Methyl Ethyl Ketone & & & & & & & 1.0 \\
\hline & $\begin{array}{c}\text { Water Wells } \\
\text { OW-WR-02 } \\
\text { OW-WR-06 } \\
\text { OW-WR-16 } \\
\text { OW-WR-18 }\end{array}$ & $\begin{array}{l}1 \\
1 \\
1 \\
1\end{array}$ & $\begin{array}{l}<1.0 \\
<1.0 \\
<1.0 \\
<1.0\end{array}$ & $\begin{array}{l}<1.0 \\
<1.0 \\
<1.0 \\
<1.0\end{array}$ & $\begin{array}{l}<1.0 \\
<1.0 \\
<1.0 \\
<1.0\end{array}$ & --- & \\
\hline & $\begin{array}{l}\text { Plant Orinking Water } \\
\text { OW-WR-43 }\end{array}$ & 1 & $<1.0$ & $<1.0$ & $<1.0$ & --- & \\
\hline 1 & $\begin{array}{c}\text { Monitor Wells } \\
\text { OW-WR-19 } \\
\text { OW-WR-20 } \\
\text { OW-WR-38 } \\
\text { OW-WR-39 } \\
\text { OW-WR-40 } \\
\text { OW-WR-44 }\end{array}$ & $\begin{array}{l}1 \\
1 \\
1 \\
1 \\
1 \\
1\end{array}$ & $\begin{array}{l}<1.0 \\
<1.0 \\
<1.0 \\
<1.0 \\
<1.0 \\
<1.0\end{array}$ & $\begin{array}{l}<1.0 \\
<1.0 \\
<1.0 \\
<1.0 \\
<1.0 \\
<1.0\end{array}$ & $\begin{array}{l}<1.0 \\
<1.0 \\
<1.0 \\
<1.0 \\
<1.0 \\
<1.0\end{array}$ & -- & \\
\hline I & $\begin{array}{l}\text { Control } \\
\text { OW-WR-37 }\end{array}$ & 1 & $<1.0$ & $<1.0$ & $<1.0$ & --- & \\
\hline
\end{tabular}


Table Xxxiv. Summary of Chemical Analysis for Water Pollutants for 1988 (Cont'd)

\begin{tabular}{|c|c|c|c|c|c|c|c|}
\hline \multirow[b]{2}{*}{ Analysis } & \multirow{2}{*}{$\begin{array}{c}\text { Sample } \\
\text { Identification }\end{array}$} & \multirow{2}{*}{$\begin{array}{r}\text { No. of } \\
\text { Samples } \\
\end{array}$} & \multicolumn{3}{|c|}{$(\mathrm{mg} / 1)$} & \multirow{2}{*}{$\begin{array}{l}\text { Minimum } \\
\text { Criteria } \\
(\mathrm{mg} / 1) \\
\end{array}$} & \multirow{2}{*}{$\begin{array}{c}\text { Detectable } \\
\text { Limits } \\
(\mathrm{mg} / 1) \\
\end{array}$} \\
\hline & & & Average & Maximum & Minimum & & \\
\hline \multirow[t]{7}{*}{$\begin{array}{l}\text { Nitrate }\left(\mathrm{NO}_{3}\right)^{-1} \\
\text { Reported as } \mathrm{N} \\
\text { (Nitrogen) }\end{array}$} & & & & & & & 0.05 \\
\hline & $\begin{array}{l}\text { Playas } \\
\text { OW-WR-01 } \\
\text { OW-WR-08 } \\
\text { OW-WR-24 } \\
\text { OW-WR-27 } \\
\text { OW-WR-36 }\end{array}$ & $\begin{array}{l}6 \\
8 \\
8 \\
6 \\
6\end{array}$ & $\begin{array}{l}0.53 \\
0.20 \\
0.18 \\
0.06 \\
0.12\end{array}$ & $\begin{array}{l}1.20 \\
0.72 \\
0.49 \\
0.15 \\
0.24\end{array}$ & $\begin{array}{r}<0.05 \\
0.07 \\
0.25 \\
<0.05 \\
0.25\end{array}$ & $\cdots$ & \\
\hline & $\begin{array}{c}\text { Water Wells } \\
\text { OW-WR-02 } \\
\text { OW-WR-06 } \\
\text { OW-WR-16 } \\
\text { OW-WR-18 }\end{array}$ & $\begin{array}{l}3 \\
3 \\
4 \\
4\end{array}$ & $\begin{array}{l}1.20 \\
0.85 \\
1.45 \\
1.50\end{array}$ & $\begin{array}{l}1.30 \\
1.30 \\
1.60 \\
3.70\end{array}$ & $\begin{array}{r}1.10 \\
<0.05 \\
1.20 \\
0.13\end{array}$ & 10.0 & \\
\hline & $\begin{array}{l}\text { Plant Drinking Water } \\
\text { OW-WR-23 } \\
\text { OW-WR-28 } \\
\text { OW-WR-43 }\end{array}$ & $\begin{array}{l}4 \\
5 \\
1\end{array}$ & $\begin{array}{r}1.77 \\
1.28 \\
<0.05\end{array}$ & $\begin{array}{r}2.90 \\
1.50 \\
<0.05\end{array}$ & $\begin{array}{r}1.30 \\
1.10 \\
<0.05\end{array}$ & 10.0 & \\
\hline & $\begin{array}{c}\text { Monitor Wells } \\
\text { OW-WR-19 } \\
\text { OW-WR-20 } \\
\text { OW-WR-38 } \\
\text { OW-WR-39 } \\
\text { OW-WR-40 }\end{array}$ & $\begin{array}{l}6 \\
8 \\
8 \\
4 \\
4\end{array}$ & $\begin{array}{r}0.08 \\
<0.05 \\
2.26 \\
0.26 \\
<0.05\end{array}$ & $\begin{array}{r}0.08 \\
0.10 \\
2.90 \\
0.55 \\
<0.05\end{array}$ & $\begin{array}{r}0.25 \\
<0.05 \\
1.30 \\
<0.05 \\
<0.05\end{array}$ & -- & \\
\hline & $\begin{array}{c}\text { Industrial Waters } \\
\text { OW-WR-09 } \\
\text { OW-WR-13 } \\
\text { OW-WR-15 } \\
\text { OW-WR-17 } \\
\text { OW-WR-25 } \\
\text { OW-WR-31 }\end{array}$ & $\begin{array}{l}8 \\
8 \\
6 \\
8 \\
7 \\
8\end{array}$ & $\begin{array}{l}0.58 \\
1.26 \\
0.61 \\
0.66 \\
0.84 \\
0.15\end{array}$ & $\begin{array}{l}3.80 \\
2.60 \\
1.30 \\
1.90 \\
1.20 \\
0.57\end{array}$ & $\begin{array}{r}<0.05 \\
0.11 \\
<0.05 \\
<0.05 \\
0.20 \\
<0.05\end{array}$ & -- & \\
\hline & $\begin{array}{l}\text { Control } \\
\text { OW-WR-33 } \\
\text { OW-WR-37 }\end{array}$ & $\begin{array}{l}9 \\
8\end{array}$ & $\begin{array}{l}1.32 \\
0.88\end{array}$ & $\begin{array}{l}2.70 \\
1.20\end{array}$ & $\begin{array}{r}1.00 \\
<0.05\end{array}$ & -.- & \\
\hline
\end{tabular}


Table XXXIV. Summary of Chemical Analysis for Water Pollutants for 1988 (Cont 'd)

\begin{tabular}{|c|c|c|c|c|c|c|c|}
\hline \multirow[b]{2}{*}{ Analysis } & \multirow{2}{*}{$\begin{array}{c}\text { Sample } \\
\text { Identification }\end{array}$} & \multirow{2}{*}{$\begin{array}{l}\text { No. of } \\
\text { Samples }\end{array}$} & \multicolumn{3}{|c|}{$(\mathrm{mq} / 1)$} & \multirow{2}{*}{$\begin{array}{l}\text { Minimum } \\
\text { Criteria } \\
(\mathrm{mg} / 1) \\
\end{array}$} & \multirow{2}{*}{$\begin{array}{c}\text { Detectable } \\
\text { Limits } \\
(m g / 1) \\
\end{array}$} \\
\hline & & & Average & Maximum & Minimum & & \\
\hline $0 i 1$ and Grease & & & & & & & 3.0 \\
\hline & $\begin{array}{l}\text { Playas } \\
\text { OW-WR-01 } \\
\text { OW-WR-08 } \\
\text { OW-WR-24 } \\
\text { OW-WR-27 } \\
\text { OW-WR-36 }\end{array}$ & $\begin{array}{l}6 \\
8 \\
8 \\
6 \\
6\end{array}$ & $\begin{array}{l}<3.0 \\
<3.0 \\
<3.0 \\
<3.0 \\
<3.0\end{array}$ & $\begin{array}{l}<3.0 \\
<3.0 \\
<3.0 \\
<3.0 \\
<3.0\end{array}$ & $\begin{array}{l}<3.0 \\
<3.0 \\
<3.0 \\
<3.0 \\
<3.0\end{array}$ & -- & \\
\hline & $\begin{array}{c}\text { Water Wells } \\
\text { OW-WR-02 } \\
\text { OW-WR-06 } \\
\text { OW-WR-16 } \\
\text { OW-WR-18 }\end{array}$ & $\begin{array}{l}1 \\
1 \\
1 \\
1\end{array}$ & $\begin{array}{l}<3.0 \\
<3.0 \\
<3.0 \\
12.0\end{array}$ & $\begin{array}{l}<3.0 \\
<3.0 \\
<3.0 \\
12.0\end{array}$ & $\begin{array}{l}<3.0 \\
<3.0 \\
<3.0 \\
12.0\end{array}$ & --- & \\
\hline & $\begin{array}{l}\text { Plant Drinking Water } \\
\text { OW-WR-23 } \\
\text { OW-WR-28 }\end{array}$ & $\begin{array}{l}2 \\
2\end{array}$ & $\begin{array}{l}<3.0 \\
<3.0\end{array}$ & $\begin{array}{l}<3.0 \\
<3.0\end{array}$ & $\begin{array}{l}<3.0 \\
<3.0\end{array}$ & --- & \\
\hline & $\begin{array}{c}\text { Monit tor Wells } \\
\text { OW-WR-19 } \\
\text { OW-WR-20 } \\
\text { OW-WR-38 } \\
\text { OW-WR-39 } \\
\text { OW-WR-40 }\end{array}$ & $\begin{array}{l}2 \\
2 \\
2 \\
1 \\
1\end{array}$ & $\begin{array}{l}<3.0 \\
<3.0 \\
<3.0 \\
<3.0 \\
<3.0\end{array}$ & $\begin{array}{l}<3.0 \\
<3.0 \\
<3.0 \\
<3.0 \\
<3.0\end{array}$ & $\begin{array}{l}<3.0 \\
<3.0 \\
<3.0 \\
<3.0 \\
<3.0\end{array}$ & --- & \\
\hline & $\begin{array}{l}\text { Industrial Waters } \\
\text { OW-WR-09 } \\
\text { OW-WR-13 } \\
\text { OW-WR-15 } \\
\text { OW-WR-17 } \\
\text { OW-WR-25 } \\
\text { OW-WR-31 }\end{array}$ & $\begin{array}{l}8 \\
8 \\
6 \\
8 \\
7 \\
8\end{array}$ & $\begin{array}{l}<3.0 \\
<3.0 \\
<3.0 \\
<3.0 \\
<3.0 \\
<3.0\end{array}$ & $\begin{array}{l}<3.0 \\
<3.0 \\
<3.0 \\
<3.0 \\
<3.0 \\
<3.0\end{array}$ & $\begin{array}{l}<3.0 \\
<3.0 \\
<3.0 \\
<3.0 \\
<3.0 \\
<3.0\end{array}$ & -- & \\
\hline & $\begin{array}{l}\text { Control } \\
\text { OH-WR-33 } \\
\text { OW-WR-37 }\end{array}$ & $\begin{array}{l}9 \\
8\end{array}$ & $\begin{array}{l}<3.0 \\
<3.0\end{array}$ & $\begin{array}{l}<3.0 \\
<3.0\end{array}$ & $\begin{array}{l}<3.0 \\
<3.0\end{array}$ & $\cdots$ & \\
\hline
\end{tabular}


Table XXXIV. Summary of Chemical Analysis for Water Pollutants for 1988 (Cont 'd)

\begin{tabular}{|c|c|c|c|c|c|c|c|}
\hline \multirow[b]{2}{*}{ Analysis } & Sample & No. of & \multicolumn{3}{|c|}{$(\mathrm{mg} / 1)$} & \multirow{2}{*}{$\begin{array}{l}\text { Minimum } \\
\text { Criteria } \\
(\mathrm{mg} / 1) \\
\end{array}$} & \multirow{2}{*}{$\begin{array}{c}\text { Detectable } \\
\text { Limits } \\
(\mathrm{mq} / 1) \\
\end{array}$} \\
\hline & Identification & Samples & Average & Maximum & Minimum & & \\
\hline \multirow[t]{4}{*}{ PETN* } & & & & & & & 0.4 \\
\hline & $\begin{array}{l}\text { Playas } \\
\text { OW-WR-01 } \\
\text { OW-WR-08 }\end{array}$ & $\begin{array}{l}1 \\
5\end{array}$ & $\begin{array}{l}<0.4 \\
<0.4\end{array}$ & $\begin{array}{l}<0.4 \\
<0.4\end{array}$ & $\begin{array}{l}<0.4 \\
<0.4\end{array}$ & -- & \\
\hline & $\begin{array}{c}\text { Water Wells } \\
\text { OW-WR-02 } \\
\text { OW-WR-06 } \\
\text { OW-WR-16 } \\
\text { OW-WR-18 }\end{array}$ & $\begin{array}{l}2 \\
2 \\
3 \\
2\end{array}$ & $\begin{array}{l}<0.4 \\
<0.4 \\
<0.4 \\
<0.4\end{array}$ & $\begin{array}{l}<0.4 \\
<0.4 \\
<0.4 \\
<0.4\end{array}$ & $\begin{array}{l}<0.4 \\
<0.4 \\
<0.4 \\
<0.4\end{array}$ & --- & \\
\hline & $\begin{array}{l}\text { Plant Drinking Water } \\
\text { OW-WR-23 } \\
\text { OW-WR-28 }\end{array}$ & $\begin{array}{l}3 \\
5\end{array}$ & $\begin{array}{l}<0.4 \\
<0.4\end{array}$ & $\begin{array}{l}<0.4 \\
<0.4\end{array}$ & $\begin{array}{l}<0.4 \\
<0.4\end{array}$ & --- & \\
\hline \multirow[t]{3}{*}{$\frac{1}{f}$} & $\begin{array}{l}\text { Test Wells } \\
\text { OW-WR-19 } \\
\text { OW-WR-20 }\end{array}$ & $\begin{array}{l}5 \\
5\end{array}$ & $\begin{array}{l}<0.4 \\
<0.4\end{array}$ & $\begin{array}{r}0.4 \\
<0.4\end{array}$ & $\begin{array}{l}<0.4 \\
<0.4\end{array}$ & $\cdots$ & \\
\hline & $\begin{array}{l}\text { Industrial Waters } \\
\text { OW-WR-09 } \\
\text { OW-WR-13 } \\
\text { OW-WR-15 } \\
\text { OW-WR-17 } \\
\text { OW-WR-26 } \\
\text { OW-WR-31 }\end{array}$ & $\begin{array}{l}5 \\
5 \\
5 \\
4 \\
3 \\
4\end{array}$ & $\begin{array}{l}<0.4 \\
<0.4 \\
<0.4 \\
<0.4 \\
<0.4 \\
<0.4\end{array}$ & $\begin{array}{l}<0.4 \\
<0.4 \\
<0.4 \\
<0.4 \\
<0.4 \\
<0.4\end{array}$ & $\begin{array}{l}<0.4 \\
<0.4 \\
<0.4 \\
<0.4 \\
<0.4 \\
<0.4\end{array}$ & --- & , \\
\hline & $\begin{array}{l}\text { Control } \\
\text { OW-WR-33 }\end{array}$ & 5 & $<0.4$ & $<0.4$ & $<0.4$ & -- & \\
\hline
\end{tabular}


Table XxXIV. Summary of Chemical Analysis for Water Pollutants for 1988 (Cont'd)

\begin{tabular}{|c|c|c|c|c|c|c|c|}
\hline \multirow[b]{2}{*}{ Analysis } & \multirow{2}{*}{$\begin{array}{c}\text { Sample } \\
\text { Ident ification }\end{array}$} & \multirow{2}{*}{$\begin{array}{r}\text { No. of } \\
\text { Samples } \\
\end{array}$} & \multicolumn{3}{|c|}{$(\mathrm{mg} / 1)$} & \multirow{2}{*}{$\begin{array}{l}\text { Minimum } \\
\text { Criteria } \\
\text { (mg/l) }\end{array}$} & \multirow{2}{*}{$\begin{array}{c}\text { Detectable } \\
\text { Limits } \\
\text { (ma/1) }\end{array}$} \\
\hline & & & Averaqe & Maximum & Minimum & & \\
\hline \multirow{4}{*}{ Phenols } & $\begin{array}{l}\text { Playas } \\
\text { OW-WR-01 } \\
\text { OW-WR-08 } \\
\text { OW-WR-24 } \\
\text { OW-WR-27 } \\
\text { OW-WR-36 }\end{array}$ & $\begin{array}{l}3 \\
4 \\
4 \\
3 \\
3\end{array}$ & $\begin{array}{l}<0.02 \\
<0.02 \\
<0.02 \\
<0.02 \\
<0.02\end{array}$ & $\begin{array}{l}<0.02 \\
<0.02 \\
<0.02 \\
<0.02 \\
<0.02\end{array}$ & $\begin{array}{l}<0.02 \\
<0.02 \\
<0.02 \\
<0.02 \\
<0.02\end{array}$ & --- & \\
\hline & $\begin{array}{c}\text { Water Wells } \\
\text { OW-WR-02 } \\
\text { OW-WR-06 } \\
\text { OW-WR-16 } \\
\text { OW-WR-18 }\end{array}$ & $\begin{array}{r}1 \\
2 \\
3 \\
3\end{array}$ & $\begin{array}{l}<0.02 \\
<0.02 \\
<0.02 \\
<0.02\end{array}$ & $\begin{array}{l}<0.02 \\
<0.02 \\
<0.02 \\
<0.02\end{array}$ & $\begin{array}{l}<0.02 \\
<0.02 \\
<0.02 \\
<0.02\end{array}$ & --- & \\
\hline & $\begin{array}{l}\text { Monitor Wells } \\
\text { OW-WR-19 } \\
\text { OW-WR-20 } \\
\text { OW-WR-38 } \\
\text { OW-WR-39 } \\
\text { OW-WR-40 } \\
\text { OW-WR-44 }\end{array}$ & $\begin{array}{l}4 \\
4 \\
4 \\
3 \\
2 \\
1\end{array}$ & $\begin{array}{l}<0.02 \\
<0.02 \\
<0.02 \\
<0.02 \\
<0.02 \\
<0.02\end{array}$ & $\begin{array}{l}<0.02 \\
<0.02 \\
<0.02 \\
<0.02 \\
<0.02 \\
<0.02\end{array}$ & $\begin{array}{l}<0.02 \\
<0.02 \\
<0.02 \\
<0.02 \\
<0.02 \\
<0.02\end{array}$ & $\cdots$ & \\
\hline & $\begin{array}{l}\text { Industrial Waters } \\
\text { OW-WR-09 } \\
\text { OW-WR-13 } \\
\text { OW-WR-15 } \\
\text { OW-WR-17 } \\
\text { OW-WR-25 } \\
\text { OW-WR-31 }\end{array}$ & $\begin{array}{l}4 \\
4 \\
3 \\
4 \\
3 \\
4\end{array}$ & $\begin{array}{l}<0.02 \\
<0.02 \\
<0.02 \\
<0.02 \\
<0.02 \\
<0.02\end{array}$ & $\begin{array}{l}<0.02 \\
<0.02 \\
<0.02 \\
<0.02 \\
<0.02 \\
<0.02\end{array}$ & $\begin{array}{l}<0.02 \\
<0.02 \\
<0.02 \\
<0.02 \\
<0.02 \\
<0.02\end{array}$ & --- & \\
\hline
\end{tabular}


Table XXXIV. Surmary of Chemical Analysis for Water Pollutants for 1988 (Cont'd)

\begin{tabular}{|c|c|c|c|c|c|c|c|}
\hline \multirow[b]{2}{*}{ Analysis } & \multirow{2}{*}{$\begin{array}{c}\text { Sample } \\
\text { Identification }\end{array}$} & \multirow{2}{*}{$\begin{array}{c}\text { No. of } \\
\text { Samples } \\
\end{array}$} & \multicolumn{3}{|c|}{$(\mathrm{mq} / 1)$} & \multirow{2}{*}{$\begin{array}{l}\text { Minimum } \\
\text { Criteria } \\
(\mathrm{mg} / 1) \\
\end{array}$} & \multirow{2}{*}{$\begin{array}{c}\text { Detectable } \\
\text { Limits } \\
(\mathrm{mg} / 1) \\
\end{array}$} \\
\hline & & & Average & Maximum & Minimum & & \\
\hline Phosphate $\left(\mathrm{PO}_{4}\right)^{-3}$ & & & & & & & 0.02 \\
\hline & $\begin{array}{l}\text { Playas } \\
\text { OW-WR-01 } \\
\text { OW-WR-08 } \\
\text { OW-WR-24 } \\
\text { OW-WR-27 } \\
\text { OW-WR-36 }\end{array}$ & $\begin{array}{l}6 \\
8 \\
8 \\
6 \\
6\end{array}$ & $\begin{array}{l}0.21 \\
0.39 \\
0.27 \\
0.38 \\
0.47\end{array}$ & $\begin{array}{l}3.7 \\
0.11 \\
0.58 \\
0.64 \\
1.00\end{array}$ & $\begin{array}{l}1.0 \\
0.50 \\
0.11 \\
0.09 \\
0.12\end{array}$ & -- & \\
\hline & $\begin{array}{c}\text { Water We11s } \\
\text { OW-WR-02 } \\
\text { OW-WR-06 } \\
\text { OW-WR-16 } \\
\text { OW-WR-18 }\end{array}$ & $\begin{array}{l}1 \\
1 \\
1 \\
1\end{array}$ & $\begin{array}{r}<0.02 \\
<0.02 \\
0.04 \\
0.04\end{array}$ & $\begin{array}{r}<0.02 \\
<0.02 \\
0.04 \\
0.04\end{array}$ & $\begin{array}{r}<0.02 \\
<0.02 \\
0.04 \\
0.04\end{array}$ & --- & \\
\hline & $\begin{array}{l}\text { Plant Drinking Water } \\
\text { OW-WR-23 } \\
\text { OW-WR-28 }\end{array}$ & $\begin{array}{l}2 \\
2\end{array}$ & $\begin{array}{l}<0.02 \\
<0.03\end{array}$ & $\begin{array}{r}<0.02 \\
0.04\end{array}$ & $\begin{array}{l}<0.02 \\
<0.02\end{array}$ & $-\cdots$ & \\
\hline & $\begin{array}{c}\text { Monitor Wells } \\
\text { OW-WR-19 } \\
\text { OW-WR-20 } \\
\text { OW-WR-38 } \\
\text { OW-WR-39 } \\
\text { OW-WR-40 }\end{array}$ & $\begin{array}{l}2 \\
2 \\
2 \\
1 \\
1\end{array}$ & $\begin{array}{r}<0.02 \\
<0.02 \\
0.09 \\
0.03 \\
<0.02\end{array}$ & $\begin{array}{r}0.03 \\
0.02 \\
0.12 \\
0.03 \\
<0.02\end{array}$ & $\begin{array}{r}<0.02 \\
<0.02 \\
0.07 \\
0.03 \\
<0.02\end{array}$ & --- & \\
\hline & $\begin{array}{l}\text { Industrial Waters } \\
\text { OW-WR-09 } \\
\text { OW-WR-13 } \\
\text { OW-WR-15 } \\
\text { OW-WR-17 } \\
\text { OW-WR-25 } \\
\text { OW-WR-31 }\end{array}$ & $\begin{array}{l}8 \\
8 \\
6 \\
8 \\
7 \\
8\end{array}$ & $\begin{array}{l}<0.07 \\
<0.11 \\
<0.02 \\
1.04 \\
<0.02 \\
<0.02\end{array}$ & $\begin{array}{l}0.17 \\
0.66 \\
0.02 \\
1.30 \\
0.04 \\
0.03\end{array}$ & $\begin{array}{r}<0.02 \\
<0.02 \\
<0.02 \\
0.65 \\
<0.02 \\
<0.02\end{array}$ & --- & \\
\hline & $\begin{array}{l}\text { Control } \\
\text { OW-WR-33 } \\
\text { OW-WR-37 }\end{array}$ & $\begin{array}{l}9 \\
8\end{array}$ & $\begin{array}{l}<0.02 \\
<0.02\end{array}$ & $\begin{array}{l}0.03 \\
0.03\end{array}$ & $\begin{array}{l}<0.02 \\
<0.05\end{array}$ & $\cdots$ & \\
\hline
\end{tabular}


Table XXXIV. Surmary of Chemical Analysis for Water Pollutants for 1988 (Cont 'd)

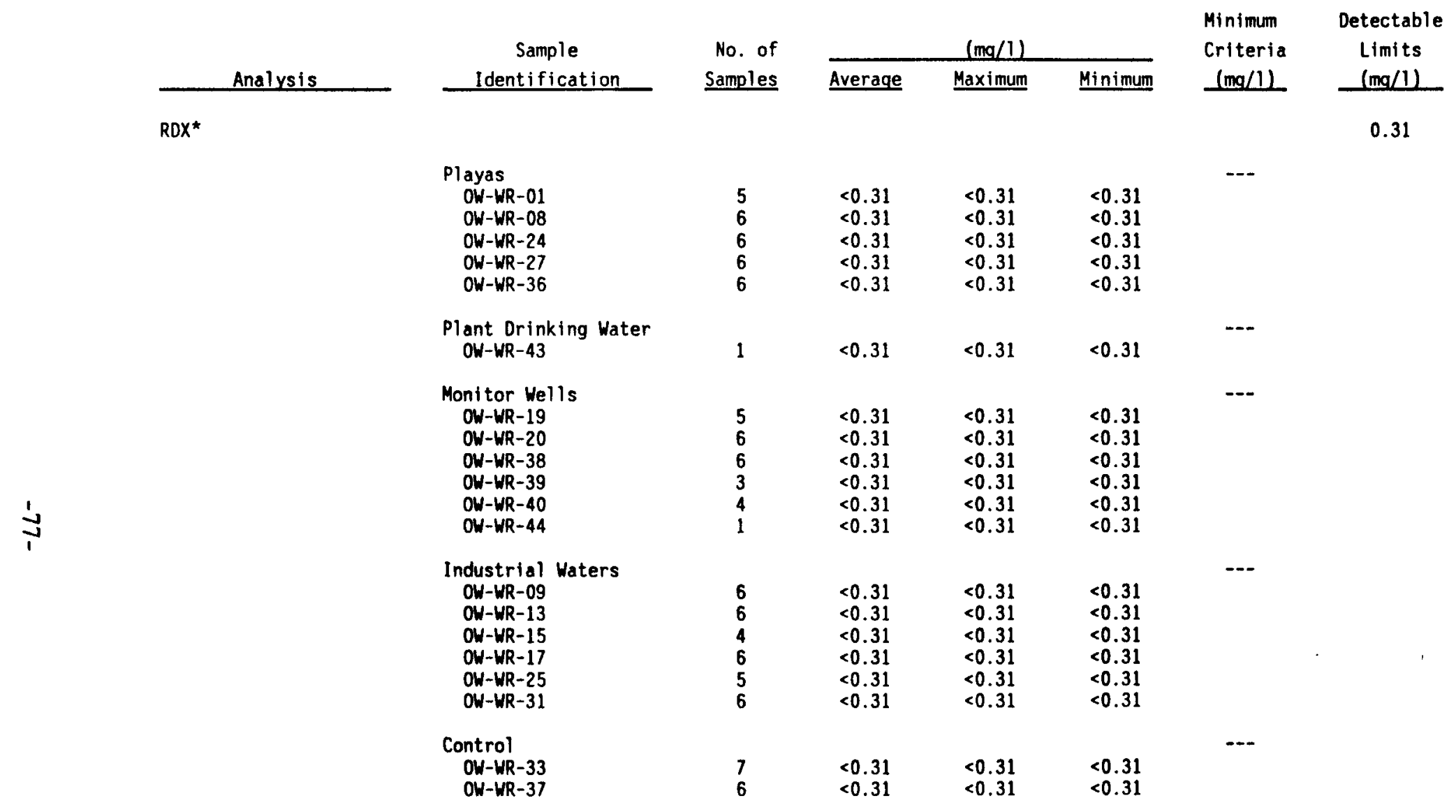

RDX, HMX, and PETN are crystalline high explosives used at Pantex Plant. 
Table XXXIV. Summary of Chemical Analysis for Water Pollutants for 1988 (Cont'd)

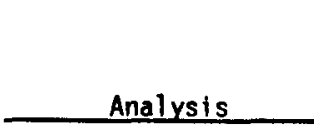

Selenium (Se)
Sample

Identification

Playas

OW-WR-01
OW-WR-08

OW-WR-24

Water Wells

OW-WR-02

OW-WR-06

OW-WR-16

OW-WR-18

Plant Drinking Water

OW-WR-23

OW-WR-43

Monitor Wells

OW-WR-19

OW-WR-20

OW-WR-38

OW-WR-39

OW-WR-40

Industrial Waters

OW-WR-09

OW-WR-13

OW-WR-15

OW-WR-17

OW-WR-25

OW-WR-31

Control

OW-WR-33

OW-WR-37
No. of

Samples

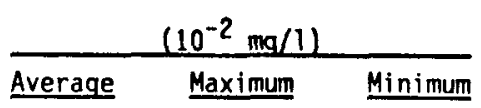

Maximum

Minimum

1
2
2

3
3
4
4

$<0.5$
$<0.5$
$<0.5$

$<0.5$

$<0.5$

$<0.5$

$<0.5$

$\begin{array}{lll}<0.5 & <0.5 & <0.5 \\ <0.5 & <0.5 & <0.5 \\ <0.5 & <0.5 & <0.5 \\ <0.5 & <0.5 & <0.5\end{array}$

$4<0.5$

$<0.5$
$<0.5$

$<0.5$
$<0.5$

$<0.5$

$<0.5$

$<0.5$

$<0.5$

$<0.5$

$2<0$

$<0.5$

$<0.5$

$<0.5$
$<0.5$

$<0.5$
$<0.5$

$<0.5$

$<0.5<0.5$

$\begin{array}{ll}2 & <0.5 \\ 2 & <0.5 \\ 2 & <0.5 \\ 2 & <0.5 \\ 2 & <0.5 \\ 2 & <0.5\end{array}$

$<0.5$

$<0.5$

$<0.5$

$<0.5$

$<0.5$

$<0.5<0.5$

$<0.5<0.5$

$<0.5<0.5$

$<0.5<0.5$

$<0.5<0.5$

$\begin{array}{ll}9 & <0.5 \\ 8 & <0.5\end{array}$

$<0.5$

$<0.6$

$<0.5$

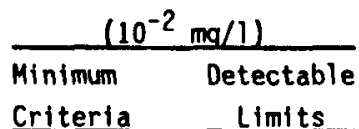

0.5

5.0

1.0

1.0

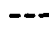

$\cdots$

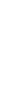


Table XXXIV. Summary of Chemical Analysis for Water Pollutants for 1988 (Cont'd)

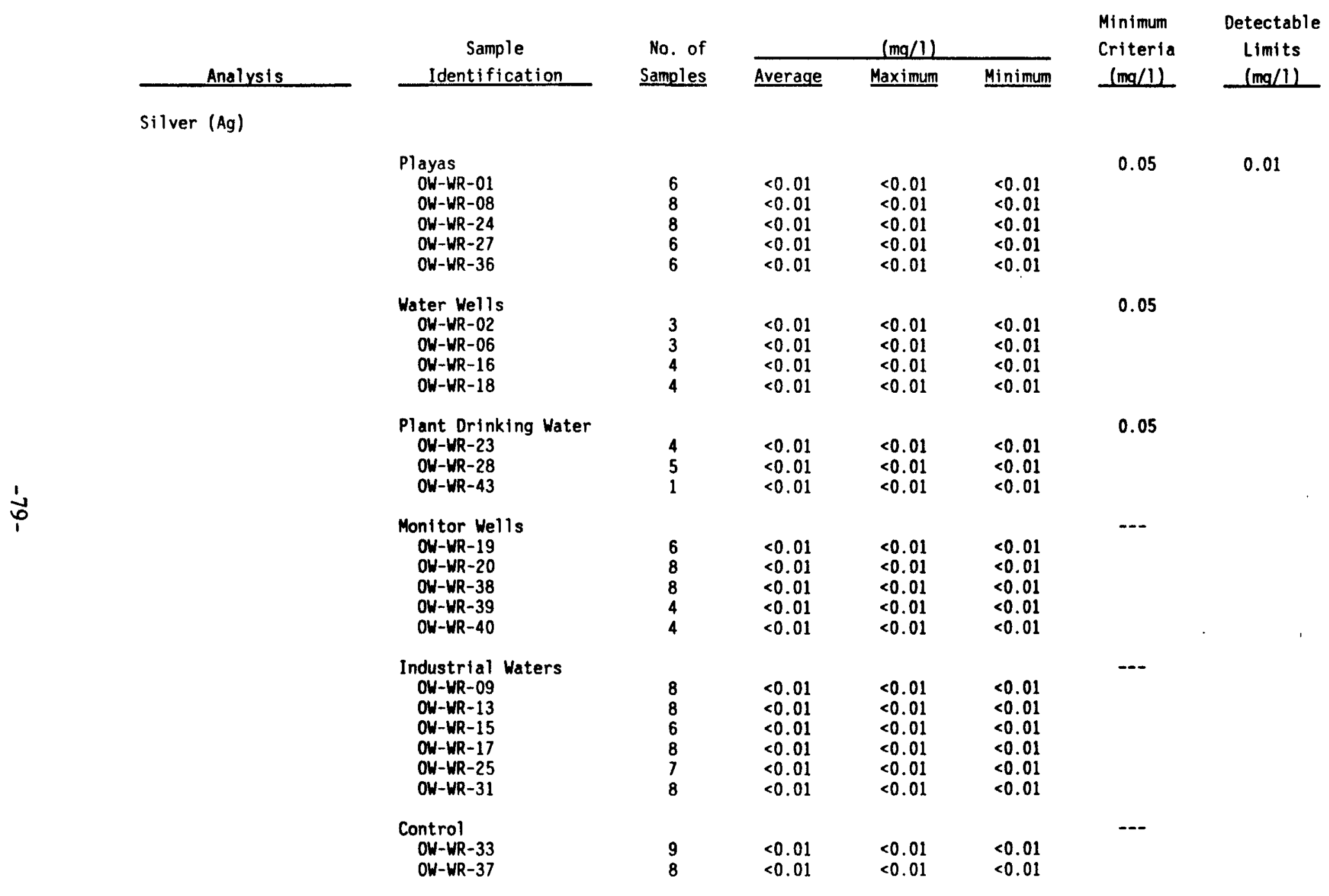


Table XXXIV. Summary of Chemical Analysis for Water Pollutants for 1988 (Cont'd)

\begin{tabular}{|c|c|c|c|c|c|c|c|}
\hline \multirow[b]{2}{*}{ Analysis } & \multirow{2}{*}{$\begin{array}{c}\text { Sample } \\
\text { Identification }\end{array}$} & \multirow{2}{*}{$\begin{array}{l}\text { No. of } \\
\text { Samples }\end{array}$} & \multicolumn{3}{|c|}{$(\mathrm{mg} / 1)$} & \multirow{2}{*}{$\begin{array}{l}\text { Minimum } \\
\text { Criteria } \\
\text { (ma/1) }\end{array}$} & \multirow{2}{*}{$\begin{array}{c}\text { Detectable } \\
\text { Limits } \\
\text { (mg/1) } \\
\end{array}$} \\
\hline & & & Average & Maximum & Minimum & & \\
\hline Sulfate $\left(\mathrm{SO}_{4}\right)^{-2^{\star}}$ & $\begin{array}{c}\text { Monitor Wells } \\
\text { OW-WR-19 } \\
\text { OW-WR-20 } \\
\text { OW-WR-38 } \\
\text { OW-WR-40 }\end{array}$ & $\begin{array}{l}4 \\
6 \\
6 \\
3\end{array}$ & $\begin{array}{l}13.7 \\
34.0 \\
71.0 \\
16.0\end{array}$ & $\begin{array}{l}40.0 \\
40.0 \\
98.0 \\
23.0\end{array}$ & $\begin{array}{r}<5.0 \\
30.0 \\
22.0 \\
<05.0\end{array}$ & $\cdots$ & 5.0 \\
\hline & $\begin{array}{l}\text { Industrial Waters } \\
\text { OW-WR-09 } \\
\text { OW-WR-13 } \\
\text { OW-WR-15 } \\
\text { OW-WR-17 } \\
\text { OW-WR-25 } \\
\text { OW-WR-31 }\end{array}$ & $\begin{array}{l}6 \\
5 \\
4 \\
6 \\
5 \\
6\end{array}$ & $\begin{array}{r}32.1 \\
48.4 \\
27.5 \\
109.5 \\
26.2 \\
28.5\end{array}$ & $\begin{array}{r}43.0 \\
120.0 \\
32.0 \\
150.0 \\
33.0 \\
32.0\end{array}$ & $\begin{array}{l}23.0 \\
24.0 \\
24.0 \\
35.0 \\
13.0 \\
25.0\end{array}$ &.-- & \\
\hline & $\begin{array}{l}\text { Control } \\
\text { OW-WR-33 } \\
\text { OW-WR-37 }\end{array}$ & $\begin{array}{l}7 \\
6\end{array}$ & $\begin{array}{l}41.4 \\
28.5\end{array}$ & $\begin{array}{l}46.0 \\
33.0\end{array}$ & $\begin{array}{l}34.0 \\
23.0\end{array}$ & $\cdots$ & \\
\hline
\end{tabular}

*Samples only taken where sulfate from plant operations may infiltrate. 
Table XXXIV. Sumary of Chemical Analysis for Water Pollutants for 1988 (Cont ${ }^{\circ} d$ )

\begin{tabular}{|c|c|c|c|c|c|c|c|c|}
\hline & \multirow[b]{3}{*}{ Analysis } & \multirow{3}{*}{$\begin{array}{c}\text { Sample } \\
\text { Identification }\end{array}$} & \multirow{3}{*}{$\begin{array}{l}\text { No. of } \\
\text { Samples } \\
\end{array}$} & \multirow{2}{*}{\multicolumn{3}{|c|}{$\left(10^{-3} \mathrm{mg} / 1\right)$}} & \multicolumn{2}{|c|}{$\left(10^{-3} \mathrm{mg} / 1\right)$} \\
\hline & & & & & & & \multirow{2}{*}{$\begin{array}{l}\text { Minimum } \\
\text { Criteria }\end{array}$} & \multirow{2}{*}{$\begin{array}{l}\text { Detectable } \\
\text { Limits } \\
\end{array}$} \\
\hline & & & & Average & Maximum & Minimum & & \\
\hline & Tetrahydrofuran & & & & & & & 5.0 \\
\hline & & $\begin{array}{c}\text { Water We11s } \\
\text { OW-WR-02 } \\
\text { OW-WR-06 } \\
\text { OW-WR-16 } \\
\text { OW-WR-18 }\end{array}$ & $\begin{array}{l}1 \\
1 \\
1 \\
1\end{array}$ & $\begin{array}{l}<5.0 \\
<5.0 \\
<5.0 \\
<5.0\end{array}$ & $\begin{array}{l}<5.0 \\
<5.0 \\
<5.0 \\
<5.0\end{array}$ & $\begin{array}{l}<5.0 \\
<5.0 \\
<5.0 \\
<5.0\end{array}$ & -- & \\
\hline & & $\begin{array}{l}\text { Plant Drinking Water } \\
\text { OW-WR-43 }\end{array}$ & 1 & $<5.0$ & $<5.0$ & $<5.0$ & -- & \\
\hline & & $\begin{array}{c}\text { Monitor Wells } \\
\text { OW-WR-19 } \\
\text { OW-WR-20 } \\
\text { OW-WR-38 } \\
\text { OW-WR-39 } \\
\text { OW-WR-40 } \\
\text { OW-WR-44 }\end{array}$ & $\begin{array}{l}\cdot 1 \\
1 \\
1 \\
1 \\
1 \\
1\end{array}$ & $\begin{array}{l}<5.0 \\
<5.0 \\
<5.0 \\
<5.0 \\
<5.0 \\
<5.0\end{array}$ & $\begin{array}{l}<5.0 \\
<5.0 \\
<5.0 \\
<5.0 \\
<5.0 \\
<5.0\end{array}$ & $\begin{array}{l}<5.0 \\
<5.0 \\
<5.0 \\
<5.0 \\
<5.0 \\
<5.0\end{array}$ & -- & \\
\hline 1 & & $\begin{array}{l}\text { Control } \\
\text { OW-WR-37 }\end{array}$ & 1 & $<5.0$ & $<5.0$ & $<5.0$ & --- & \\
\hline
\end{tabular}


Table XxxIV. Surmary of Chemical Analysis for Water Pollutants for 1988 (Cont'd)

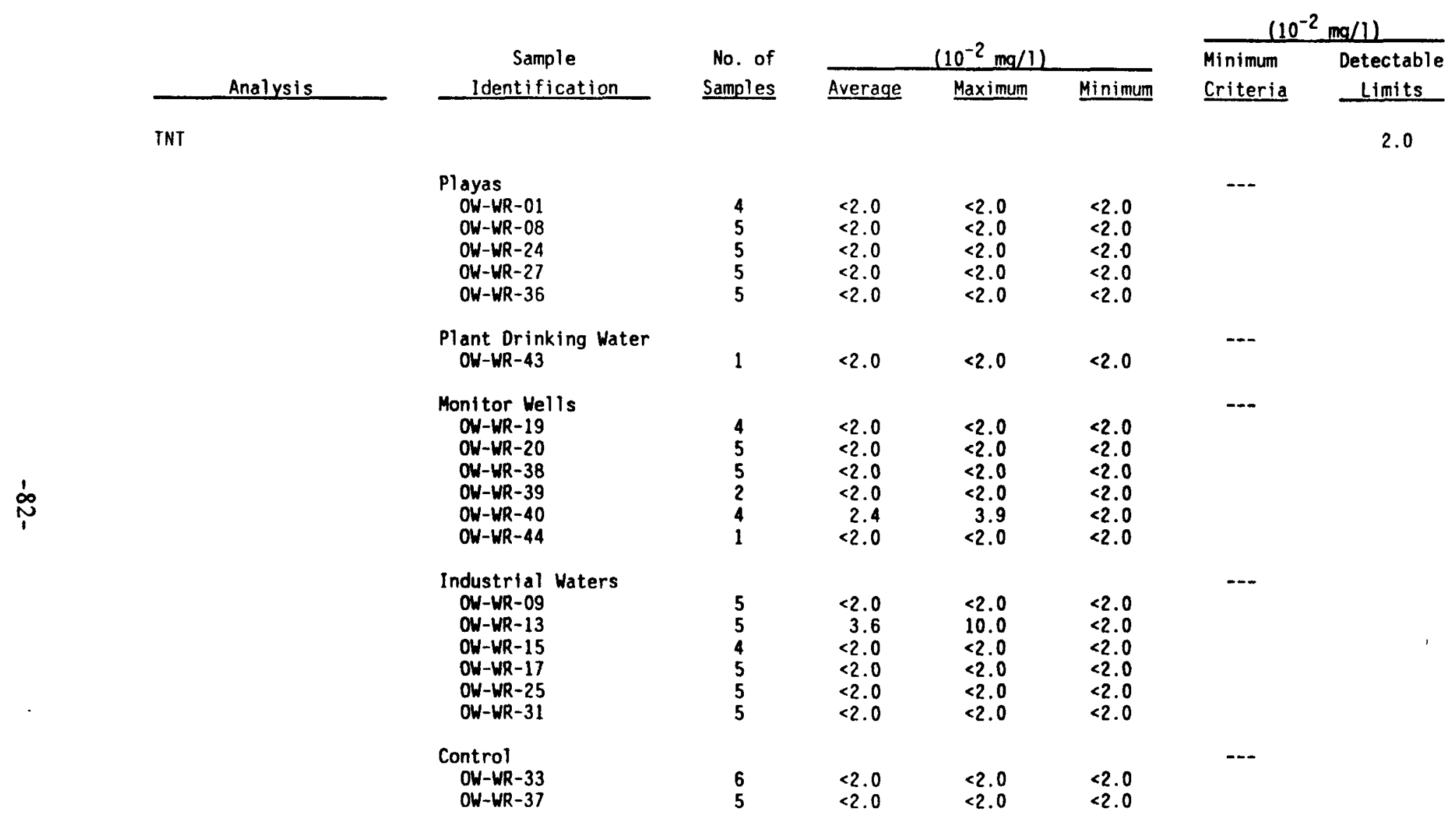


Table XXXIV. Summary of Chemical Analysis for Water Pollutants for 1988 (Cont'd)

\begin{tabular}{|c|c|c|c|c|c|c|c|}
\hline \multirow[b]{3}{*}{ Analysis } & \multirow{3}{*}{$\begin{array}{c}\text { Sample } \\
\text { Identification } \\
\end{array}$} & \multirow{3}{*}{$\begin{array}{l}\text { No. of } \\
\text { Samples }\end{array}$} & \multirow{2}{*}{\multicolumn{3}{|c|}{$\left(10^{-3} \mathrm{mg} / 1\right)$}} & \multicolumn{2}{|c|}{$\left(10^{-3} \mathrm{mg} / 1\right)$} \\
\hline & & & & & & \multirow{2}{*}{$\begin{array}{l}\text { Minimum } \\
\text { Criteria }\end{array}$} & \multirow{2}{*}{$\begin{array}{l}\text { Detectable } \\
\text { Limits } \\
\end{array}$} \\
\hline & & & Average & Maximum & Minimum & & \\
\hline \multirow[t]{16}{*}{ Toluene } & & & & & & & 5.0 \\
\hline & Water Wells & & & & & --- & \\
\hline & OW-WR-02 & 1 & $<5.0$ & $<5.0$ & $<5.0$ & & \\
\hline & OW-WR-06 & 1 & $<5.0$ & $<5.0$ & $<5.0$ & & \\
\hline & $\begin{array}{l}\text { OW-WR-16 } \\
\text { OW-WR-18 }\end{array}$ & 1 & $<5.0$ & $<5.0$ & $<5.0$ & & \\
\hline & & & & & & & \\
\hline & Plant Drinking Water & & & & & -- & \\
\hline & OW-WR-43 & 1 & $<5.0$ & $<5.0$ & $<5.0$ & & \\
\hline & Monitor Wells & & & & & --- & \\
\hline & OW-WR-19 & 1 & $<5.0$ & $<5.0$ & $<5.0$ & & \\
\hline & $O W-W R-20$ & $i$ & $<5.0$ & $<5.0$ & $<5.0$ & & \\
\hline & OW-WR-38 & 1 & $<5.0$ & $<5.0$ & $<5.0$ & & \\
\hline & OH-WR-39 & 1 & $<5.0$ & $<5.0$ & $<5.0$ & & \\
\hline & $\begin{array}{l}O W-W R-40 \\
\text { OW-WR-44 }\end{array}$ & 1 & $<5.0$ & $<5.0$ & $<5.0$ & & \\
\hline & Control & & & & & -- & \\
\hline & OW-WR-37 & 1 & $<5.0$ & $<5.0$ & $<5.0$ & & \\
\hline
\end{tabular}


Table XXXIV. Surmary of Chemical Analysis for Water Pollutants for 1988 (Cont 'd)

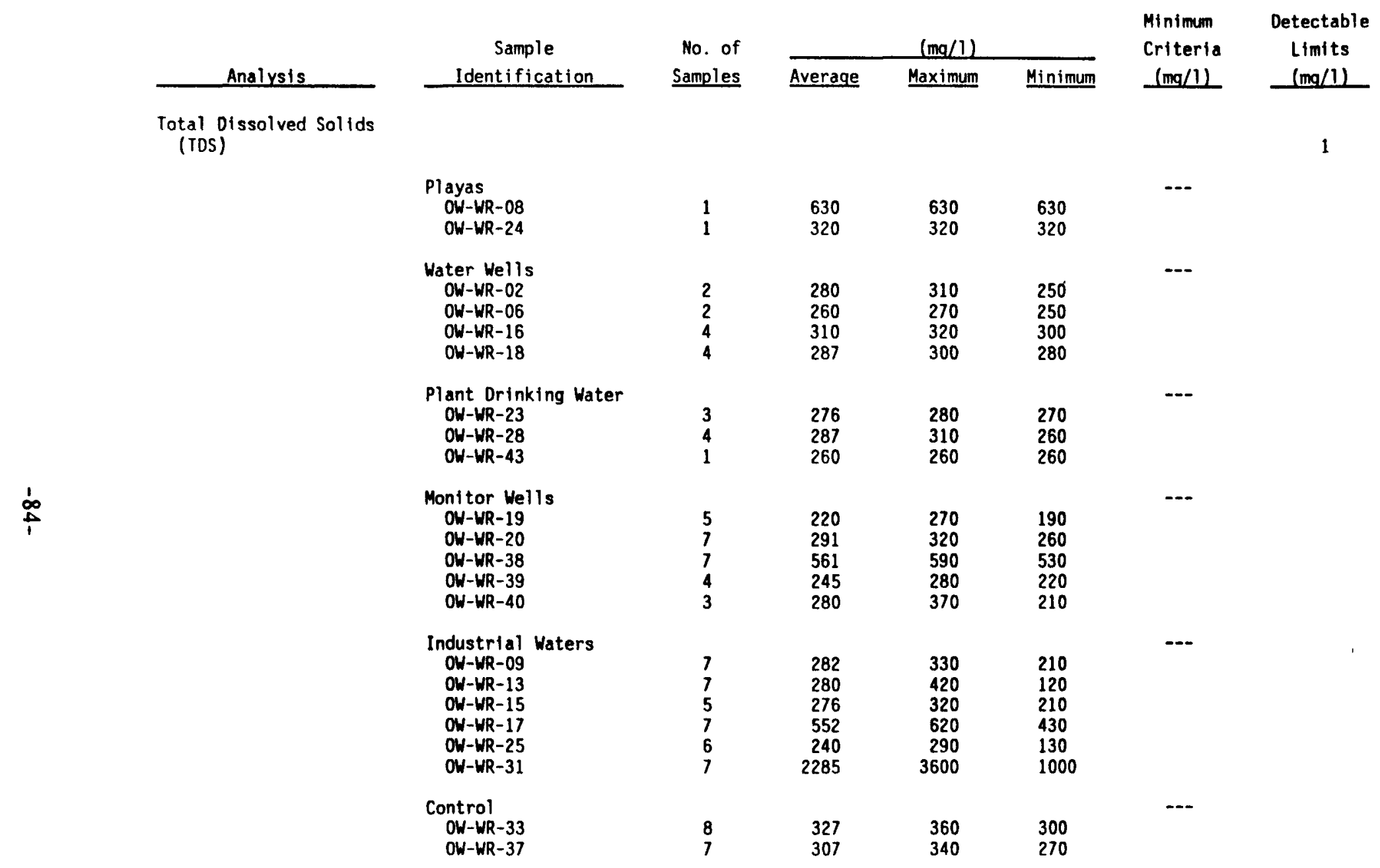


Table XXXIV. Summary of Chemical Analysis for Water Pollutants for 1988 (Cont'd)

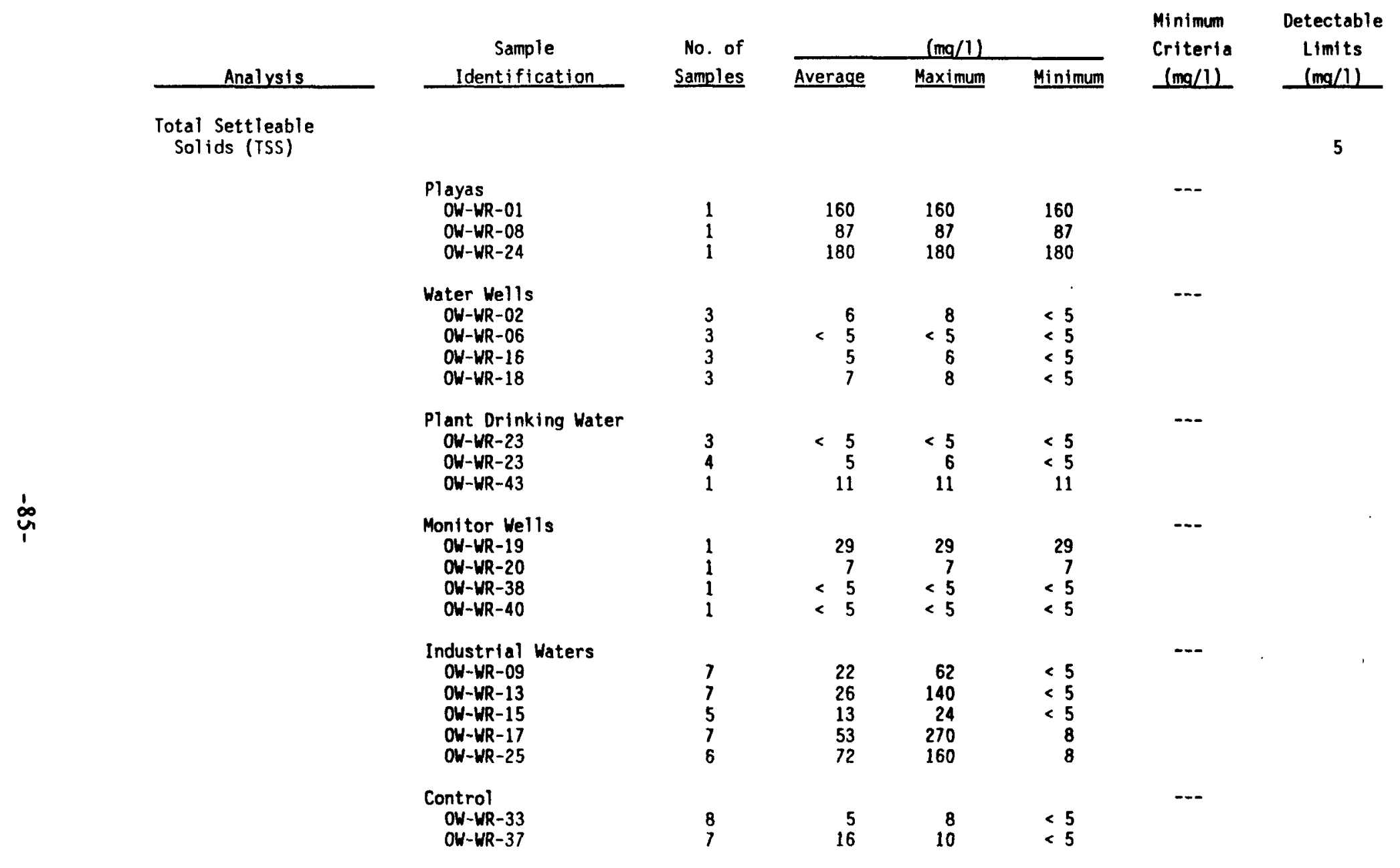


Table XXXIV. Summary of Chemical Analysis for Water Pollutants for 1988 (Cont' ${ }^{\circ}$ )

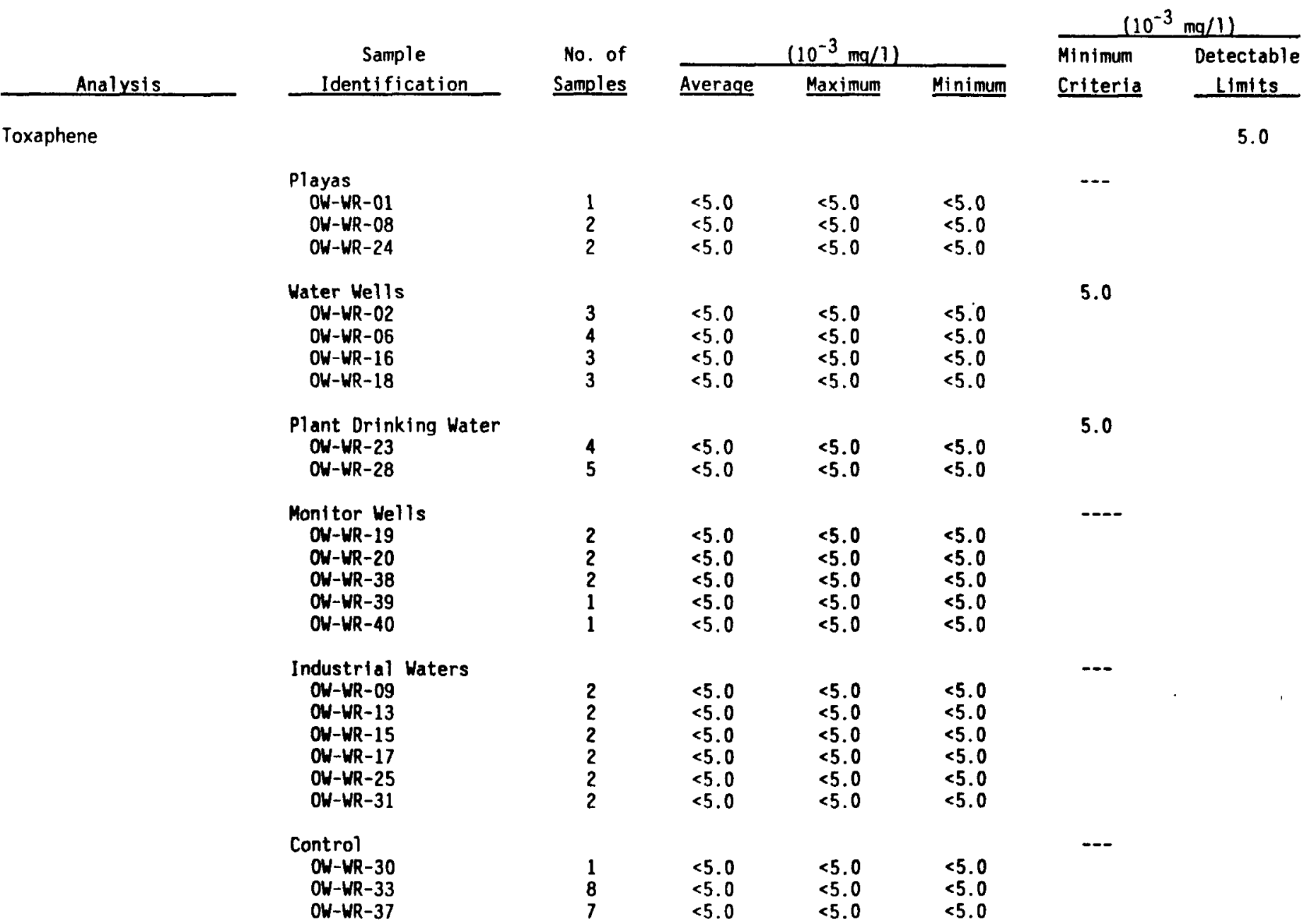


Table XXXIV. Summary of Chemical Analysis for Water Pollutants for 1988 (Cont'd)

\begin{tabular}{|c|c|c|c|c|c|c|c|c|}
\hline & \multirow[b]{3}{*}{ Analysis } & \multirow{3}{*}{$\begin{array}{c}\text { Sample } \\
\text { Identification }\end{array}$} & \multirow{3}{*}{$\begin{array}{r}\text { No. of } \\
\text { Samples } \\
\end{array}$} & \multirow{2}{*}{\multicolumn{3}{|c|}{$\left(10^{-3} \mathrm{mg} / 1\right)$}} & \multicolumn{2}{|c|}{$\left(10^{-3} \mathrm{mg} / 1\right)$} \\
\hline & & & & & & & \multirow{2}{*}{$\begin{array}{l}\text { Minimum } \\
\text { Criteria }\end{array}$} & \multirow{2}{*}{$\begin{array}{l}\text { Detectable } \\
\text { Limits } \\
\end{array}$} \\
\hline & & & & Average & Maximum & Minimum & & \\
\hline & Total Xylenes & & & & & & & 5.0 \\
\hline & & $\begin{array}{c}\text { Water Wel1s } \\
\text { OW-WR-02 } \\
\text { OW-WR-06 } \\
\text { OW-WR-16 } \\
\text { OW-WR-18 }\end{array}$ & $\begin{array}{l}1 \\
1 \\
1 \\
1\end{array}$ & $\begin{array}{l}<5.0 \\
<5.0 \\
<5.0 \\
<5.0\end{array}$ & $\begin{array}{l}<5.0 \\
<5.0 \\
<5.0 \\
<5.0\end{array}$ & $\begin{array}{l}<5.0 \\
<5.0 \\
<5.0 \\
<5.0\end{array}$ &.-- & \\
\hline & & $\begin{array}{l}\text { Plant Drinking Water } \\
\text { OW-WR-43 }\end{array}$ & . 1 & $<5.0$ & $<5.0$ & $<5.0$ & -- & \\
\hline & & $\begin{array}{c}\text { Monitor Wells } \\
\text { OW-WR-19 } \\
\text { OW-WR-20 } \\
\text { OW-WR-38 } \\
\text { OW-WR-39 } \\
\text { OW-WR-40 } \\
\text { OW-WR-44 }\end{array}$ & $\begin{array}{l}1 \\
1 \\
1 \\
1 \\
1 \\
1\end{array}$ & $\begin{array}{l}<5.0 \\
<5.0 \\
<5.0 \\
<5.0 \\
<5.0 \\
<5.0\end{array}$ & $\begin{array}{l}<5.0 \\
<5.0 \\
<5.0 \\
<5.0 \\
<5.0 \\
<5.0\end{array}$ & $\begin{array}{l}<5.0 \\
<5.0 \\
<5.0 \\
<5.0 \\
<5.0 \\
<5.0\end{array}$ & -.- & \\
\hline
\end{tabular}


Table XXXIV. Summary of Chemical Analysis for Water Pollutants for 1988 (Cont'd)

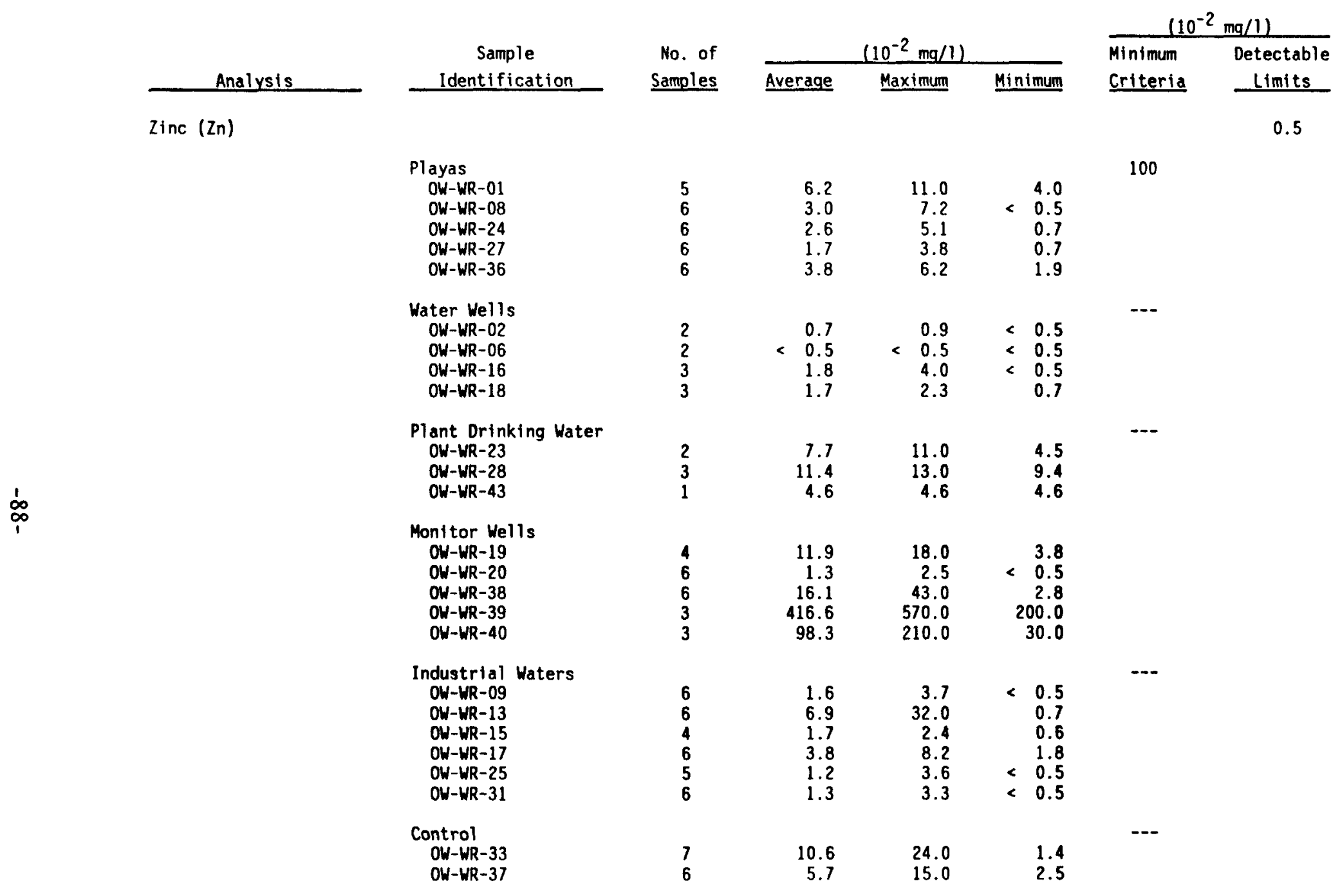


Table XXXIV. Summary of Chemical Analysis for Water Pollutants for 1988 (Cont 'd)

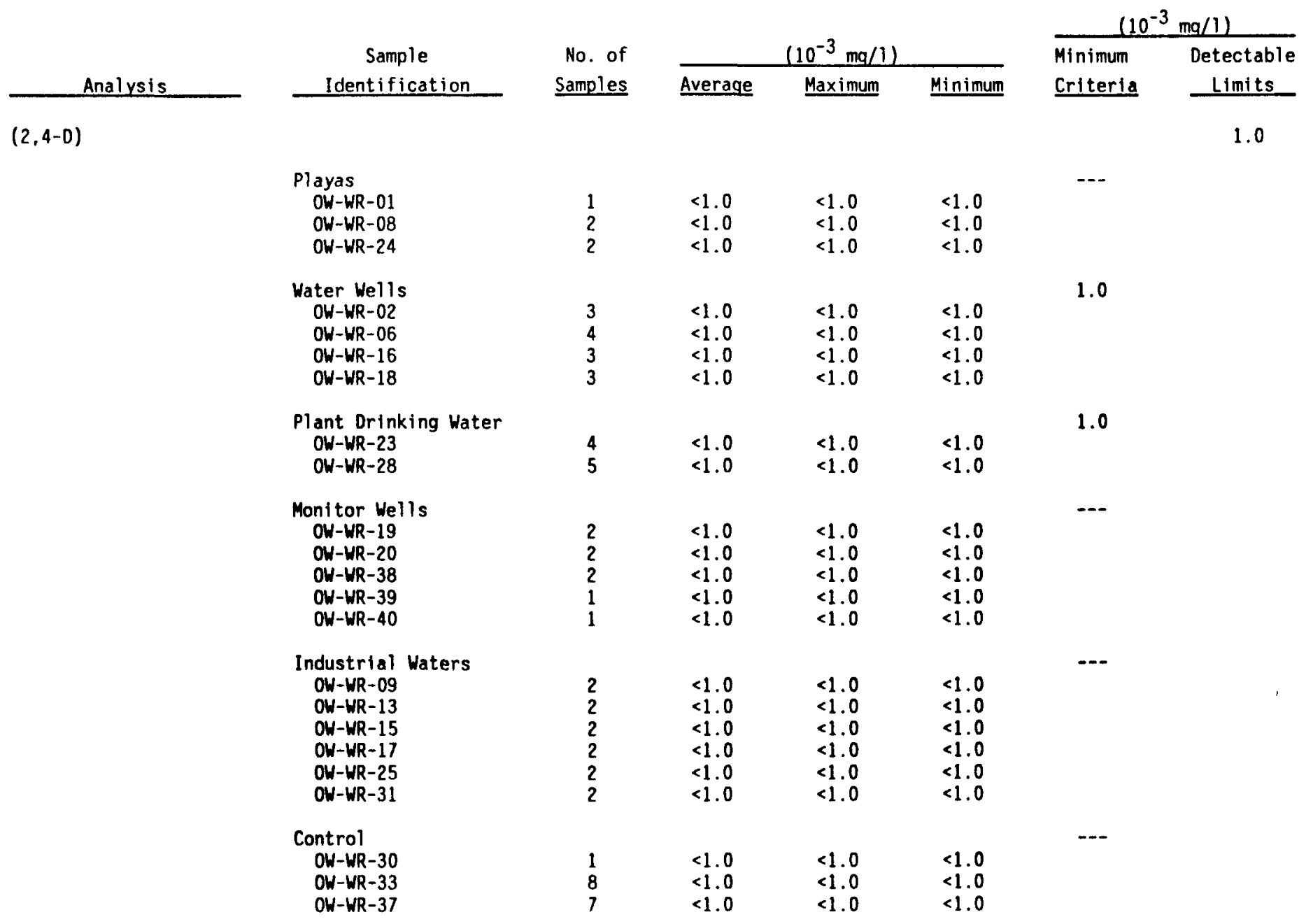


Table XXXIV. Surmary of Chemical Analysis for Water Pollutants for 1988 (Cont'd)

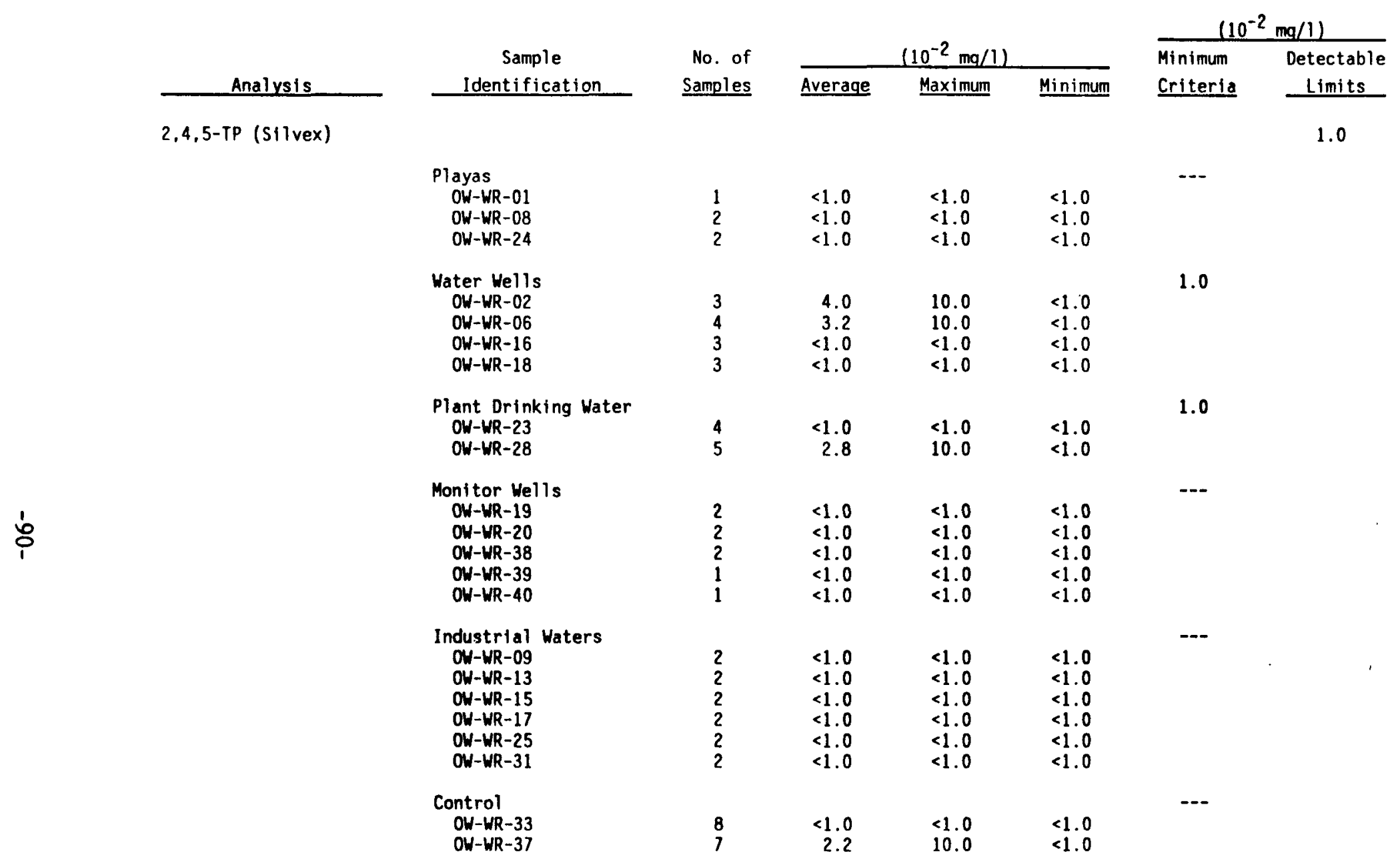


Table XxxIV. Summary of Chemical Analysis for Water Pollutants for 1988 (Cont'd)

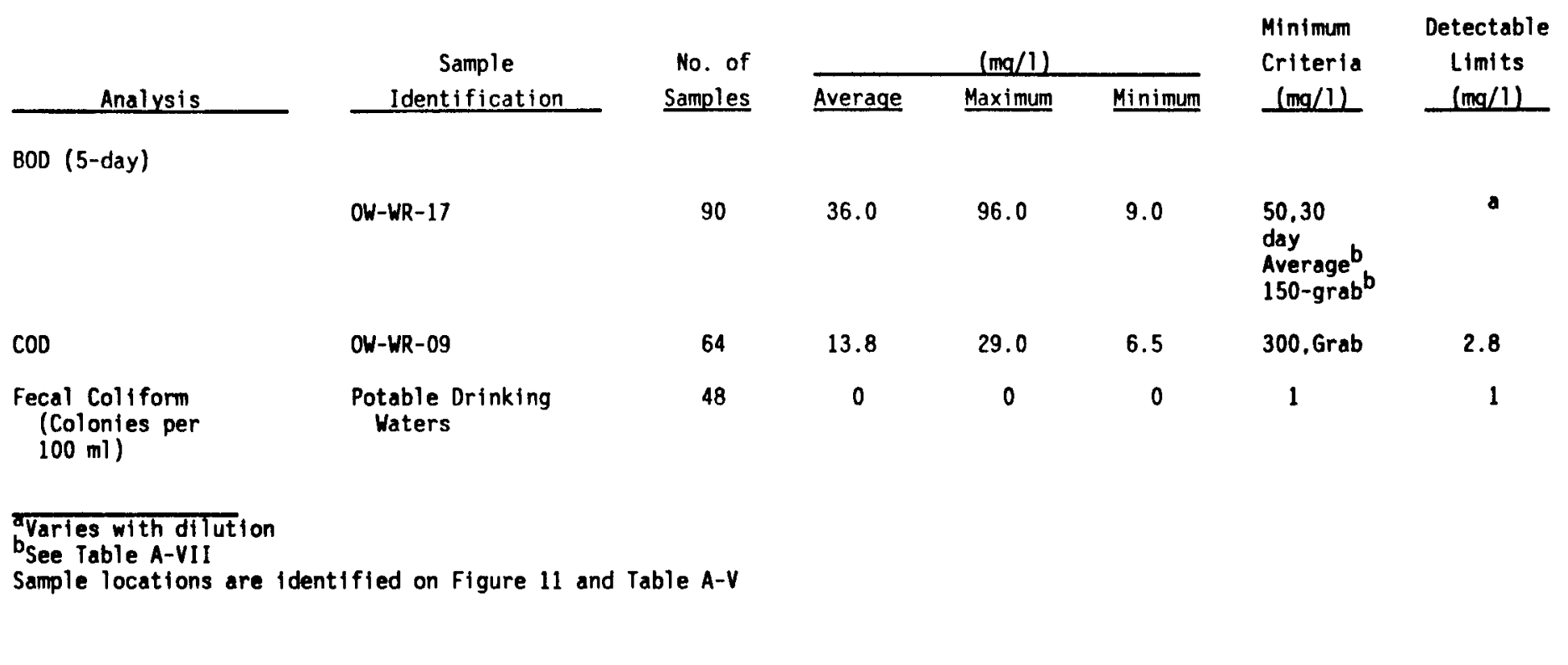




\section{REFERENCES}

1. U.S. Department of Energy, DOE Explosives Safety Manual, DOE/EV/ 06194-3 Rev. 2 (August 1985).

2. U.S. Army, DARCOM Regulation Safety Manual, DARCOM 385-100 (August 1985).

3. Letter Cecil L. Bradford, Texas Air Control Board to P. R. Wagner, United States Energy Research and Development Administration (dated July 9, 1976).

4. Energy and Air Ouality, U.S. Department of Energy, Report Number DOE/EV/10154-3 (December 1981).

5. McDonald, James R., "Assessment of Tornado and Straight Wind Risks at the Pantex, Texas Site," McDonald, Menta and Minor Consulting Engineers (December 1979).

6. Seismic Hazard Analysis. Pantex Ordnance Plant. Amarillo. Texas, TERA Corporation, Berkeley, CA (May 1980) (Draft).

7. Uniform Building Code (1988).

8. Percolation of Water Through Pullman Soils. Texas High Plains, The Texas Agricultural Experimental Station (1971).

9. Basin Recharge of the Ogallala Aouifer, Southwestern Great Plains Research Center (1972).

10. Hydrogeology of a Playa Near Amarillo, Texas, Southwestern Great Plains Research Center (1971).

11. Final Environmental Impact Statement. Pantex Plant Site. Amarillo. Texas. U.S. Department of Energy, Report Number DOE/EIS-0098 (October 1983).

12. 1980 Census of Population and Housing, U.S. Department of Commerce, Report No. PHC80-V-45 (March 1981).

13. Local Climatological Data, U.S. Department of Commerce, National Oceanic and Atmospheric Administration (January 1986 through December 1986).

14. User's Guide for the Climatological Dispersion Model, EPA-R4-73-024, U.S. Environmental Protection Agency (December 1973).

15. DOE Order 5480.1, Chapter XI, Requirements for Radiation Protection.

16. William A. Vaughan, Radiation Standards for Protection of the Public in the Vicinity of DOE Facilities (August 5, 1985).

17. National Interim Primary Drinking Water Regulations, Title 40, Part 141, Environmental Protection Agency (July 1, 1987). 
18. "Drinking Water Standards Governing Drinking Water Quality and Reporting Requirements for Public Water Supply Systems," 25 TAC 337, Texas Department of Health, Austin, Texas.

19. Standard Methods for the Examination of Water and Waste Water, Fifteenth Edition, American Public Health Association, New York, NY (1980).

20. Methods for Chemical Analyses of Water and Wastes, Environmental Protection Agency, Water Quality Office, Analytical Control Laboratory, Cincinnati, Ohio, 1971, 16020 (July 1971).

21. Analysis of Pesticide Residues in Human and Environmental Samples, Environmental Protection Agency, Toxicology Division, Research Triangle Park, NC, 27711 (June 1977).

22. Draft DOE Order 5480.XX, Radiation Protection of the Public and the Environment.

23. G. W. Dawson, "The Chemical Toxicity of Elements," Pacific Northwest Laboratories, Report Number BNWL-1815 (June 1974). 


\section{APPENDIX A}

\section{STANDARDS FOR RADIOACTIVITY IN AIR AND WATER}

Standards for radioactivity in air and water are derived from DOE Order 5480.1A (15) for controlled (plant-wide) areas and from the memo "Radiation Standards for Protection of the Public in the Vicinity of DOE Facilities," dated August 5, 1985, by William A. Vaughan, Assistant Secretary of Environment, Safety and Health Division of $\operatorname{DOE}(16)$, for off site (uncontrolled or general population) areas.

Controlled area exposures are set to limit dose equivalents to levels presented in Table A-I.

Table A-I. Controlled Area Exposures

\begin{tabular}{|c|c|c|}
\hline Type of Exposure & Exposure Period & $\begin{array}{l}\text { Dose Equivalent } \\
\text { (Dose or Dose } \\
\text { Cormitment, ren) }\end{array}$ \\
\hline $\begin{array}{l}\text { Whole body, head and trunk, } \\
\text { gonads, lens of the eye, red } \\
\text { bone marrow, active blood- } \\
\text { forming organs }\end{array}$ & $\begin{array}{c}\text { Year } \\
\text { Calendar Quarter }\end{array}$ & $\begin{array}{l}5 \\
3\end{array}$ \\
\hline $\begin{array}{l}\text { Unlimited areas of the skin } \\
\text { (except hands and forearms) } \\
\text { Other organs, tissues, and } \\
\text { organ systems (except bone) }\end{array}$ & $\begin{array}{c}\text { Year } \\
\text { Calendar Quarter }\end{array}$ & $\begin{array}{r}15 \\
5\end{array}$ \\
\hline Bone & $\begin{array}{c}\text { Year } \\
\text { Calendar Quarter }\end{array}$ & $\begin{array}{l}30 \\
10\end{array}$ \\
\hline Forearms & $\begin{array}{c}\text { Year } \\
\text { Calendar Quarter }\end{array}$ & $\begin{array}{l}30 \\
10\end{array}$ \\
\hline Hands and feet & $\begin{array}{c}\text { Year } \\
\text { Calendar Quarter }\end{array}$ & $\begin{array}{l}75 \\
25\end{array}$ \\
\hline
\end{tabular}

Off site exposure levels are set to limit effective dose equivalents from all pathways due to routine plant operation to 100 mrem per year.

This exposure is considered continuous. The air pathway is further restricted to 25 mrem/year whole body dose or $75 \mathrm{mrem} /$ year to any organ, by $40 \mathrm{CFR} 61$ Subpart H, "National Emission Standard for Radionuclide Emissions" from Department of Energy (DOE) Facilities. 


\section{RADIOACTIVITY CONCENTRATION GUIDES FOR SOIL AND VEGETATION}

Presently there are no concentration guides for radionuclides in soil and vegetation.

\section{NONRADIOACTIVE WATER QUALITY STANDARDS}

Nonradioactive analyses are compared to applicable Environmental Protection Agency (EPA) Primary Drinking Water Standards 40 CFR 141, and Texas Department of Health Regulations, 25 TAC 337(17,18).

\section{STATISTICAL METHODS}

Nonradioactive measurements are based on analog representations of physical parameters and a lower limit exists as to the sensitivity of the techniques and instrumentation employed. When measurements of nonradioactive parameters were performed and no indication in excess of lower detection limit was observed, the result is presented as less than (<) the minimum detectable limit. Average values were calculated by assuming the minimum detectable limit where positive indications were not obtained and portrayals of the final average as a less than value. The less than value for an average therefore indicates the assumption of the minimum detectable limit for one or more terms within the average.

Radioactive measurements, however, are subject to the statistical nature of radioactive decay and are presented in conjunction with an associated statistical error. All errors presented represent counting errors only; no attempt has been made to quantify systematic errors. The minimum detectable limits of the radioactive parameters were calculated based on USNRC Regulatory Guide 4.14. Negative numbers can arise from statistical fluctuations in the radioactive decay of the sample, the blank, or in the background of the counting device. Averages which are less than zero in value (i.e., negative) are presented as being less than the minimum detectable limit.

\section{ANALYTICAL METHODS (NONRADIOACTIVE)}

\section{WATER}

In general, nonradioactive analyses of water were performed in accordance with standard methods(19,20). High explosives were analyzed by High Performance Liquid Chromatography (HPLC) utilizing a UV absorption detector. Pesticides were analyzed by vapor phase gas chromatography(21). 


\title{
ANALYTICAL METHODS (RADIOACTIVE)
}

\begin{abstract}
AIR
AIR FILTERS

Gross Alpha, Gross Beta

Filter paper samples are counted for Gross Alpha and Gross Beta radioactivity on a low background internal flow proportional counter which simultaneously counts alpha and beta activity. Appropriate corrections are applied for absorption and counter efficiency.
\end{abstract}

\section{Uranium-234 and -238}

The sample is digested to dryness with strong acids and redissolved in $5 \% \mathrm{HNO}_{3}$. The uranium is separated by co-precipitation with $\mathrm{AlPO}_{4}$. Then the precipitate is dissolved in $2 \mathrm{~N} \mathrm{Al}\left(\mathrm{NO}_{3}\right)_{3}$ and extracted into ethyl acetate. The ethyl acetate is dried and the sample transferred to a stainless steel planchet for counting in an internal flow proportional counter for its alpha activity.

\section{Plutonium-239}

A Pu-236 tracer is added to the filter paper sample, totally dissolved, and converted to the nitrate by additions of $\mathrm{HNO}_{3}$. The plutonium is separated using an ion exchange resin, eluted off the column, and electroplated on a stainless steel disc. A solid state alpha spectrometer is utilized to count the disc, and chemical recovery is determined from the tracer peak.

\section{SILICA GEL}

Moisture is removed from the silica gel using a distillation apparatus. A portion of the distillate is mixed with a scintillation solution and counted in a liquid scintillation spectrometer. The atmospheric tritium concentration is related to the tritium concentration of the distillate through the average absolute humidity for the month during which the sample was collected.

\section{WATER}

\section{Gross Alpha (Dissolved Solids)}

After thorough agitation, a suitable aliquot is taken for analysis. The aliquot is acidified and evaporated to dryness on a hot plate after which the residue is dissolved in $1 \mathrm{~N} \mathrm{HNO}_{3}$ and transferred to a tared planchet. Gross Alpha radioactivity is then determined by counting the planchet in an internal flow proportional counter (Canberra Model 2201). 
The result is corrected for counter efficiency and self-absorption if an appreciable solid residue is obtained. For thickness 8 reater than $1 \mathrm{mg} / \mathrm{cm}^{2}$, a correction must be applied.

\section{Gross Beta (Dissolved Solids)}

This same method is used as described under Gross Alpha (Dissolved Solids). A self-absorption correction factor is applied when the sample weight exceeds $1 \mathrm{mg} / \mathrm{cm}^{2}$.

\section{Gross Alpha (Suspended Solids)}

A suitable aliquot of water is filtered through a Millipore" filter $(0.45 \mu \mathrm{m})$. The filter is counted utilizing a Canberra Model 2201 instrument. Corrections are applied for self-absorption.

\section{Gross Beta (Suspended Solids)}

The same method is used as described under Gross Alpha (Suspended Solids).

\section{Uranium-234 and -238}

The sample is digested to dryness with strong acids and redissolved in $5 \% \mathrm{HNO}_{3}$. The Uranium is separated by co-precipitation with AlPO $_{4}$. Then the precipitate is dissolved in $2 \mathrm{~N} \mathrm{Al}\left(\mathrm{NO}_{3}\right)_{3}$ and extracted into ethyl acetate. The ethyl acetate is dried and the sample transferred to a stainless steel planchet for counting in an internal flow proportional counter for its alpha activity.

\section{Plutonium-239}

The total water sample is aliquoted and acidified. $\mathrm{A} \mathrm{Pu-236}$ tracer is added to the aliquot and brought to dryness. The residue is ashed and dissolved in nitric acid. Plutonium is separated using an ion exchange resin, eluted off the column, and electroplated on a stainless steel disc. A solid state alpha spectrometer is utilized to count the disc and chemical recovery is determined from the tracer peak.

\section{Tritium}

The water sample is distilled to remove quenching materials and nonvolatile radioactive materials. Distillation is carried to dryness to ensure complete transfer of the tritium to the distillate. A portion of the distillate is mixed with scintillation solution and counted in a liquid scintillation spectrometer. Standard tritium and background samples are prepared and counted alternately to nullify errors produced by aging of the scintillation medium or instrument drift. 


\section{$\underline{\text { Radium-226 }}$}

Radium in the water sample is co-precipitated with barium sulfate. The bariumradium sulfate is separated from other alpha-emitters by dissolution with disodium EDTA, then reprecipitated as the sulfate, by addition of glacial acetic acid. The barium-radium sulfate is then transferred to a planchet for gross alpha counting.

\section{Radium-228}

The radium in the water sample is collected by co-precipitation with barium and lead sulfate, and purified by reprecipitation from EDTA solution. After a 36-hour ingrowth of actinium-228 from radium-228, the actinium-228 is carried on yttrium oxalate, purified and beta counted.

\section{SOIL}

\section{Uranium-234 and -238}

The sample is digested to dryness with strong acids and redissolved in $5 \% \mathrm{HNO}_{3}$. The Uranium is separated by co-precipitation with AlPO $_{4}$. Then the precipitate is dissolved in $2 \mathrm{~N} \mathrm{Al}\left(\mathrm{NO}_{3}\right)_{3}$ and extracted into ethyl acetate. The ethyl acetate is dried and the sample transferred to a stainless steel planchet for counting in an internal flow proportional counter for its alpha activity.

\section{Plutonium}

The soil sample is totally dissolved using a $40 \%$ solution of $\mathrm{HF}$ and a $\mathrm{Pu}-236$ tracer is added before dissolution. After fuming with $\mathrm{HF}$, the sample is brought up with $\mathrm{HNO}_{3}$. An ion exchange column is utilized to separate the plutonium which is subsequently eluted off the column and electroplated on a stainless steel counting disc. This disc is counted on a solid state alpha spectrometer and recovery is determined from the tracer peak.

\section{VEGETATION}

\section{Uranium-234 and -238}

Initially, the dry vegetation is ashed in a muffle furnace. The aliquot is digested to dryness with strong acids and redissolved in $5 \% \mathrm{HNO}_{3}$. The uranium is separated by co-precipitation with $\mathrm{AlPO}_{4}$. Then the precipitate is dissolved in $2 \mathrm{~N} \mathrm{Al}\left(\mathrm{NO}_{3}\right)_{3}$ and extracted into ethyl acetate. The ethyl acetate is dried and the sample transferred to a stainless steel planchet for counting in an internal flow proportional counter for its alpha activity. 


\section{Tritium}

The water is extracted from the vegetation sample by azeotropic distillation with benzene. The distillation is carried to dryness to ensure complete transfer of the tritium. A portion of the distillate is mixed with scintillation solution and counted utilizing a liquid scintillation spectrometer. Standard tritium and background samples are prepared and counted alternately to nullify errors produced by aging of the scintillation medium or instrument drift.

\section{QUALITY CONTROL}

\section{Radioactive and Nonradioactive}

Radioactive and nonradioactive analyses during 1988 were performed under contract by Accu-Labs Research Inc. of Wheat Ridge, Colorado. The Quality Assurance (QA) program in the Accu-Labs Radiochemistry Laboratory is designed to comply with the requirements of NRC specifications in Regulatory Guide 4.15.

The following is a summary of the Accu-Labs QA Program.

1. A major component of the QA program is the analysis of spiked and duplicate samples. The purpose of the spike analysis is to test the accuracy of the analysis and of the technician performing the analysis.

Accu-Labs routinely uses three kinds of spikes. An internal laboratory spike is prepared by the QA Department and used by the laboratory technician to determine the accuracy of the analysis prior to accepting a run. The technician knows the concentrations of these internal lab spikes. Internal lab spikes are analyzed at a frequency of $10 \%$. The QA Department also introduces blind spikes for analysis as routine samples. These samples are assigned code numbers that are indistinguishable from those on routine samples, thereby preventing preferential treatment. These spikes test the proficiency of the technician in determining accurately the composition of the spike. The QA Department prepares the blind spikes from concentrates provided by the United States Environmental Protection Agency (EPA) and Environmental Resource Associates.

In addition to the internal laboratory spikes and blind spikes, at least $10 \%$ of the samples are spiked with a known concentration of the element of interest prior to sample preparation and analysis. This procedure provides a measure of the chemical recovery of the sample preparation method as well as a verification of the analytical concentration in the sample. 
Accu-Labs technicians also analyze duplicate samples to measure the precision of the analytical method. Duplicate analysis determines the reproducibility of the method when repeated on an identical sample. The precision is in the reproducibility, not in whether the observed values are the true values. The lab technician selects, at random, duplicate samples to be analyzed. Replicate samples were run on at least $10 \%$ of all samples.

Blank samples are also run at a frequency of $10 \%$. Blanks consist of laboratory water and are used to determine any background levels of the parameters of interest.

2. Analysts, regardless of degree or experience, undergo a thorough, gradual-on-the-job training program. This is accomplished by observing an experienced chemist at work and by studying the analytical procedures. Controlled copies of official analytical procedures are provided to each analyst. Once the technician is familiar with an analytical method, a procedure review is conducted to ascertain the understanding and proficiency of the chemist. Comprehensive training records for each technician are maintained by the QA Department. Analytical results of all quality control samples are reviewed and compared by each analyst.

3. All reagents, carrier and radioactive quality control solutions which are in regular use and critical to proper results are standardized monthly, or prior to use, as appropriate.

4. All instrumentation is maintained on monthly preventive maintenance programs by the manufacturer. Each instrument is calibrated weekly with sources traceable to the National Bureau of Standards (NBS). If the calibration detects any anomaly from the routine performance of the instrument, no analysis is allowed to be performed by that instrument until it is functioning properly.

5. Quality Assurance Audits are periodically performed on each department to evaluate adherence to Accu-Labs QA plan. Results of these audits are provided to the area supervisors. Any observation reports or corrective action reports must be reviewed and a response made to the quality control of ficer.

6. Interlaboratory comparisons are conducted with the U.S. Public Health Service, Environmental Protection Agency, U.S. Department of Energy (EML Quality Assurance Program Table A-II), Department of the Navy, and the American Industrial Hygiene Association. In addition, this laboratory is a member with good standing in the American Association for Laboratory Accreditation. 
Table A-II. Laboratory Listing from EML Quality

Assurance Program. (EML-518)

\begin{tabular}{|c|c|c|c|c|c|c|c|c|}
\hline \multirow[b]{2}{*}{ Date } & \multirow[b]{2}{*}{ Type } & \multirow[b]{2}{*}{ Lab } & \multirow[b]{2}{*}{ Isotope } & \multirow[b]{2}{*}{$\underline{\text { Ser }}$} & \multicolumn{2}{|c|}{ Reported } & \multirow[b]{2}{*}{ EML Value } & \multirow[b]{2}{*}{$\begin{array}{l}\text { Ratio } \\
\text { Ro/EML }\end{array}$} \\
\hline & & & & & Value & $\begin{array}{c}x \\
\text { Error }\end{array}$ & & \\
\hline $3 / 88$ & Air & $P A^{*}$ & PU 239 & 1 & $0.280 \times 10^{1}$ & 46 & $0.252 \times 10^{1}$ & $1.11 \pm 0.52$ \\
\hline $3 / 88$ & Air & $P A$ & U 234 & 1 & $0.260 \times 10^{1}$ & 30 & $0.252 \times 10^{1}$ & $1.03 \pm 0.32$ \\
\hline $3 / 88$ & Air & $P A$ & U 238 & 1 & $0.230 \times 10^{1}$ & .47 & $0.253 \times 10^{1}$ & $0.91 \pm 0.44$ \\
\hline $3 / 88$ & Soil & $P A$ & PU 239 & 1 & $0.700 \times 10^{-1}$ & 28 & $0.410 \times 10^{-1}$ & $1.71 \pm 3.78$ \\
\hline $3 / 88$ & Soil & $P A$ & U 234 & 1 & 0.500 & 40 & 0.670 & $0.75 \pm 0.30$ \\
\hline $3 / 88$ & Soil & $P A$ & U 238 & 1 & 0.400 & 50 & 0.690 & $0.58 \div 0.29$ \\
\hline $3 / 88$ & Vegetation & $P A$ & PU 239 & 1 & $0.700 \times 10^{-1}$ & 42 & $0.450 \times 10^{-1}$ & $1.56 \pm 0.67$ \\
\hline $3 / 88$ & Vegetation & $P A$ & U 234 & 1 & $0.300 \times 10^{-1}$ & 66 & $0.360 \times 10^{-1}$ & $0.83 \pm 0.59$ \\
\hline $3 / 88$ & Vegetation & $P A$ & U 238 & 1 & $0.300 \times 10^{-1}$ & 66 & $0.360 \times 10^{-1}$ & $0.83 \pm 0.59$ \\
\hline $3 / 88$ & Water & $P A$ & H3 & 1 & $0.205 \times 10^{2}$ & 2 & $0.207 \times 10^{2}$ & $0.99 \neq 0.05$ \\
\hline $3 / 88$ & Water & $P A$ & PU 239 & 1 & $0.210 \times 10^{-1}$ & 23 & $0.243 \times 10^{-1}$ & $0.86 \pm 0.22$ \\
\hline $3 / 88$ & Water & $P A$ & U 234 & 1 & $0.300 \times 10^{-2}$ & 66 & $0.425 \times 10^{-2}$ & $0.71 \div 0.47$ \\
\hline $3 / 88$ & Water & $P A$ & U 238 & 1 & $0.400 \times 10^{-2}$ & 50 & $0.425 \times 10^{-2}$ & $0.94 \div 0.48$ \\
\hline $9 / 88$ & Air & $P A^{*}$ & PU 239 & 1 & $0.170 \times 10^{1}$ & 64 & $0.109 \times 10^{1}$ & $1.56 \pm 1.01$ \\
\hline $9 / 88$ & Air & $P A$ & U 234 & 1 & $0.160 \times 10^{1}$ & 118 & $0.240 \times 10^{1}$ & $0.67 \neq 0.79$ \\
\hline $9 / 88$ & Air & $P A$ & U 238 & 1 & $0.240 \times 10^{1}$ & 91 & $0.239 \times 10^{1}$ & $1.00 \pm 0.92$ \\
\hline $9 / 88$ & Soil & $P A$ & PU 239 & 1 & 0.450 & 35 & 0.380 & $1.18 \pm 0.42$ \\
\hline $9 / 88$ & Soil & $P A$ & U 234 & 1 & 0.740 & 27 & 0.790 & $0.94 \div 0.25$ \\
\hline $9 / 88$ & Soil & $P A$ & U 238 & 1 & 0.810 & 25 & 0.790 & $1.03 \pm 0.27$ \\
\hline $9 / 88$ & Vegetation & $P A$ & PU 239 & 1 & $0.280 \times 10^{-1}$ & 117 & $0.210 \times 10^{-1}$ & $1.33 \pm 1.58$ \\
\hline $9 / 88$ & Vegetation & $P A$ & U 234 & 1 & $0.210 \times 10^{-1}$ & 252 & $0.230 \times 10^{-1}$ & $0.91 \pm 2.31$ \\
\hline $9 / 88$ & Vegetation & PA & U 238 & 1 & $0.260 \times 10^{-1}$ & 249 & $0.180 \times 10^{-1}$ & $1.44 \pm 3.61$ \\
\hline $9 / 88$ & Water & PA & H 3 & 1 & $0.102 \times 10^{-2}$ & 3 & $0.106 \times 10^{-2}$ & $0.96 \pm 0.06$ \\
\hline $9 / 88$ & Water & PA & PU 239 & 1 & $0.480 \times 10^{-2}$ & 43 & $0.540 \times 10^{-2}$ & $0.89 \pm 0.40$ \\
\hline $9 / 88$ & Water & PA & U 234 & 1 & $0.460 \times 10^{-2}$ & 58 & $0.410 \times 10^{-2}$ & $1.12 \pm 0.66$ \\
\hline $9 / 88$ & Water & $P A$ & U 238 & 1 & $0.450 \times 10^{-2}$ & 55 & $0.420 \times 10^{-2}$ & $1.07 \pm 0.60$ \\
\hline
\end{tabular}

*Pantex via Accu-Labs

\section{BOD. COD, and Fecal Coliform}

BOD and COD were run in our own Environmental Health Laboratory, using procedures in Standard Methods, 15th Edition. An Alpha-Trol ${ }^{\text {m }}$ standard was run with each set of samples.

Fecal coliform samples were analyzed by the Amarillo Bi-City County Health Department of the City of Amarillo. This is a state-supported state-certified laboratory. 


\begin{tabular}{llr}
\hline Table A-III. & $\begin{array}{l}\text { Radioactivity Concentration Guides (RCG) } \\
\text { for Controlled Areas (15) }\end{array}$ \\
& $\begin{array}{c}\text { Air } \\
(U \mathrm{Ci} / \mathrm{ml})\end{array}$ & $\begin{array}{c}\text { Water } \\
(\mathrm{UCi} / \mathrm{ml})\end{array}$ \\
& $2 \times 10^{-12}$ & $1 \times 10^{-4}$ \\
Plutonium-239 & $5 \times 10^{-6}$ & $1 \times 10^{-1}$ \\
Tritium & $2 \times 10^{-11}$ & $4 \times 10^{-6}$ \\
Uranium-234 & $7 \times 10^{-11}$ & $2 \times 10^{-5}$ \\
Uranium-238 & $3 \times 10^{-11}$ & $4 \times 10^{-7}$ \\
Radium-226 & $7 \times 10^{-11}$ & $8 \times 10^{-7}$ \\
Radium-228 & & 8
\end{tabular}

\begin{tabular}{|c|c|c|}
\hline \multicolumn{3}{|c|}{$\begin{array}{c}\text { Table A-IV. Derived Concentration Guides (DCG) } \\
\text { for Members of Public(22) }\end{array}$} \\
\hline & $\begin{array}{c}\text { Air } \\
(u C i / m I) \\
\end{array}$ & $\begin{array}{c}\text { Water } \\
(u C i / m])\end{array}$ \\
\hline $\begin{array}{l}\text { Plutonium-239 } \\
\text { Tritium } \\
\text { Uranium-234 } \\
\text { Uranium-238 } \\
\text { Radium-226 } \\
\text { Radium-228 }\end{array}$ & $\begin{array}{l}2 \times 10^{-14} \\
1 \times 10^{-7} \\
9 \times 10^{-14} \\
1 \times 10^{-13} \\
1 \times 10^{-12} \\
3 \times 10^{-12}\end{array}$ & $\begin{array}{l}3 \times 10^{-8} \\
2 \times 10^{-3} \\
5 \times 10^{-7} \\
6 \times 10^{-7} \\
1 \times 10^{-7} \\
1 \times 10^{-7}\end{array}$ \\
\hline
\end{tabular}

\begin{tabular}{|c|c|c|c|}
\hline \multirow{5}{*}{$\begin{array}{l}\text { Fossile Fuel } \\
\text { Steam Generator }\end{array}$} & \multicolumn{3}{|c|}{$\begin{array}{l}\text { Performance Standards for Stationary Sources } \\
\text { Reference }\end{array}$} \\
\hline & \multirow[t]{4}{*}{40 CFR 60.40} & Particulate: & $0.10 \mathrm{lb} / 10^{6} \mathrm{BTU}$ \\
\hline & & Opacity: & $20 \%$ \\
\hline & & $\mathrm{SO}_{2}:$ & $\begin{array}{l}0.80 \mathrm{lb} / 10^{6} \text { BTU } \\
\text { (liquid Fuel) }\end{array}$ \\
\hline & & $\mathrm{NO}_{2}:$ & $\begin{array}{l}0.201 \mathrm{~b} / 10^{6} \text { BTU } \\
\text { (gas fue } 1) \\
0.301 \mathrm{~b} / 10^{6} \text { BTU } \\
\text { (i iquid Fuel) }\end{array}$ \\
\hline
\end{tabular}


Table A-VI. Water Sample Locations

\begin{tabular}{ll} 
Sample No. & \multicolumn{1}{c}{ Description of Location } \\
OW-WR-01 & Pantex Playa Lake \\
OW-WR-02 & Water Well 17 \\
OW-WR-06 & Water Well 16 \\
OW-WR-08 & East Bank of North Retention Basin Playa \\
OW-WR-09 & Main Drainage Ditch (East Side of Zone 12) \\
OW-WR-13 & Bldg. 12-43 HE Filter Building Effluent \\
OW-WR-15 & Bldg. 11-44 HE Filter Building Effluent \\
OW-WR-16 & Water Well I20 \\
OW-WR-17 & Sewage Discharge \\
OW-WR-18 & Water Well 16 \\
OW-WR-19 & North Retention Basin Playa Monitor Well 1 \\
OW-WR-20 & Zone 12 Monitor Well \\
OW-WR-23 & Orinking Water from Bldg. 12-2 \\
OW-WR-24 & South Playa, Due South of Zone 12 \\
OW-WR-25 & Drainage from 12-64 to South Playa \\
OW-WR-26 & Bldg. 12-73 Decontamination Building Effluent \\
OW-WR-27 & West Playa near Sanitary Landfill \\
OW-WR-28 & Drinking Water from 12-6 Cafeteria \\
OW-WR-30 & Spiked Sample \\
OW-WR-31 & Effluent Pond, Zone 11 East \\
OW-WR-33 & Well at Bushland Agricultural Experimental Station \\
OW-WR-36 & Burning Ground Playa Lake \\
OW-WR-37 & Private Water Well West of Plant \\
OW-WR-38 & Northeast Monitor Well \\
OW-WR-39 & Northwest Monitor Well \\
OW-WR-40 & Southwest Monitor Well \\
OW-WR-43 & Orinking Water from Firing Site (FS-1) \\
OW-WR-44 &
\end{tabular}




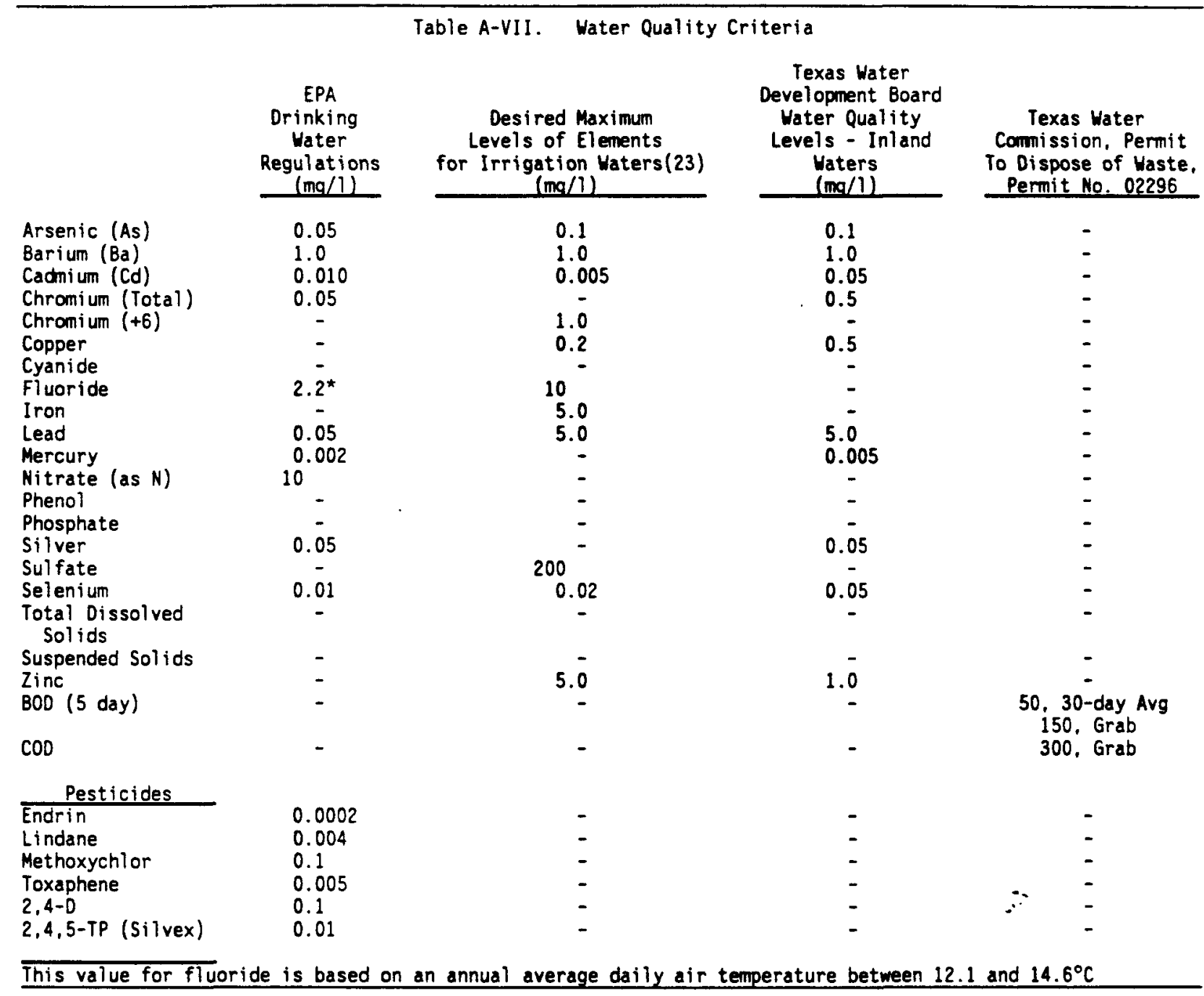

